\title{
Protein dynamics in the nucleus: Implications for gene expression
}

\section{PhD Thesis}

in partial fulfilment of the requirements for the degree "Doctor of Philosophy (PhD)" in the Molecular Biology Program at the Georg August University Göttingen,

Faculty of Biology

submitted by

\section{Gabriella Ficz}

born in

\section{Miercurea-Ciuc, Romania}


The experimental part of this thesis was performed at the

\section{Max-Planck-Institute for biophysical Chemistry Department of Molecular Biology Göttingen, Germany}

Thesis committee members:

Dr. Donna Arndt-Jovin

Prof. Dr. Herbert Jäckle

Prof. Dr. Hans-Joachim Fritz

Date of submission of the PhD Thesis: June $15^{\text {th }}, 2005$ 
Mamei mele 


\section{TABLE OF CONTENTS}

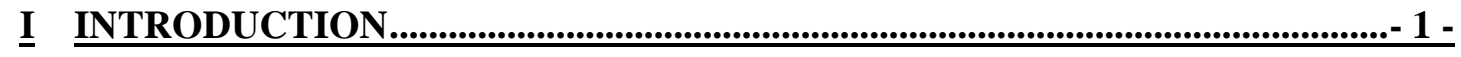

I.1 NUCLEAR ARCHITECTURE AND GENE EXPRESSION .............................................. - 1 -

I.2 HOMEOTIC GENES AND DEVELOPMENT OF MULTICELLULAR ORGANISMS ............ - 4 -

I.3 THE POLYCOMB GROUP PROTEINS ....................................................................... - 6 -

I.4 PhOtOBleACHING MicRoscopy AND MOBILITY OF MOLECULES INSIDE CELLS - 12 -

I.5 COMPUTER SIMULATIONS - A MEANS TO OBTAIN A BETTER INSIGHT INTO CELLULAR

PROCESSES...................................................................................................................... 14 -

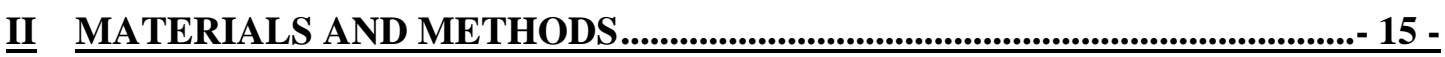

II.1 MOLECULAR BIOLOGY MATERIALS AND METHODS................................................ - 15 -

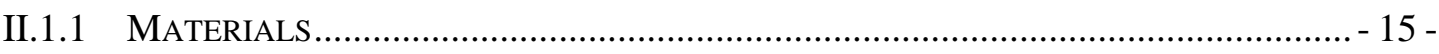

II.1.1.1 Chemicals and enzymes ........................................................................ 15 -

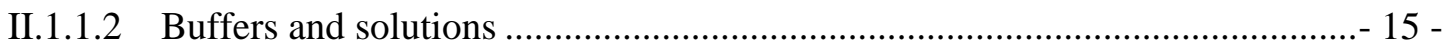

II.1.1.3 Bacterial hosts, media and culture plates .................................................... 16 -

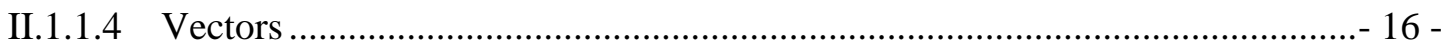

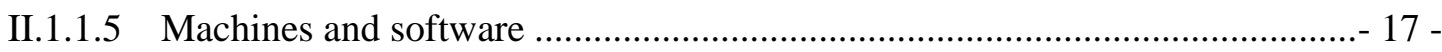

II.1.2 MOLECULAR BIOLOGY AND BIOCHEMISTRY METHODS ....................................... - 17 -

II.1.2.1 Plasmid DNA purification from E. coli and determination of DNA

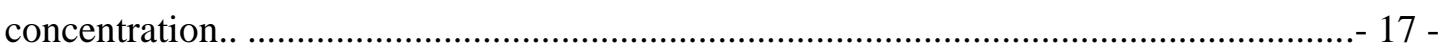

II.1.2.2 Agarose gel electrophoresis and DNA elution from gels .............................. 17 -

II.1.2.3 Polymerase chain reaction (PCR) ................................................................. 18 -

II.1.2.4 Primer design and cloning strategy ............................................................ 19 -

II.1.2.5 Restriction enzyme digestion of DNA and ligation reactions ........................ 20 -

II.1.2.6 Transformation of competent bacteria .......................................................... 21 -

II.1.2.7 Total protein extraction from Drosophila tissues ............................................ 21 -

II.1.2.8 Polyacrylamide gel electrophoresis (SDS-PAGE).......................................... 23 -

II.1.2.9 Immunoblotting and proteins quantification on western blots ........................-24 -

II.1.2.10 TUNEL assay for apoptosis detection in Drosophila tissues ......................... 25 -

II.2 DROSOPHILA GENETICS METHODS ................................................................ - 26 - 
II.2.1 GENERATION OF TRANSGENIC FLY LINES …............................................... - 26 -

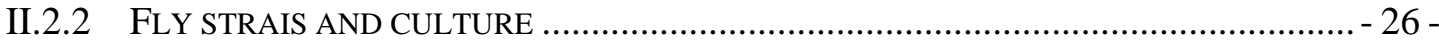

II.2.3 ATTEMPT TO RESCUE OF APOPTOSIS USING A P53 MUTANT LINE ........................... - 28 -

II.2.4 RESCUE OF PH NULL MUTATION PHENOTYPE BY PHGFP ..................................... - 29 -

II.3 BIOPHYSICAL MATERIALS AND METHODS............................................................ - 30 -

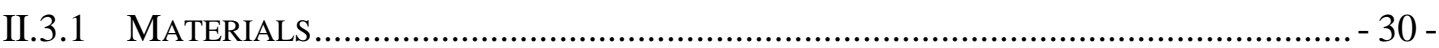

II.3.1.1 Phase contrast microscope ................................................................... 30 -

II.3.1.1.1 Mounting of specimens for microscopy and imaging................................. 30 -

II.3.1.2 Confocal scanning microscope …............................................................. 31 -

II.3.1.2.1 Mounting of specimens for microscopy and imaging.................................. 31 -

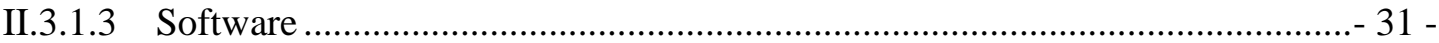

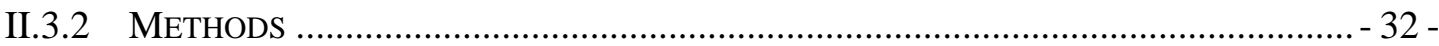

II.3.2.1 Photobleaching methods .............................................................................. 32 -

II.3.2.1.1 FRAP (fluorescence recovery after photobleaching)...................................- 33 -

II.3.2.1.2 iFRAP (inverse FRAP) ......................................................................... 34 -

II.3.2.1.3 FLIP (fluorescence loss in photobleaching) .............................................. 34 -

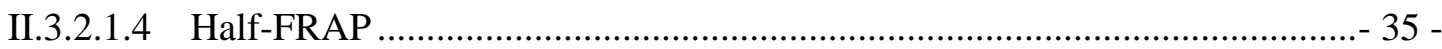

II.3.2.2 Image processing and fitting algorithms ....................................................... 35 -

II.3.2.3 Simulation methods: differential equations for the binding and dissociation of fluorescent and non-fluorescent molecules to fixed binding sites................................. 39 -

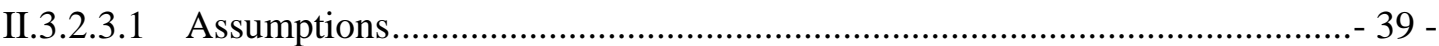

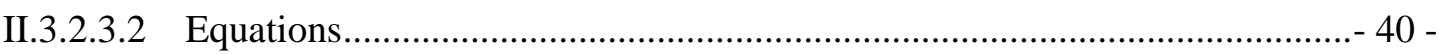

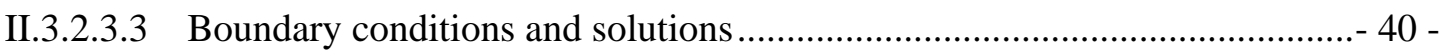

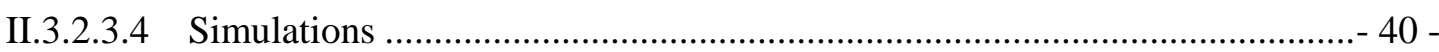

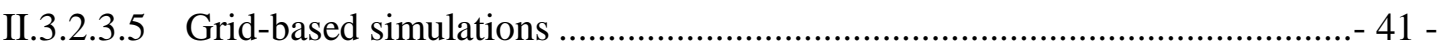

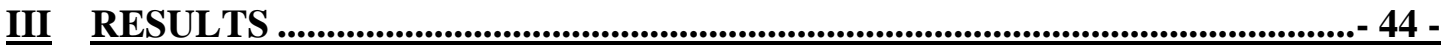

III.1 NUCLEAR DISTRIBUTION AND DYNAMICS OF PCG PROTEIN COMPLEXES IN

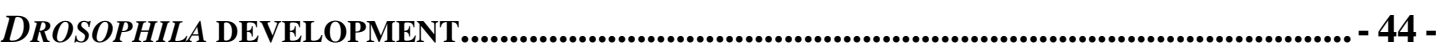

III.1.1 EXPRESSION PATTERN OF PCGFP AND PHGFP IN VARIOUS DEVELOPMENTAL

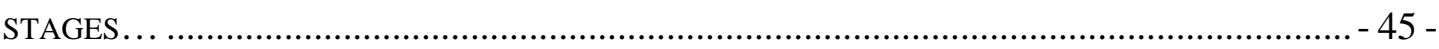


III.1.2 EXTENSIVE MOBILITY OF PHGFP AND PCGFP COMPLEXES IN LIVE GASTRULATING EMBRYOS AND WHOLE MOUNT IMAGINAL DISCS $-48-$

III.2 MEASURING DIFFUSION CONSTANTS OF NUCLEAR PROTEINS ............................ - 52 -

III.2.1 DIFFUSION OF PCGFP AND PHGFP IN THE DROSOPHILA NUCLEI IN EMBRYOS AND LARVAL TISSUES $-53-$

III.2.2 SIMULATION OF DIFFUSION PROCESSES WITH VARIOUS BOUNDARY CONDITIONS - 55 III.3 PHOTOBLEACHING IN LIVING DROSOPHILA EMBRYOS AND TISSUES: POSSIBILITIES AND LIMITATIONS. $-57-$

III.3.1 CLASSICAL PHOTOBLEACHING TECHNIQUES (FRAP, IFRAP, FLIP) ARE NOT APPLICABLE TO ANALYZE DROSOPHILA PCG COMPLEXES IN EMBRYOS AND LARVAL TISSUES. $-58-$

III.3.2 SIMULATION OF THE OUT-OF-FOCUS BLEACHING $-60-$

III.3.3 3D-IFRAP: NEW ADAPTED PHOTOBLEACHING TECHNIQUE TO DETERMINE DISSOCIATION CONSTANTS OF PCG PROTEINS IN DROSOPHILA $-62-$

\section{III.4 DiSSOCIATION RATE CONSTANTS OF PCG COMPLEXES IN NUCLEI OF EMBRYOS} AND IMAGINAL DISCS.

III.5 MODEL OF THE BINDING REACTIONS OF PROTEINS TO LOCALIZED CHROMATIN

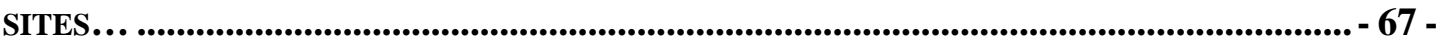

III.6 SIMULATION OF BINDING REACTIONS TO LOCALIZED CHROMATIN SITES.......... - 69 -

III.6.1 PARAMETERS OF A SIMULATION EXPERIMENT. $-70-$

III.6.2 FRAP RECOVERY TIME DEPENDENCE ON THE AMOUNT OF PROTEIN BOUND TO THE TARGET SITE -72 -

III.6.3 THE “EATING UP” EFFECT IN FRAP $-74-$

III.7 Distribution, DYNAMICS AND DisSOciation RATE CONSTANTS OF PCG PROTEINS ON DROSOPHILA POLYTENE CHROMOSOMES................................................... - 76 -

III.7.1 PCG COMPLEXES HAVE DIFFERENT RESIDENCE TIMES ON INDIVIDUAL BANDS IN

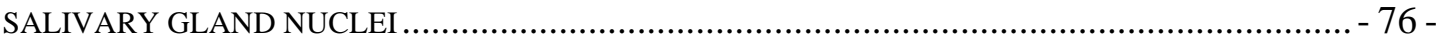

III.8 RESCUE OF THE PH NULL PHENOTYPE WITH PHGFP ...................................... - 82 III.9 OVEREXPRESSION OF PHGFP INDUCES APOPTOSIS IN ALL DEVELOPMENTAL

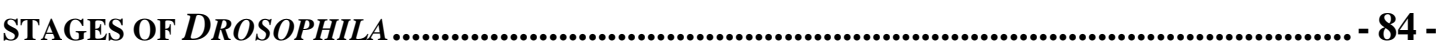

III.10 FUTURE EXTENSIONS OF THE TECHNIQUE TO OTHER PCG COMPLEXES......... - 86 -

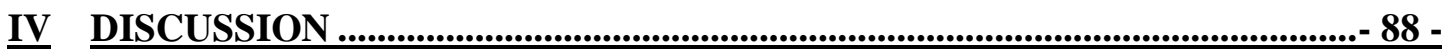


IV.1 IN VIVO EXPERIMENTS TO INVESTIGATE THE STABILITY OF PCG REPRESSION

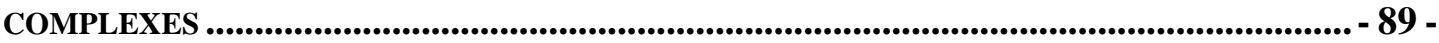

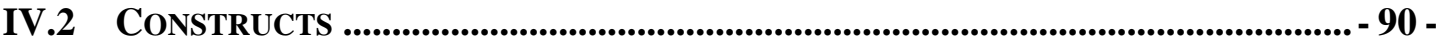

IV.3 PHOTOBLEACHING MICROSCOPY IN WHOLE ORGANISMS.................................... - 90 -

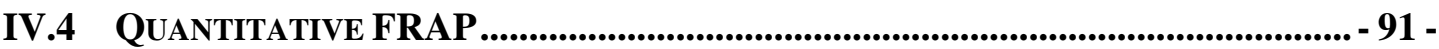

IV.5 3D-IFRAP: A NEW PHOTOBLEACHING TECHNIQUE ......................................... - 92 -

IV.6 THE INFLUENCE OF DIFFUSION AND THE AMOUNT OF UNBOUND PROTEIN ON FRAP DATA; DETERMINATION OF RATE CONSTANTS ................................................................... - 92 -

IV.6.1 DIFFUSION PROCESSES AND BINDING OF PCG PROTEINS TO PRES ....................... - 92 -

IV.6.2 DETERMINATION OF DISSOCIATION RATE CONSTANTS FROM FRAP DATA.......... - 93 -

IV.6.3 DETERMINATION OF PSEUDO-ASSOCIATION RATE CONSTANTS FROM THE

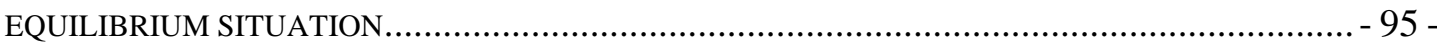

IV.7 SIMULATION OF DIFFUSION AND BINDING REACTIONS .......................................... - 96 -

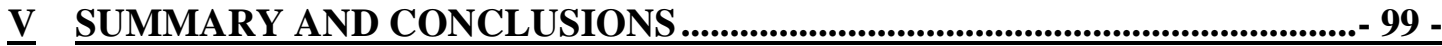

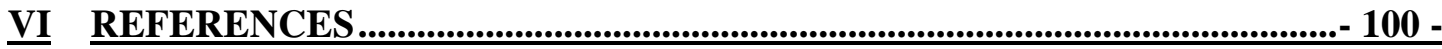

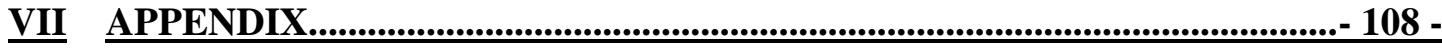

VII.1 GRID BASED DIFFUSION AND BINDING SIMULATION......................................... - 108 -

VII.2 ESTIMATION OF GAMMA FACTORS …................................................................ - 111 -

VII.3 SIMULATION OF BINDING KINETICS WITH GIVEN CONCENTRATIONS.............. - 112 -

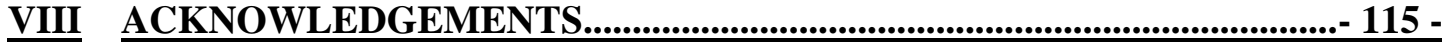

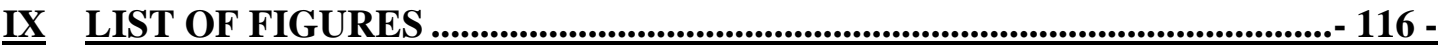

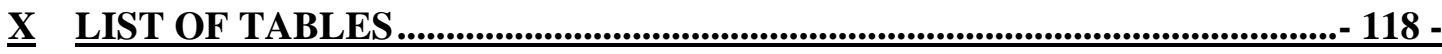




\section{LIST OF ABBREVIATIONS}

\begin{tabular}{|c|c|}
\hline $2 \mathrm{~N}$ & diploid \\
\hline 3D & 3-dimensional \\
\hline abdA & abdominal A \\
\hline AbdB & abdominal B \\
\hline ADI & Alternating Directions Implicit in Time \\
\hline ANT-C & Antennapedia Complex \\
\hline Antp & Antennapedia \\
\hline AOTF & Acusto Optical. Tunable Filter \\
\hline BX-C & Bithorax Complex \\
\hline ChIP & Chromatin Immunoprecipitation \\
\hline Dfd & Deformed \\
\hline DNA & Deoxyribonucleic Acid \\
\hline $\mathrm{E}(\mathrm{Z})$ & Enhancer of Zeste \\
\hline ESC & Extra sex combs \\
\hline FCS & Fluorescence Correlation Spectroscopy \\
\hline FLIP & Fluorescent Loss in Photobleaching \\
\hline FRAP & Fluorescence Recovery After Photobleaching \\
\hline GFP & Green Fluorescent Protein \\
\hline Hsc4 & Heat shock protein cognate 4 \\
\hline hsp26 & heat shock protein 26 \\
\hline iFRAP & inverse FRAP \\
\hline lab & labial \\
\hline mRNA & messenger RNA \\
\hline $\mathrm{Pb}$ & Proboscipedia \\
\hline Pc & Polycomb \\
\hline PCC & Polycomb core complex \\
\hline PcG & Polycomb Group \\
\hline PCR & Polymerase Chain Reaction \\
\hline $\mathrm{Ph}$ & Polyhomeotic \\
\hline Pho & Pleiohomeotic \\
\hline PRC1 & Polycomb Repressor Complex 1 \\
\hline PRC2 & Polycomb Repressor Complex 2 \\
\hline PRE & Polycomb Response Elements \\
\hline RFP & Red Flourescent Protein \\
\hline RNA & Ribonucleic Acid \\
\hline ROI & Region of Interest \\
\hline rRNA & ribosomal RNA \\
\hline Scr & Sex combs reduced \\
\hline SDS & Sodium Dodecyl Sulphate \\
\hline $\mathrm{Su}(\mathrm{Z})$ & Supressor of Zeste \\
\hline
\end{tabular}




\begin{tabular}{|c|c|}
\hline TdT & Terminal deoxynucleotidyl Transferase \\
\hline $\operatorname{trx} G$ & Trithorax Group \\
\hline TUNEL & $\begin{array}{l}\text { Terminal deoxynucleotidyl transferase-mediated d } \underline{\mathbf{U}} \text { TP } \underline{\text { Nick }} \text { End- } \\
\underline{\text { L}} \text { abeling }\end{array}$ \\
\hline Ubx & Ultrabithorax \\
\hline VFP & Visible Fluorescent Proteins \\
\hline wt & wild type \\
\hline Z & Zeste \\
\hline
\end{tabular}




\section{ABSTRACT}

Fluorescence recovery after photobleaching (FRAP) microscopy was used to determine the kinetic properties of Polycomb group proteins in whole living Drosophila organisms (embryos) and tissues (wing imaginal discs and salivary glands). These are the first photobleaching experiments performed in whole embryos and tissues.

Polycomb group (PcG) genes are essential genes in higher eukaryotes responsible for the maintenance of the spatially distinct repression of developmentally important regulators such as the homeotic genes. Their absence, as well as overexpression, causes transformations in the axial organization of the body. Although protein complexes have been isolated in vitro, little is known about their stability or exact mechanism of repression in vivo.

I determined the translational diffusion coefficients of PcG proteins, dissociation constants and residence times for complexes in vivo at different developmental stages. In polytene nuclei the rate constants suggest heterogeneity of the complexes. Computer simulations with new models for spatially distributed protein complexes were performed in systems showing both diffusion and binding equilibria and the results compared with the experimental data. I was able to determine forward and reverse rate constants for complex formation. Complexes exchanged within a period of one to ten minutes, more than an order of magnitude faster than the cell cycle time, ruling out models of repression in which access of transcription activators to the chromatin is limited and demonstrating that longterm repression primarily reflects mass-action chemical equilibria.

With the help of computer programs built to simulate diffusion in boundary conditions and binding kinetics of proteins to localized binding sites in the genome I describe in detail the observed biophysical processes underlying FRAP and offer guidance for a better setup, optimization and interpretation of photobleaching experiments. 


\section{INTRODUCTION}

\section{I.1 Nuclear architecture and gene expression}

A fundamental question in biology is how a concert of gene expression is orchestrated in a highly packed environment as in eukaryotic nuclei. Gene expression is a continuous process carried out by highly specialized machineries responsible for chromatin remodeling, transcription, RNA processing, RNA export and translation in the cytoplasm (Misteli, 2001; Orphanides and Reinberg, 2002). The process of transcription itself takes place in the nucleus that is a complex organelle both in organization and in function. Contemporary studies support the concept that the three dimensional organization of the nucleus has a high impact on the functional diversity of the cells (Misteli, 2005), i.e. each gene locus might be characterized by specific nuclear environments. Organization of chromatin in the nucleus is non-random as chromosomes tend to occupy specific regions inside nuclei relative to the nuclear periphery and relative to each other (Parada and Misteli, 2002). The presence of morphologically higher order chromatin domains like euchromatin (loosely packed) and heterochromatin (tightly packed) divides the genome into transcriptionally active and inactive territories, respectively. This view proved to be oversimplistic since a genome wide study by Bickmore and colleagues on the relationship chromatin structure $\rightarrow$ gene activity found instead a correlation gene-rich region: open chromatin structure, gene-poor region: condensed domain, regardless of the activity status of the gene (Gilbert et al., 2004). Chromosomes exist in the nucleus in the form of chromosome territories (Cremer and Cremer, 2001; Parada and Misteli, 2002). The location of a gene within a chromosome territory seems to influence its access to the machinery responsible for specific nuclear functions, such as transcription and splicing. Chromosome territories are structures permeated by nucleoplasmic channels of various sizes that create large surface areas accessible for regulatory factors to sequences buried within the chromosome territories (Cremer and Cremer, 2001). Various membrane-less nuclear compartments like the nucleolus and distinct nuclear bodies separate functional domains for highly specialized processes like rRNA biogenesis processing, assembly of ribosomal subunits, pre-mRNA splicing, snRNP biogenesis and assembly etc (Fig.1). The cartoon in Fig. 1 represents a static view of the nucleus. Contrary, these domains have been 
shown in a series of in vivo experiments to be dynamic structures and, in addition, rapid protein exchange occurs between many of the domains and the nucleoplasm (Misteli, 2001). But there is still a big step from the dynamic nucleus to a multicellular organism with spatial and temporal organization. This implies accomplishment of a complex genetic program initiated at a single cell level (the fertilized egg or zygote) and finalized in multicellular environment in the embryo and adult.

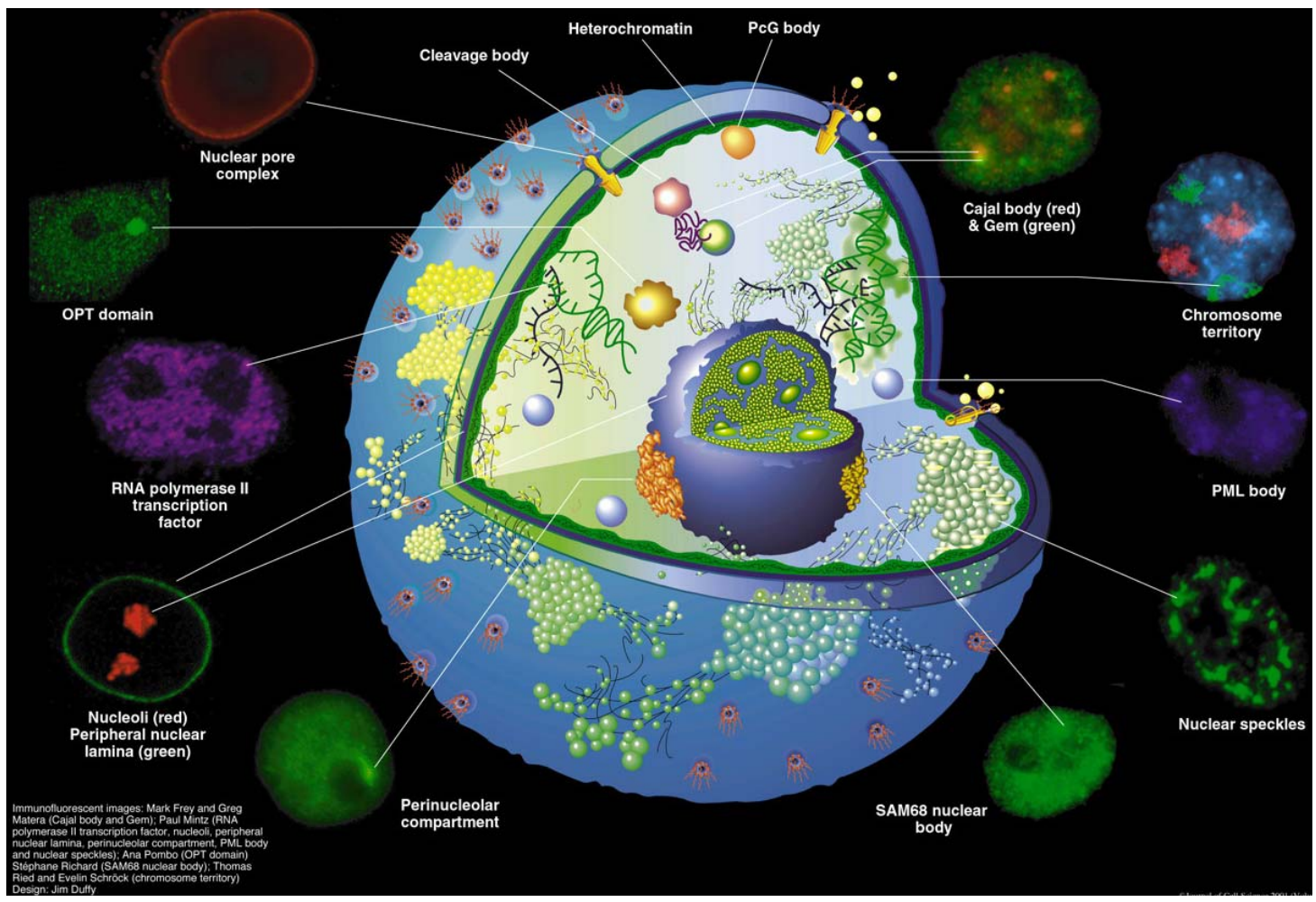

Figure 1 The cell nucleus: organization of the nucleus into specialized domains. The nuclear content is delimited by the nuclear envelope with the nuclear pores serving as transit material between the nucleus and the cytoplasm. The peripheral nuclear lamina regulates the nuclear envelope structure and anchors the interphase chromatin at the nuclear periphery; heterochromatin (inactive chromatin) is associated with the nuclear lamina; PcG bodies, containing polycomb group proteins, are involved in silencing; pre-mRNA splicing factors are localized in nuclear speckles as well as diffusely distributed throughout the nucleoplasm. Transcription sites are observed throughout the nucleoplasm but they have also been shown to be concentrated in one to three compartments termed OPT (Oct1/PTF/transcription) domains; snRNP biogenesis takes place in 1-10 Cajal bodies whereas their Gemini (Gems) might be responsible for snRNP assembly and maturation; Cleavage bodies might be involved in the cleavage and polyadenylation steps of pre-mRNA processing. The nucleolus is the site of rRNA synthesis, rRNA processing and assembly of ribosomal subunits; the associated 
perinucleolar compartment and the SAM68 nuclear body might play a role in RNA metabolism. PML bodies have been suggested to play a role in aspects of transcriptional regulation and appear to be targets of viral infection (adapted from Spector DL, 2001)

Differential gene expression is the platform that allows formation of cellular diversity necessary for generating multicellular organisms and the physiological relevant substrate in this case is the chromatin (Felsenfeld and Groudine, 2003). The chromatin is a DNA-histone protein complex made up of repeating organizational units called nucleosomes. In a nucleosome a 146 bp double stranded DNA wraps around a central core of eight histone protein molecules (an octamer made of histones H2A, H2B, H3 and H4). Histone $\mathrm{H} 1$ binds to the linker DNA (between two nucleosomes) and fastens the DNA to the nucleosome core. Until recently, our understanding of genetic diseases has been very much related to alteration of the DNA sequence.

In the last decades, chemical modifications of the DNA (methylation) and of histones (methylation, acetylation, phosophorylation, ubiquitination etc.) have been a subject of major interest due to their implication in fundamental cellular and developmental processes (i.e. transcription activation of repression, differentiation, etc.) and found to play a role in many human diseases (Feinberg et al., 2002).

Since epigenetic alterations in human tumours have been discovered in 1983 (Feinberg and Vogelstein, 1983), hundreds of labs have examined their role both in activation of tumour promoter genes as well as silencing of tumour suppressor genes. The combinatorial nature of histone modifications is hypothesized to define a "histone code" that considerably extends the information potential of the genetic code, giving rise to epigenetic information. Moreover, most core histones consist of several nonallelic variants that can mark specific loci and could play an important role in establishment and maintenance of epigenetic memory. Unravelling the "histone code" is therefore necessary and very challenging since epigenetic mechanisms lie at the very heart of our understanding of stem cell therapy, animal cloning, complex traits and aging.

Model organisms have provided key insights into cellular processes that have been recognized in higher eukaryotes such as humans. I used Drosophila melanogaster as a model organism since it is a well known developmental model system, is easy to handle, the life cycle is short, is subject to easy genetic manipulation, the genome is sequenced and there is an enormous collection of mutants that enable complex experimentation in a living organism. 
As a result, most of what we know about the molecular basis of animal development has come from studies of model systems such as Drosophila.

\section{I.2 Homeotic genes and development of multicellular organisms}

Development of an organism from a single cell to an adult is a result of key mechanisms that occur sequentially or simultaneously: unequal distribution of cytoplasmic materials (polarity); sequential and hierarchical expression of different groups of developmental genes (regulatory cascades); definition of functional characteristics of the body by homeotic genes; induction of organ development by morphogens, and programmed cell death or apoptosis. The knowledge we have about early Drosophila development is the result of the pioneering work of Ed Lewis, Christiane Nüsslein-Volhard and Eric Wieschaus. Their approach was to apply saturation mutagenesis to the fly to try and identify every gene involved in early anterior-posterior pattern formation. They looked at larval cuticle patterns from thousands of fly mutations and were spectacularly successful in identifying the major classes of genes involved in generating the segmental body plan of the fly. In short, following fertilization, a small number of maternally inherited determinants trigger a cascade of spatially and temporally regulated transcriptional events that will lead to an embryo divided in smaller and smaller domains (Fig. 2A). Maternal genes define the anterior-posterior axis of the embryo that will be subdivided into broad areas by the gap genes; the pair rule genes define segments that divide the embryo into units of two segments and the segment polarity genes will determine anterior-posterior organization of individual segments. The resulting expression of these various genes and their gene products in the 14 segments will be represented by a particular combination that will lead to activation of homeotic genes.

Homeotic genes define the identity of a particular segment (Lewis, 1978; SanchezHerrero et al., 1985). They are expressed differentially along the anterior-posterior and dorsal-ventral axis of the embryo, and it is this differential expression that directs the parts of the embryo to follow different developmental pathways. 
A

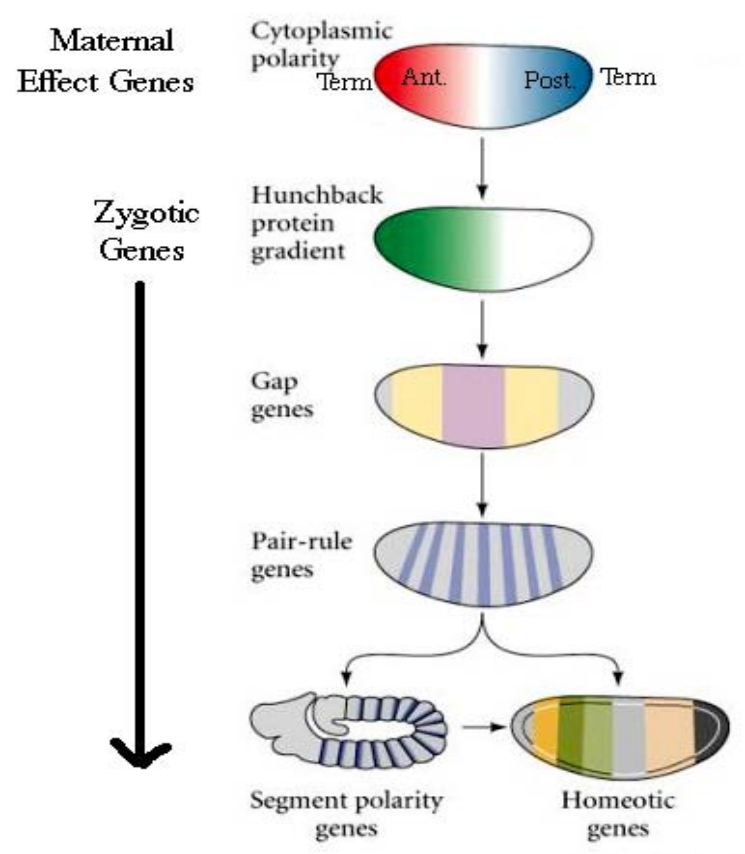

B

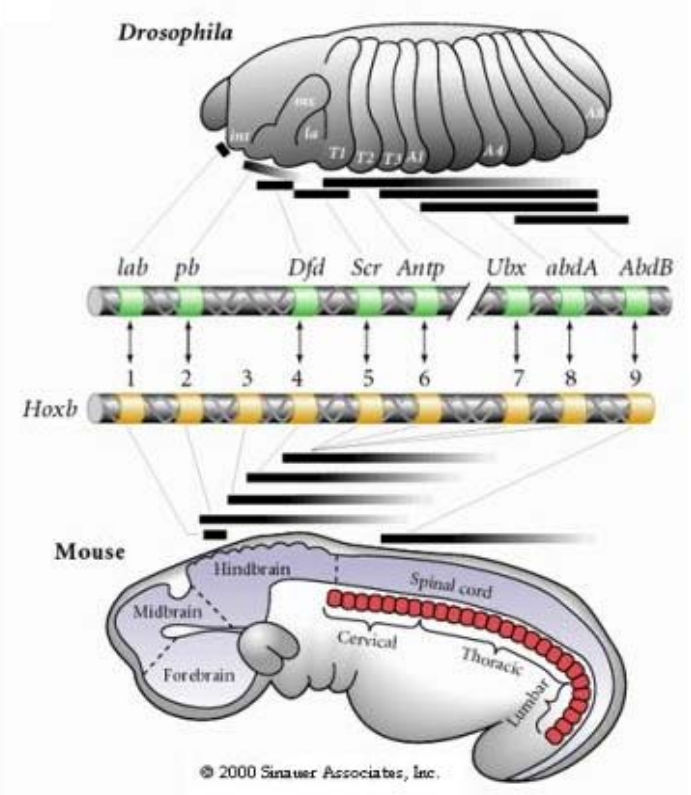

Figure 2. Pattern formation in Drosophila. A) Proteins like bicoid and nanos (maternal effect-genes) form a morphogenetic gradient along the A-P axis; they will regulate the expression pattern of the gap genes. The gap genes roughly subdivide the embryo along the anterior/posterior axis and will regulate the expression pattern of the pair-rule genes. The pair-rule genes divide the embryo into pairs of segments. The pairrule genes encode transcription factors that regulate the expression of the segment polarity genes which will set the anterior/posterior axis of each segment. The gap genes, pair-rule genes, and segment polarity genes are together called the segmentation genes, because they are involved in segment patterning. B) Conservation of homeotic gene clustering, gene order (within the clusters) among organisms from flies to mammals. In addition, the position of homeotic genes on the chromosome is also related to where they are expressed along the anterior/posterior axis (from http://courses.biology.utah.edu).

Homeotic genes have been identified by mutations that change the body plan of the embryo or adult. Mutations in segmentation genes alter the number or pattern of body segments, a part of each segment, or whole groups of segments. Mutations in homeotic genes lead to the development of one body part in the pattern of the other (Fig. 3).

Ed Lewis described the homeotic selector genes of the Antennepedia Complex (lab, $\mathrm{Pb}$, Dfd, Scr and Ant) and the Bithorax Complex (Ubx, abdA and AbdB) that were the master regulators of segmental identity in the fly. Homeotic genes as well as their regulatory regions have a 3' to 5' linear arrangement on the chromosomes that corresponds 
to their expression pattern along the anterior to posterior axis of the fly. Even more significant, this linear arrangement is conserved from flies to humans.

A

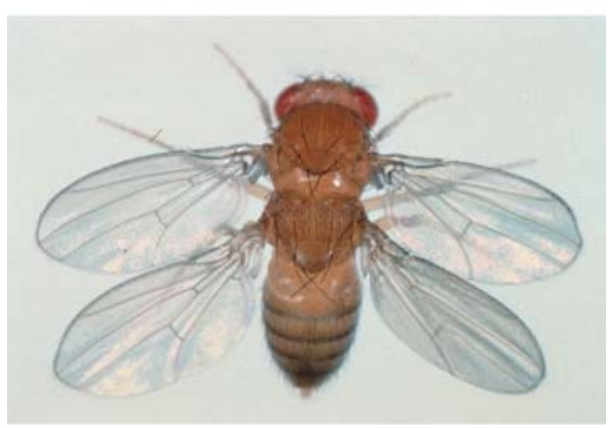

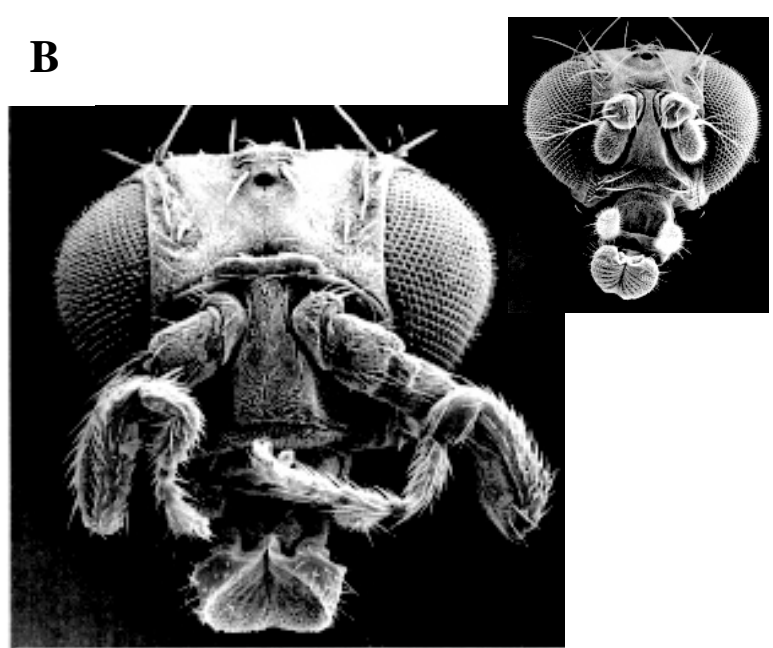

Figure 3. Examples of homeotic mulations. A) Ubx mutation: halteres of the third thoracic segment are transformed into wings that are normally only associated with the second thoracic segment. B) Ectopic expression of Antp leads to transdetermination of discs that normally produce antennae into discs that produce leg structures (photographs of Ant mutants by F. R. Turner, Indiana University)

Upon cloning the Antp gene, Gehring and coworkers have identified the homeobox, a characteristic DNA-segment of 180 basepairs that is shared by all the members of the homeotic gene family. Although the first genes found to encode homeodomain proteins were Drosophila developmental control genes (in particular homeotic genes, from which the name "homeo" box was derived) many homeobox genes are not homeotic genes; the homeobox is a sequence motif, while "homeotic" is a functional description for genes that cause homeotic transformations (Fig. 3). The homeobox encodes a DNA-binding domain, called the homeodomain that is a helix-turn-helix motif (to see the model structure go to the webpage: http://www.homeobox.cjb.net).

\section{I.3 The Polycomb group proteins}

The presence of transcriptional activators and repressors encoded by gap and pairrule genes is only temporary (Gaul and Jäckle, 1989; Tautz, 1988) whereas maintenance of the expression pattern is obligatory. As mentioned above, disturbance of the pattern leads 
to a switch of the determined state and homeotic transformations of the organism (Bienz and Muller, 1995; Busturia and Morata, 1988; Garcia-Bellido et al., 1976; Lewis, 1963). Therefore there must be another mechanism that takes over the responsibility to maintain the correct expression pattern of the homeotic genes. Two groups of proteins were identified to accomplish this task: the Polycomb group (PcG) and Trithorax group (trxG) of proteins. PcG and trxG are chromatin-binding proteins that regulate the expression pattern of many developmental genes (Ringrose, 2003). They are best known to be responsible for conserving the transcriptional state of the homeotic genes and thus, cell identity. PcG proteins are responsible for the persistence of silencing whereas the trxG are required for transcription in the active domains (Francis and Kingston, 2001; Levine et al., 2004; Orlando, 2003). In the history of the PcG proteins there are some major discoveries that led to the present view of their functions: discovery of the $P c$ gene and its importance in the regulation of homeotic genes by Lewis EB in 1978 (Lewis, 1978); characterization of the trithorax gene as a regulator of homeotic gene expression by Ingham PW in 1985 (Ingham, 1985); identification of more PcG and trxG genes and their antagonistic effects in 1988 e.g. (Kennison and Tamkun, 1988);identification of polytene binding pattern of Pc in 1989 (Zink and Paro, 1989); characterization of PREs in Drosophila in 1991-3 ((Fauvarque and Dura, 1993; Muller and Bienz, 1991; Simon et al., 1993); purification of the PRC1 complex in 1999 (Shao et al., 1999); characterization of histone modifications in the mechanism of PcG/trxG proteins 2002-3 (Cao et al., 2002; Czermin et al., 2002; Kuzmichev et al., 2002; Min et al., 2003; Müller et al., 2002).

PcG proteins are targeted to particular regions in the genome called Polycomb response elements (PREs) (Chan et al., 1994; Orlando et al., 1998; Strutt et al., 1997). PcG proteins act in multicomponent protein complexes to repress transcription of their target genes. The continued presence of PcG proteins on the PREs throughout development is required for silencing since deletion of the PRE (Busturia et al., 1997) or individual PcG genes (Beuchle et al., 2001) anytime during organism development results in gene derepression. Interestingly, although PcG complexes maintain the repression pattern for up to 14 cell generations, Buchenau et al. showed that most of the PcG protein complement dissociates at mitosis (Buchenau et al., 1998).

There is experimental data for the association of the PcG proteins with specific chromatin sequences as was first observed by immunofluorescence on polytene chromosomes (Chiang et al., 1995; Franke et al., 1992; Rastelli et al., 1993). In vivo cross- 
linking and chromatin immunoprecipitation (ChIP analysis) of PcG proteins have preferentially detected high levels of proteins of the PCC (Polycomb core complex), and recently, also of Pleiohomeotic (Pho) and Enhancer of zeste $(E(Z))$, on PREs and promoters of known homeobox genes (Breiling et al., 2004; Ringrose et al., 2003; Strutt and Paro, 1997; Wang et al., 2004). Several models have been proposed for the mechanism of PcG-mediated repression such as (a) heterochromatinization or formation of a closed chromatin conformation that does not allow access to promoters; (b) inhibition of the assembly of the preinitiation transciption complex; (c) interference with transcription initiation and/or elongation (Min et al., 2003; Paro and Hogness, 1991; Simon and Tamkun, 2002). Experimental evidence can be found to support each of the models.
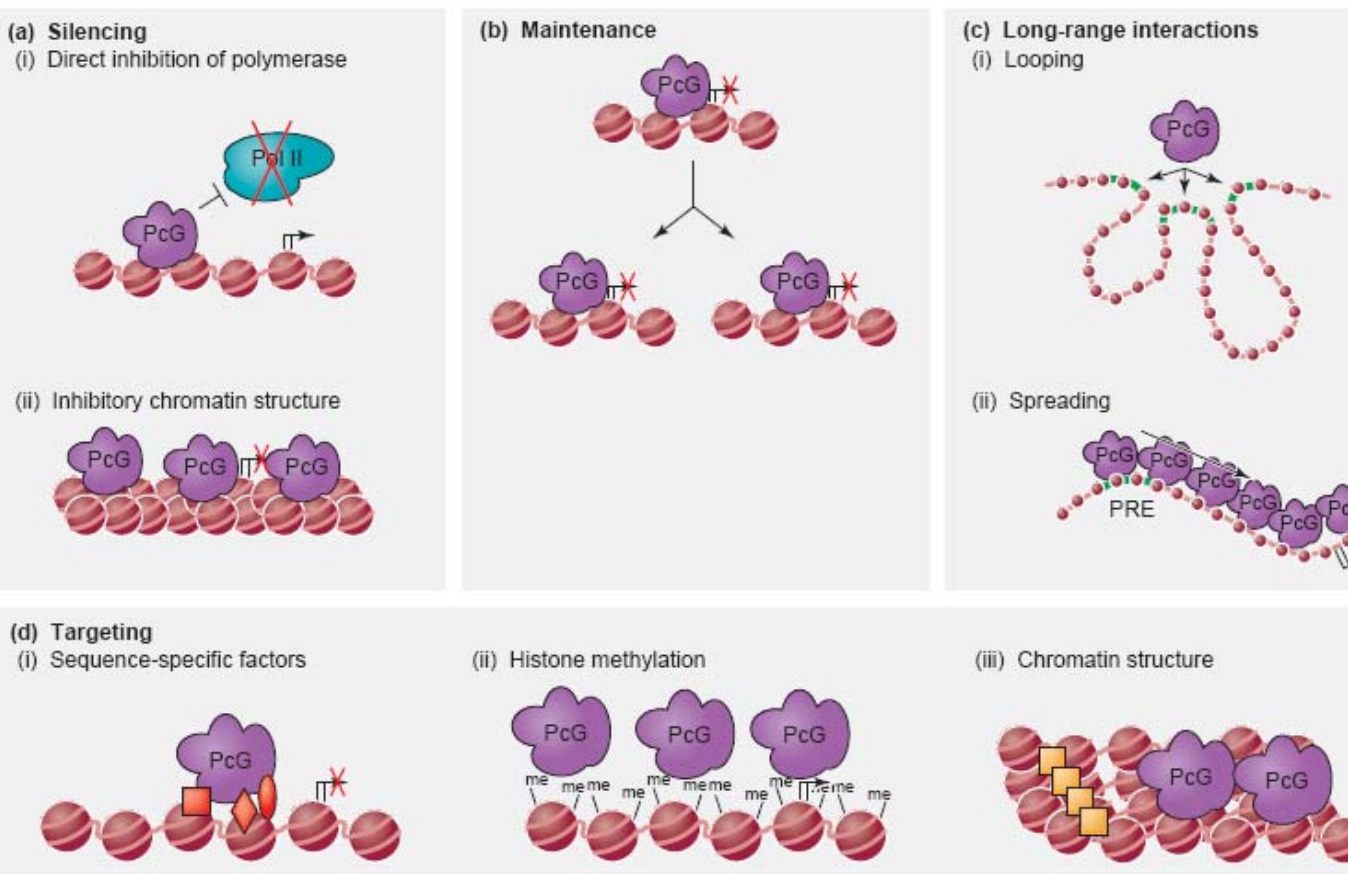

(ii) Spreading

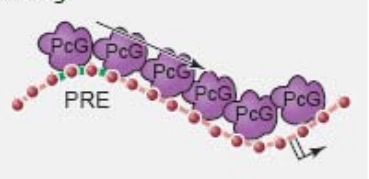

(ii) Histone methylation

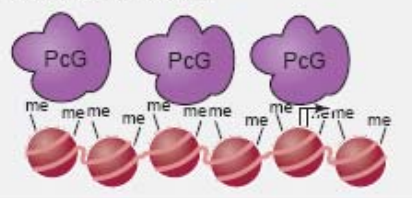

(iii) Chromatin structure

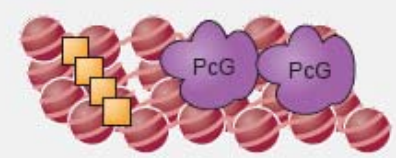

Figure 4. Mechanistic requirements for Polycomb group (PcG) silencing. (a) Transcriptional repression. PcG might inhibit transcription (i) by direct interactions with general transcription factors (green), or (ii) by creating a silenced chromatin structure. (b) Maintenance. The transcriptional state of a PcG repressed gene must be passed on through several rounds of cell division. Genes are likely to be marked for silencing so that PcG repression is maintained through development. (c) Long-range interactions. Interactions between PcG response elements (PREs, green) and silenced promoters (arrows) might be mediated (i) by looping between PREs and promoters, or (ii) by spreading along the chromatin. (d) Targeting. PcG complexes might be targeted (i) by sequence-specific DNA-binding factors (red), (ii) by posttranslational modification of histones, or (iii) by generating a chromatin structure conducive to binding, perhaps mediated by other DNA- 
binding factors that do not interact directly with PcG proteins (orange). Abbreviations: me, methyl groups; Pol II, RNA polymerase II (adapted from Levine SS, 2004).

For example, PcG complexes reduced accessibility over large stretches of DNA in the bithorax homeobox gene cluster (BX-C) for large RNA polymerases (Fitzgerald and Bender, 2001) thereby inhibiting transcription of reporter genes, although restriction enzymes retained DNA access. On the other hand, the presence of PcG proteins at the Ubx promoter in wing imaginal discs (Wang et al., 2004) lends support to a direct inhibition of transcription, though perhaps only to the elongation rather than at the initiation step as has been suggested for the heat shock protein 26 (hsp26) promoter (Dellino et al., 2004).

Table 1 Polycomb group proteins and their function (modified from Levine SS 2004)

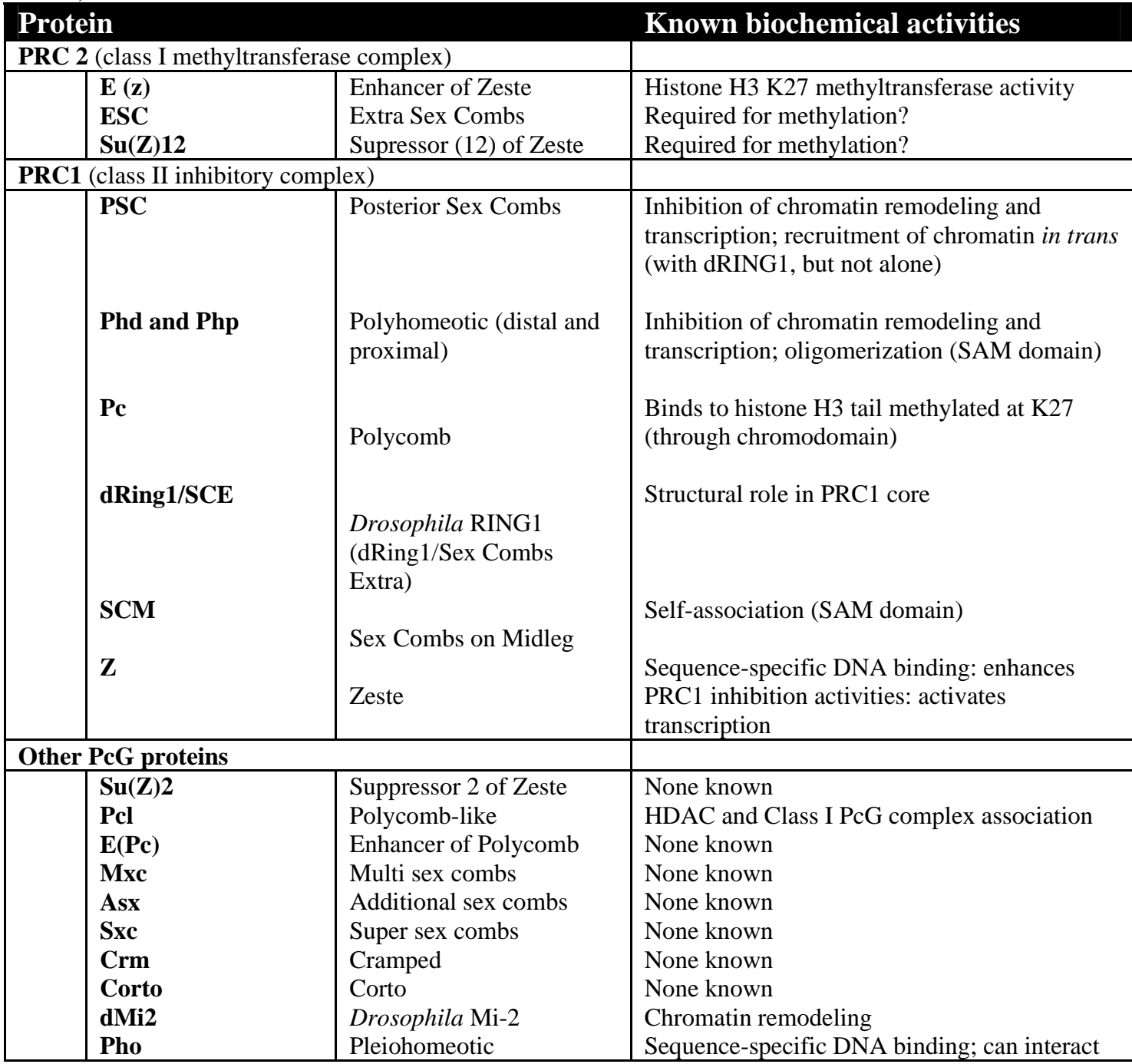




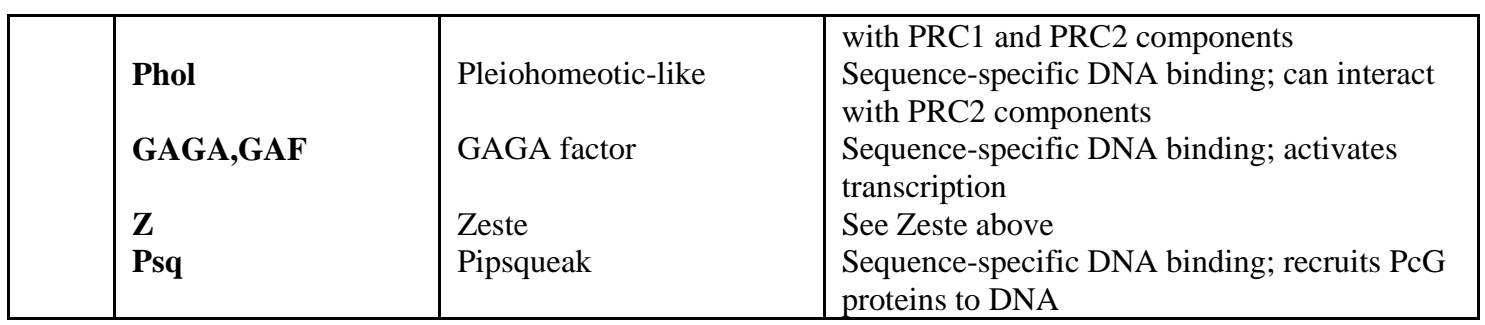

Two different multiprotein polycomb repression complexes (PRCs) have been isolated and characterized biochemically. PRC2, (Ng et al., 2000) is composed of the PcG proteins, Extra sex combs (Esc), Suppressor (12) of zeste (Su(Z)12), and histone-binding Nurf-55 and Enhancer of Zeste (E(Z)), the latter of which methylates histone H3 at lysine 27 both in vivo and in vitro (Cao et al., 2002; Czermin et al., 2002; Kuzmichev et al., 2002; Müller et al., 2002; Yamamoto et al., 2004) thus marking nucleosomes for assembly of repression complexes.

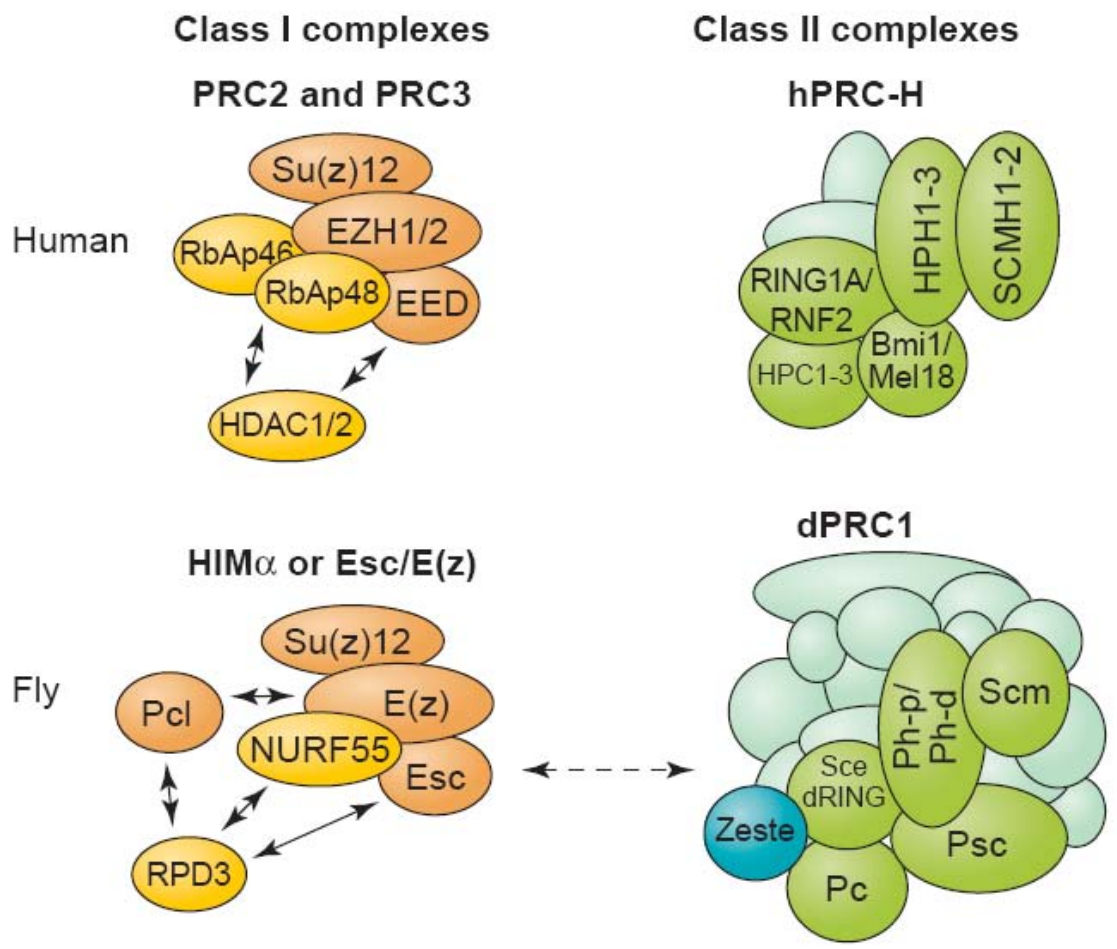

Figure 5. Interactions among Polycomb group (PcG) proteins. Conserved class I and class II PcG proteins are present in complexes in vivo. Orange and green indicate, respectively, class I and class II proteins with known homeotic maintenance phenotypes. Yellow and pale green indicate non-PcG proteins present in each complex. Sequencespecific DNA-binding proteins are indicated in cyan. Double-headed arrows represent physical interactions that are present in only a subset of purified complexes. Broken arrows 
represent known developmentally regulated interactions. Abbreviations: E(Z), Enhancer of Zeste; PRC, Polycomb repressor complex (modified from Levine SS 2004).

PRC1, contains equimolar quantities of Polycomb (Pc), Polyhomeotic (Ph), Posterior sex combs (Psc), and Sex combs extra (Sce/dRing1), all of which have been shown to be essential for PcG silencing. Other PcG and non-PcG proteins such as Sex combs on midleg (Scm), Heat shock protein cognate 4 (Hsc4) and Zeste (Z) and some transcription factors have been isolated with PRC1 in non-stoichiometric amounts which implies the presence of more than one type of polycomb repression complex (Levine et al., 2002; Mulholland et al., 2003; Saurin et al., 2001).

Whether the in vitro isolated or assembled complexes represent truly competent repression machineries is a matter of debate as will be discussed later. In vivo data imply that functional complexes are assembled sequentially on the chromatin itself with a particular hierarchy (Buchenau et al., 1998; Wang et al., 2004) and single PcG gene deficiencies, as noted above, result in homeotic transformations confirming that individual PcG proteins cannot alone cause repression. For a complete understanding of the repression mechanism we need to know the stability and lifetime of functional repression complexes in the living organism. Recently it was reported that Polycomb can be competed away from genomic sites by methylated histone tail peptides in permeabilized salivary gland nuclei (Ringrose et al., 2004). In this study, I have addressed this problem by performing photobleaching experiments (fluorescence recovery after photobleaching, FRAP) on GFP fusion proteins of Polycomb (Pc) and Polyhomeotic (Ph), two essential members of the PCC in whole living Drosophila embryos and larval tissues to determine their diffusion, binding equilibria and residence times. I have measured these values in living organisms at different stages of development to determine whether there are changes in the stability of the complexes. By taking advantage of the polytene nature of the salivary gland chromosomes I was able to address the question of the uniformity of the complexes by investigating the behaviour of the protein on individual bands. I was able to extract data that allow us to fit the actual forward and reverse rate constants for complex formation. Most of the complexes exchange within a period of a minute and all of the complexes within 10 minutes. I discuss how compatible these data are with present models for repression as well as what can be inferred about homogeneity of the repression complex. 


\section{I.4 Photobleaching microscopy and mobility of molecules inside cells}

The activity of genes in eukaryotic organisms is modulated by non-histone chromatin binding proteins. Both global and local chromatin states can be transmitted from one cell generation to another (Grewal and Moazed, 2003; Phair et al., 2004; Vermaak et al., 2003). However, data on the lifetime of chromatin-bound protein complexes and an understanding of the actual mechanisms by which they exert their activation or repression are lacking. Photobleaching techniques such as FRAP (fluorescent recovery after photobleaching) introduced in the 70's were initially restricted to lateral diffusion measurements on the cell membrane (Axelrod et al., 1976; Edidin et al., 1976; Jacobson et al., 1976; Peters et al., 1974). However, advanced in vivo microscopy methods combined with expression vectors for visible fluorescent fusion proteins (VFP) now provide the means to study the functionality and the physical properties of molecules throughout the whole cell or animal. (reviewed in (Lippincott-Schwartz et al., 2003; Lippincott-Schwartz and Patterson, 2003)). In particular, FRAP studies of the dynamics of VFP-labelled nuclear proteins in living cells has changed the view of the nucleus (Catez et al., 2004; Misteli, 2001).

Several photobleaching methods can be applied depending on the question asked and the complexity of the biological conditions: FRAP, iFRAP (inverse FRAP), FLIP (fluorescence loss in photobleaching), half-FRAP (see Materials and Methods section for detailed explanations). Most of the FRAP experiments on VFP-fusion proteins in the cytoplasm or nucleus have been aimed at investigating binding reactions or the stability of complexes. In many of the earlier experiments a qualitative analysis of the recovery curves has been presented (Dou et al., 2002). More recently a number of groups have analyzed their data using kinetic parameters for chemical equilibria and binding reactions (Carrero et al., 2003; Dundr et al., 2004; Dundr et al., 2002; Phair and Misteli, 2000; Phair et al., 2004; Stavreva et al., 2004).

In a global analysis of the binding properties of a number of nuclear proteins, Catez et al. adopted a classical compartmental model (Catez et al., 2004). They used half-FRAP, a photobleaching method whereby half of the nucleus is bleached and recovery of the signal in the bleached region and loss of signal in the unbleached region are evaluated over time. Although this method proved to be very useful in comparing the binding properties of a number of transcription factors and chromatin proteins under the same experimental 
conditions, the compartment model is not accurate enough and not applicable to all chromatin binding proteins. Furthermore, the influence of diffusion to the recovery time was completely ignored in the analysis. I show here that diffusion plays an important role in the binding process and will influence significantly the measured dissociation rate constant. Catez et al. extracted binding constants by fitting the recovery curves to a double exponential function describing a fast component and a slow component. Of particular importance is the second assumption that the concentration of binding sites is equal throughout the whole nucleus, a condition that is not applicable for many chromatin binding proteins which localize in discrete spots.

In the study of Carrero et al., a similar compartmental model was described. The authors treated diffusion as a rate constant in a compartmental ad-hoc model without showing the validity of the assumption (Carrero et al., 2003). In analyzing the exchange rates of proteins of the nuclear pore complex Rabut and Ellenberg also did not account for diffusion (Rabut et al., 2004).

Spague et al. have presented a full analytical solution for binding reactions studied by FRAP using a circular bleach area (Sprague et al., 2004). This model is a detailed analysis of a single binding interaction in the presence of diffusion but it can also be extended to cases with multiple, independent binding interactions. Three idealized solutions are described: a pure diffusion-dominant, a combined diffusion and binding case "effective diffusion" and a reaction dominant case. For the full model the authors derived an analytical solution for the Laplace transform of the FRAP curve with respect to time. An important assumption in this model is that there is an equal concentration of binding sites over the whole nucleus. However, in many biological systems binding sites or protein complexes are distributed inhomogeneously.

I have collaborated with theoreticians to develop a model for the binding kinetics of the PcG proteins and to describe this mathematically which I present in this thesis. The model was used to simulate the binding reactions "in silico", and helped to distinguish the physical processes underlying photobleaching experiments and their limits (see Results). This information has been used to interpret the FRAP experimental results. The fitting algorithms for binding take into account diffusion, a factor, which would lead, if ignored, to erroneous interpretation of the data. The usefulness of the model can be extended to other biological systems where proteins have localized target sites (e.g. other chromatin binding proteins, cytoskeleton-, membrane binding proteins etc.). The simulation program 
is able to extract data from the imaged cell or nucleus and according to other input parameters (like diffusion constant calculated separately) it can simulate the binding reaction and return the kinetic constants.

\section{I.5 Computer simulations - a means to obtain a better insight into cellular processes}

Biological systems and the physics of the experiment often exhibit a degree of complexity, which do not allow a direct interpretation of the data. It may be useful to make specific hypotheses and use the help of computer simulations to see whether a suggested model of the biological system is consistent with the experimental data. An example in this direction is the use of computer simulations in calculating the evolution of a set of dynamic filaments with motor proteins (Nedelec, 2002). This was done to examine how two asters of dynamic microtubules nucleated by two microtubule organizing centers can reach a steady-state configuration in which microtubules overlap fully or partially. Such overlaps are essential in some spindles to counteract the forces that pull the chromosomes apart. On the other hand simulations often help to predict a particular biological behavior. For example: interaction of a protein with other molecules (ligands or other protein) relies on the process of three dimensional folding of the protein that would expose particular structural motifs recognized by other small molecules. A single mutation changing one amino acid in a protein can be enough to cease this interaction. To understand such effects, molecular biologists study the interaction of a protein with other molecules, which may be small ligands or other proteins. They can identify a region of tens or a hundred amino acids which are responsible for some specific action. Ab initio simulation can then pinpoint more precisely where and how the interaction actually works and what effect the mutation has.

Also in FRAP many biophysical processes are involved. With major simplifications it is possible to obtain analytical models of the system, obviating the need for computer simulations. However, when these simplifications cannot be made or they are at least questionable, computer simulations accounting in more detail for the different processes and constraints can be useful. They can even help in the process of simplification by indicating which parameters can safely be omitted or linked. 


\section{MATERIALS AND METHODS}

\section{II.1 Molecular biology materials and methods}

\section{II.1.1 Materials}

\section{II.1.1.1 Chemicals and enzymes}

All chemicals used in this work are of molecular biology quality and were acquired from Invitrogen ${ }^{\mathrm{TM}}$, Roche Diagnostics, Sigma Aldrich or Merck. Suppliers for restriction endonucleases were Roche, NEB (New England Biolabs, Boston, MA) and Hybaid. DNA polymerases and ligases were from Promega or included in the Expand High Fidelity PCR System from Roche and in the Rapid DNA Ligation Kit from Roche, respectively. Schrimp alkaline phosphatases were purchased from Roche. Additional commercial kits used were described in the following sections.

\section{II.1.1.2 Buffers and solutions}

$\begin{array}{llll}\text { PBS: } & \mathrm{NaCl} & 137 \mathrm{mM} & \mathrm{pH} 7.3 \\ & \mathrm{KCl} & 2.7 \mathrm{mM} & \\ & \mathrm{Na}_{2} \mathrm{HPO}_{4} \mathrm{x}_{2} \mathrm{H}_{2} \mathrm{O} & 7.9 \mathrm{mM} & \\ & \mathrm{KH}_{2} \mathrm{PO} 4 & 1.5 \mathrm{mM} & \\ & & & \\ \text { PBST: } & 1 \times \mathrm{PBS} & +0.2 \% \text { Tween20 } & \\ & & & \\ \text { TAE-buffer: } & \text { Tris pH } 8.0 & 2 \mathrm{M} & \\ \text { (50x) } & \text { Acetic acid gl. } & 1 \mathrm{M} & \\ & \text { EDTA } & 50 \mathrm{mM} & \mathrm{pH} 7.2 \\ & & & \\ \text { Tyrode’s buffer } & \text { NaCl } & 135 \mathrm{mM} & \\ \text { (RAB) } & \text { KCl } & 10 \mathrm{mM} & \\ & \text { MgCl2 } & 0.4 \mathrm{mM} & \\ & \text { CaCl2 } & 1 \mathrm{mM} & \\ & \text { HEPES } & 10 \mathrm{mM} & \\ & \text { Glucose } & 5.6 \mathrm{mM} & \end{array}$

These are standard buffers. Other buffers are described in the Methods section. 


\section{II.1.1.3 Bacterial hosts, media and culture plates}

Strain

$\mathrm{DH} 5 \alpha$

Chemically competent

\section{Genotype}

SupE, Alac, hsdR, recA, endA, gyrA, thi, relA

Additional bacterial hosts were the TOP10 chemically competent E. coli (Invitrogen).

$\begin{array}{lll}\text { LB broth medium } & \text { Bacto tryptone } & 10 \mathrm{~g} \\ \text { 1L } & \text { Bacto yeast extract } & 5 \mathrm{~g} \\ & \mathrm{NaCl} & 10 \mathrm{~g} \\ & \mathrm{H}_{2} \mathrm{O} & \text { up to } 1 \mathrm{~L} \\ & & \\ \text { SOC medium } & \text { Bacto tryptone } & 20 \mathrm{~g} \\ 1 \mathrm{~L} & \text { Bacto yeast extract } & 5 \mathrm{~g} \\ & \mathrm{NaCl} & 0.5 \mathrm{~g} \\ & \mathrm{KCl}, 1 \mathrm{M} & 2.5 \mathrm{~mL} \\ & \mathrm{H}_{2} \mathrm{O} & \text { up to } 1 \mathrm{~L} \\ & \mathrm{MgCl}, 2 \mathrm{M} & 5 \mathrm{~mL} \text { (added after autoclaving) } \\ & 50 \% \text { Glucose in water } & 10 \mathrm{~mL} \\ & & \\ & \text { LB medium } & 1 \mathrm{~L} \\ \text { LB agar (plates) } & \text { Bacto-agar (Gibco-BRL) } & 15 \mathrm{~g} \text { (added before autoclaving) } \\ \text { 1L } & \end{array}$

After autoclaving and cooling, ampicillin $(50 \mu \mathrm{g} / \mathrm{mL})$ or kannamycin $(100 \mu \mathrm{g} / \mathrm{mL})$ were added to the media.

\section{II.1.1.4 Vectors}

For the EGFP sequence I used the pEGFP-N1 vector (BD Biosciences, US) with the multiple cloning site at the $\mathrm{N}$ terminus of the EGFP sequence. PCR fragments were cloned into the TOPO T/A Cloning vector, a component of the standard T/A cloning kit from Invitrogen. For generating transgenic fly lines, promoter and gene coding sequences were cloned into pUAST. This vector is derived from pCaSpeR3, a P element vector that contains the P element ends (P3' and P5') and the white gene which acts as a marker for successful incorporation into the Drosophila genome. In addition pUAST contains five tandemly arrayed optimized GAL4 binding sites followed by the hsp70 TATA box. As a 
transposase source the P-element helper plasmid pUChspD2-3 carrying the transposase gene was used.

\section{II.1.1.5 Machines and software}

- Cecil spectrophotometer series 6000: for DNA and protein quantification

- DNASTAR Lasergene v6: for DNA and protein sequence analysis

- GS 670 and 710 densitometers (Biorad, CA): for quantification of western blots

- Molecular analyst/Quantity one: software for quantification of western blots

\section{II.1.2 Molecular biology and biochemistry methods}

\section{II.1.2.1 Plasmid DNA purification from E. coli and determination of DNA concentration}

Isolation of plasmid DNA was performed according to the manufacturer's handbook using buffers and equipment contained in the kit (QiaQuick Plasmid Mini Kit and QiaFilter Plasmid Midi Kit, Qiagen, Hilden). For preparative purposes $5 \mathrm{~mL}$ (miniprep) and $200 \mathrm{~mL}$ of liquid medium were inoculated with 1:1000 dilution from an overnight preculture and incubated overnight at $37^{\circ} \mathrm{C}$ on a shaker.

DNA concentration was calculated as following:

$$
C(\mu g / \mu L)=\frac{A_{260} x d}{\varepsilon_{D N A}} \cdot \frac{1}{p(\mathrm{~cm})}
$$

where $A_{260}$ is the absorption of DNA at $260 \mathrm{~nm}, d$ is the dilution factor, $\varepsilon_{D N A}=20$ is the extinction coefficient of DNA and $p$ is the path length of the cuvette.

\section{II.1.2.2 Agarose gel electrophoresis and DNA elution from gels}

Analysis and purification of DNA fragments was performed by agarose gel electrophoresis. Typically, $0.7 \%$ agarose was prepared in $1 \mathrm{x}$ TAE buffer with $0.5 \mu \mathrm{g} / \mu \mathrm{L}$ ethydium bromide (BioLogical ${ }^{\mathrm{TM}}$ ) for DNA visualization under UV light with excitation at 
$312 \mathrm{~nm}$. DNA samples were mixed 1:50 with 50x Bromphenol Blue (BioRad, in 50\% glycerol) and separation was done at $100 \mathrm{~V}$ at room temperature (RT). $1 \mathrm{~kb}$ DNA ladder (New England Biolabs, Boston, MA) was used as a standard marker (10 $\mu \mathrm{L}$ of $50 \mathrm{ng} / \mu \mathrm{L}$ solution). Extraction of the band of interest was done under UV light using a clean scalpel. Elution of DNA from the gel was done with the QIAquick Gel Extraction Kit (Qiagen) following the manufacturer's protocol in $50 \mu \mathrm{l}$ water.

\section{II.1.2.3 Polymerase chain reaction (PCR)}

For in vitro amplification of DNA, the polymerase chain reaction was used. The reaction is a cyclic process where short sequences of nucleotides (oligonucleotides or primers) anneal specifically to both ends of a particular denatured DNA sequence followed by addition of single nucleotides by the DNA Polymerase III enzyme until a new sequence is obtained. Typical PCR reaction mixtures and cycling programs used:

\begin{tabular}{|c|c|c|c|c|c|c|}
\hline \multicolumn{3}{|c|}{ Reaction mixture (for $100 \mu \mathrm{L}$ ) } & \multicolumn{4}{|c|}{ Cycling program } \\
\hline Template DNA & $0.2 \mu \mathrm{L}$ & $\begin{array}{l}50-500 \\
\mathrm{ng} / \mu \mathrm{L}\end{array}$ & \multicolumn{2}{|c|}{ Denaturation I } & $95^{\circ} \mathrm{C}$ & $2 \min$ \\
\hline 10x buffer & $10 \mu \mathrm{L}$ & & \multirow{3}{*}{ 官 } & \multirow{3}{*}{$\begin{array}{l}\text { Denaturation II } \\
\text { Primer extension } \\
\text { Annealing }\end{array}$} & \multirow{3}{*}{$\begin{array}{l}95^{\circ} \mathrm{C} \\
50-65^{\circ} \mathrm{C} \\
72^{\circ} \mathrm{C}\end{array}$} & \multirow{3}{*}{$\begin{array}{l}45 \mathrm{sec} \\
30 \mathrm{sec} \\
1 \mathrm{~min} / \mathrm{kb}\end{array}$} \\
\hline dNTPs & $2 \mu \mathrm{L}$ & $40 \mathrm{mM} \operatorname{mix}$ & & & & \\
\hline Primer 1 & $5 \mu \mathrm{L}$ & $10 \mu \mathrm{M}$ & & & & \\
\hline Primer 2 & $5 \mu \mathrm{L}$ & $10 \mu \mathrm{M}$ & \multirow{2}{*}{\multicolumn{3}{|c|}{$\begin{array}{l}\text { For PRC product insertion into pCR II } \\
\text { add } 1 \mu \mathrm{L} \text { of Taq Polymerase }\end{array}$}} & \multirow{2}{*}{$\begin{array}{l}72^{\circ} \mathrm{C} \\
10 \mathrm{~min}\end{array}$} \\
\hline Water & $77 \mu \mathrm{L}$ & & & & & \\
\hline Enzyme (Pfu) & $0.8 \mu \mathrm{L}$ & & \multicolumn{3}{|c|}{ Hold at $4^{\circ} \mathrm{C}$} & \\
\hline
\end{tabular}

When amplifying the ESC promoter and gene from the Drosophila genome, denaturation prior the cycles (28) was done for 4 minutes, annealing temperature was at $50^{\circ} \mathrm{C}$ and the polymerase used was Long Expand Polymerase (Roche Diagnostics). Amplification with the Pfu in this case did not give a result. 


\section{II.1.2.4 Primer design and cloning strategy}

DNA sequences for the Pc promoter, Ph gene, GFP, ESC promoter and ESC gene were analyzed with the Lasergene DNA analysis software.

The $\mathrm{Ph}$ (proximal) gene was fused to green fluorescent protein (PhGFP) using the strategy described in Netter et al. (2001). It was cloned with both a UAS and a Pc promotor to provide controlled expression at different stages of fly development. The predicted $P_{C}$ promotor (Neural Network Promoter prediction program of the Berkeley Drosophila Genome Project) was isolated from a Pst I fragment of the $P_{c}$ genomic clone (kind gift of Jürg Müller) (Paro and Hogness, 1991) by amplification of a 559 basepair (bp) fragment using the primers listed below P1 and P2 ( and inserted as an EcoRI fragment upstream of the $P h$ protein coding sequence (kind gift from Jürg Müller). The resulted fusion was inserted as a BglII-NotI fragment into the pUAST vector (Brand and Perrimon, 1993).

The esc promoter from the $0.26 \mathrm{~kb}$ upstream sequence to the genomic esc gene fragment (Gutjahr et al., 1995) was amplified from the genome using the primers P1 and P2 (listed below) as a KpnI-HindIII fragment. The 5' primer to amplify the GFP sequence contained a HindIII site followed by an ATG transcription start codon and the 3' primer was designed to exclude the stop-codon followed by an EcoRV restriction site. The full length esc gene was amplified from the genome with a 5' primer containing an EcoRV site followed by the second codon of the gene and a 3' primer terminating with an XbaI restriction site. In this way the GFP is in frame with the esc sequence and the resulted linker sequence is: GATATCGCA (or as protein sequence: D-I-A). After ligating all sequences together, the resulted KpnI-XbaI DNA fragment (containing the esc promoter, GFP and the esc gene sequence) was inserted in the pUAST P-element vector.

Transgenic lines containing the construct $P[U A S, P c: P h G F P]$ were generated with standard transformation protocols using the $w^{1118}$ host line (Spradling and Rubin, 1982) and the site of chromosome integration was determined genetically.

The sense and antisense primers (see below) were designed to flank the sequences above and contained extra restriction sites for subsequent subcloning of the PCR product into appropriate plasmids. 


\begin{tabular}{|l|l|l|}
\hline \multirow{2}{*}{ Name } & \multicolumn{2}{|l|}{ Primer sequence (5'-3') } \\
\hline \multirow{2}{*}{ Pc promoter } & P1 & TTT AGA TCT CAA TTT GTG ATA CAA TAA GTG \\
\cline { 2 - 3 } & P2 & CCC GAG CTC ATC TTA GCA AGT AGC CGT GTC \\
\hline \multirow{2}{*}{ ESC promoter } & P1 & TTT GGT ACC AGT CTT GTC GAC CAG TGT AAT \\
\cline { 2 - 3 } & P2 & TTT AAG CTT TTC GAA CGC GCG GCA CGT TTG \\
\cline { 2 - 3 } & P1 & TTT GAT ATC GCA AGC AGT GAT AAA GTG AAA \\
\hline GFP (for ESC) & P1 & GGG AAG CTT ATG GTG AGC AAG GGC \\
\cline { 2 - 3 } & P2 & GGG ATA TCC TTG TAC AGC TCG TCC \\
\hline
\end{tabular}

\section{II.1.2.5 Restriction enzyme digestion of DNA and ligation reactions}

Restriction endonucleases are bacterial enzymes that bind and cleave DNA at specific target sequences. Type II restriction enzymes bind DNA at a specific recognition site, consisting of a short palindromic sequence, and cleave within this site. Restriction enzyme digestion of DNA was done for analytical (to identify the right sequence and the correct insertion of a DNA fragment) and preparative purposes (for large scale production of a DNA fragment).

Preparative digest (50 $\mu \mathrm{L}$ final volume): $10 \mu \mathrm{g}$ DNA (from miniprep), $5 \mu \mathrm{L} 10 \mathrm{x}$ buffer (for enzymes A and B), $2 \mu \mathrm{L}$ enzyme A, $2 \mu \mathrm{L}$ enzyme B, $5 \mu \mathrm{L} 10 \times$ BSA (when required), $\mathrm{H} 2 \mathrm{O}$ to a final volume of $50 \mu \mathrm{L}$. Incubation was done for at least $2 \mathrm{~h}$ at $37^{\circ} \mathrm{C}$ followed by agarose gel purification.

Analytical digest (10 $\mu \mathrm{L}$ final volume): $0.5 \mu \mathrm{L}$ DNA (from miniprep $\sim 0.5 \mu \mathrm{g} / \mu \mathrm{L}$ ), $1 \mu \mathrm{l} 10 \mathrm{x}$ buffer (for enzymes A and B), $0.2 \mu \mathrm{L}$ enzyme A, $0.2 \mu \mathrm{L}$ enzyme B, $1 \mu \mathrm{L} 10 \mathrm{x}$ BSA (when required), H2O to a final volume of $10 \mu \mathrm{L}$. Incubation was done for 30-60 minutes at $37^{\circ} \mathrm{C}$ followed by agarose gel migration.

Ligation of DNA fragments involves creating a phosphodiester bond between the 3' hydroxyl of one nucleotide and the 5' phosphate of another. The enzyme used to ligate DNA fragments is T4 DNA ligase, which originates from the T4 bacteriophage. This enzyme will ligate DNA fragments having overhanging, cohesive ends that are annealed 
together. DNA ligation was performed with the Rapid DNA ligation kit (Roche Diagnostics). Composition of ligation solution: $200 \mathrm{ng}$ of total DNA (1:3 molar ratio insert:vector), $1 \mu \mathrm{L} 5$ x Buffer $2, \mathrm{H}_{2} \mathrm{O}$ up to $5 \mu \mathrm{L}$ total volume. Composition was mixed by vortexing and $5 \mu \mathrm{L} 2$ x ligation Buffer 1 was added to it. After mixing the solution, $0.5 \mu \mathrm{L}$ ligase was added to perform the ligation reaction at RT for 5 minutes.

\section{II.1.2.6 Transformation of competent bacteria}

Chemically competent $\mathrm{DH} 5 \alpha$ or TOP10F' bacterial cells have been used for plasmid DNA amplification. Transformation was performed with the heat shock method. A $100 \mu \mathrm{l}$ aliquot of competent bacteria was thawed on ice and mixed with the ligation solution (see above). After 30 minutes incubation cells were heat shocked at $42^{\circ} \mathrm{C}$ for exactly 90 seconds and subsequently cooled for 2 minutes on ice. $900 \mu \mathrm{l}$ of complete medium (LB for DH5 $\alpha$ or SOC for TOP10F') were added followed by 1 h incubation at $37^{\circ} \mathrm{C}$ on a shaker (to recovery bacteria). To distinguish bacteria that were successfully transformed the cells were transferred to ampicillin of kannamycin containing solid agar. Only transformants could grow on this medium, because resistance against the antibiotic is located only on the transformed vector.

\section{II.1.2.7 Total protein extraction from Drosophila tissues}

Crude extracts from Drosophila embryos and larval tissues were prepared for analytical purposes. Embryos from overnight collections or from various developmental stages (0-3, 3-6, 6-12, 12-21) were dechorionated (3 minutes in 50\% Klorix), washed with embryo wash solution $(0.4 \% \mathrm{NaCl}, 0.03 \%$ Triton $\mathrm{X}-100)$ and placed into $2 \mathrm{~mL}$ round bottom eppendorf tubes (0.02 $\mu$ g embryos/cup). 50 $\mu \mathrm{L}$ Drosophila tissue lysis buffer (DLB) was added to the embryos. Explanted larval tissues (imaginal discs and salivary glands) were shortly washed in PBT and placed into $2 \mathrm{~mL}$ round bottom eppendorf tubes with 40 $\mu \mathrm{L}$ Drosophila tissue lysis buffer (DLB) (15-30 tissues/cup). Embryos and tissues in DLB were frozen $\left(-20^{\circ} \mathrm{C}\right)$ or immediately subjected to protein extraction (see below).

\section{Protocol for total protein extraction (continuation):}

- Squash embryos or tissues with a clean pestle for round bottom eppendorf tubes by rotating and moving the pestle up and down for 30 seconds 
- Freeze the tube for 10 seconds in liquid nitrogen

- Repeat the freezing-thawing-squashing procedure 3-4 times

- $\quad$ Rinse the pestle with 20-50 $\mu \mathrm{L}$ DLB

- Mix the solution by vortexing for 1 second

- Remove 7-10 $\mu \mathrm{L}$ from the extract for protein concentration measurement

- $\quad$ Measure extract volume $(\sim 70-100 \mu \mathrm{L})$

- $\quad$ Add 4x NuPage LDS Sample Buffer (Invitrogen)

- Add DTT to a final concentration of $50 \mathrm{mM}$ (from 0.5 or $1 \mathrm{M}$ stock solution)

- Boil the extract for 10 minutes at $70^{\circ} \mathrm{C}$ or 5 minutes at $95^{\circ} \mathrm{C}$

- Centrifuge the extract for 10 minutes

- Separate the water soluble fraction from the lipid layer (especially when extracting proteins from embryos)

- $\quad$ Aliquote extract (30-50 $\mu \mathrm{L}$ each)

\section{Drosophila tissue lysis (DLB) buffer composition:}

$\begin{array}{llll}\text { Lysis buffer composition } & \text { Concentration } & \text { Stock solution } & \text { Amount for } \mathbf{1 4} \boldsymbol{\mu L} \\ \text { Hepes-KOH pH } 7.5 & 20 \mathrm{mM} & 1 \mathrm{M} & 0.28 \mathrm{~mL} \\ \mathrm{KCl} & 100 \mathrm{mM} & 1 \mathrm{M} & 1.4 \mathrm{~mL} \\ \text { EDTA } & 2 \mathrm{mM} & 0.5 \mathrm{M} & 56 \mu \mathrm{L} \\ \text { Triton X-100 } & 0.5 \% & 10 \% & 700 \mu \mathrm{L} \\ \text { Aprotinin } & 0.3 \mathrm{U} / \mathrm{mL} & & 4.2 \mathrm{U} \\ \text { Leupeptin } & 10 \mu \mathrm{g} / \mathrm{mL} & & 140 \mu \mathrm{g} \\ \text { Soy bean trypsin inhibitor } & 100 \mu \mathrm{g} / \mathrm{mL} & & 1.4 \mathrm{mg} \\ \text { Complete inhibitor tabs } & 1 \mathrm{Tab} / 7 \mathrm{~mL} & & 2 \mathrm{tabs} \\ \text { DTT } & 5 \mathrm{mM} & 0.5 \mathrm{M} & 140 \mu \mathrm{L} \\ \mathrm{Mg}\left(\mathrm{CH}_{3} \mathrm{COO}\right)_{2} & 1 \mathrm{mM} & 1 \mathrm{M} & 14 \mu \mathrm{L} \\ \mathrm{H}_{2} \mathrm{O} & & & 10.68 \mathrm{~mL}\end{array}$




\section{Measuring total protein concentration:}

- Prepare a standard curve using a BSA solution $(1 \mu \mathrm{g} / \mu \mathrm{L})$

$\begin{array}{lllllllllll}\text { Water }(\mu \mathrm{L}) & 20 & 19 & 18 & 17 & 16 & 15 & 14 & 12 & 10 & 8 \\ \text { BSA }(\mu \mathrm{L})(1 \mu \mathrm{g} / \mu \mathrm{L}) & 0 & 1 & 2 & 3 & 4 & 5 & 6 & 8 & 10 & 12\end{array}$

- Make two dilutions of the extract $(2 \mu \mathrm{L}$ and $4 \mu \mathrm{L}$ extract in 18 and $16 \mu \mathrm{L}$ water, respectively). When possible, use duplicates for each dilutions

- Mix BSA and extract dilutions with $1 \mathrm{~mL}$ 1:5 diluted 5x Bio-Rad protein assay solution (BioRad Laboratories, München). The Bio-Rad Protein assay is a procedure based on the method of Bradford for determining concentration of solubilized protein

- Incubate at room temperature for at least 5 minutes. Absorbance will increase over time; samples should incubate at room temperature for no more than 1 hour.

- Measure absorbance at $595 \mathrm{~nm}$.

- Calculate total protein concentration of the extract using the standard curve.

\section{II.1.2.8 Polyacrylamide gel electrophoresis (SDS-PAGE)}

Proteins migrate in an electric field at rates that depend on their net electric charge. Protein separation was carried out using the commercially provided XCell SureLock ${ }^{\mathrm{TM}}$ SDS-PAGE system (Invitrogen). Therefore, migrations were carried out in the presence of denaturing agents; in this case, the tertiary and quaternary structures of proteins are destroyed and the resulting protein subunits are separated. The most widely used denaturant at the present time is the anionic detergent, sodium dodecyl sulfate (SDS), which disassociates virtually all protein-protein, protein-nucleic acid and protein-lipid mixtures. (When sodium ion concentration must be controlled, Lithium dodecyl sulfate LDS - is substituted). Separation of proteins with lower molecular weight (20-100 kDa, Pc and PcGFP) was carried out in pre-cast gels with a gradient concentration of acrylamide of 4-12\% (NuPAGE TM 4-12\% Bis-Tris Gel) whereas for proteins with high molecular weight (>150 kDa, PhGFP), Novex ${ }^{\circledR}$ NuPAGE тм 3-8\% Tris Acetate Gel has been used. For running buffers, NuPAGE® MES SDS Running Buffer and Novex TM Tris-Acetate SDS Running Buffer were used, respectively. Protein samples mixed with sample buffers were 
loaded after boiling at $70^{\circ} \mathrm{C}$ for 10 minutes. Generally, $4-10 \mu \mathrm{g}$ of total proteins were loaded per well. SeeBlue ${ }^{\circledR}$ Plus2 Pre-Stained was used for molecular weight marker. Electrophoresis was carried out at a constant voltage of $180 \mathrm{~V}-1$ hour for $4-12 \%$ Bis-Tris gels and constant $150 \mathrm{~V}-3$ hours for 3-8\% Tris Acetate gels. After electrophoresis the gel was used for western blotting experiments.

\section{II.1.2.9 Immunoblotting and proteins quantification on western blots}

The electrophoretic transfer of proteins from sodium dodecyl sulphate polyacrylamide gels (SDS-PAGE) to sheets of nitrocellulose is referred to as Western Blotting or immunoblotting. It allows determining, with a specific primary antibody, the relative amounts of the protein present in different samples. A standard semidry western blotting was carried out using nitrocellulose membranes (Optitran BA-S 83, Schleicher \& Schuell, Germany). Proteins were transferred for 35 minutes (low MW ( $<100 \mathrm{kDa}$ )) or 2 hours (higher MW (100-200 kDa)) at $1.5 \mathrm{~mA} / \mathrm{cm}^{2}$ (110 mA/pre-cast minigel). For higher molecular weight proteins, a bigger surface of Whatman paper was soaked in electroblotting buffer and evaporation was protected by covering the surface of the Whatman paper around the nitrocellulose membrane with Parafilm ${ }^{\circledR}$ (Chicago, IL). Immunoblots were blocked in $10 \%(\mathrm{v} / \mathrm{v})$ heat inactivated fetal calf serum (Sigma) in PBT (30 minutes) to block unspecific protein binding and probed with primary polyclonal antiPc (kind gift of R. Paro), anti-Ph or anti-GFP in dilutions specified in Table 2. Primary antibody incubation was carried out for 2 hours at $37^{\circ} \mathrm{C}$ or overnight at $4^{\circ} \mathrm{C}$. Before applying the secondary antibodies in blocking buffer, blots were washed $3 \mathrm{x}$ in PBT. After 1 hour incubation with HRP-conjugated secondary antibodies chemiluminescence was measured with the ECL detection kit (Amersham Pharmacia Biotech). PABP (Roy G., 2004), S6 (Santa Cruz Biotehnology, Inc) and eIF4A (kind gift of Hernandez G., 2004) antibodies were used as loading controls. The intensity of the signals on the x-ray films was quantified on a scanning densitometer (G-710, BioRad). O.D. values were extracted and normalized to the proteins indicated in Table 3. 
Table 2. Primary antibodies

\begin{tabular}{|l|l|l|}
\hline Antibody & Dilution & Reference \\
\hline Polycomb & $1: 10000$ & $\begin{array}{l}\text { Serum (kind gift from Renato } \\
\text { Paro) }\end{array}$ \\
\hline Polyhomeotic & $1: 3000$ & (Buchenau et al., 1998) \\
\hline aGFP2 & $1: 500$ & Serum (produced in this lab) \\
\hline PABP & $1: 5000$ & Roy G., 2004 \\
\hline S6 & $1: 500$ & Santa Cruz Biotehnology, Inc \\
\hline eIF4A & $1: 3000$ & (Hernández et al., 2004) \\
\hline
\end{tabular}

\section{II.1.2.10 TUNEL assay for apoptosis detection in Drosophila tissues}

TUNEL, or terminal deoxynucleotidyl transferase-mediated düTP nick endlabeling, is a method of choice for rapid identification and quantification of the apoptotic cell fraction in cultured-cell preparations and tissues. Identification of the low molecular weight DNA fragments as well as single strand breaks ("nicks") in high molecular weight DNA can be carried out by labeling free 3'-OH termini with modified nucleotides in an enzymatic reaction. Terminal deoxynucleotidyl transferase (TdT), which catalyzes polymerization of nucleotides to free 3'-OH DNA ends in a template-independent manner, is used to label DNA strand breaks. Incorporated nucleotides are detected by a secondary antibody, conjugated with peroxidase. After substrate reaction, stained cells can be detected under light microscope. In situ cell death detection kit, AP (Alkaline Phosphatase) was used to detect apoptosis (Boheringer).

Protocol for TUNEL to detect apoptosis in Drosophila tissues:

- Dissect larval tissues in PBS for not more than 30 minutes

- Fix with $4 \%$ formaldehyde in PBS for 30 minutes at RT

- Permeabilize in $1 \%$ Triton in PBS for 15 minutes at RT

- Wash with $50 \mu \mathrm{L}$ Buffer 2 of the TUNEL kit

- Incubate 2 hours at $30^{\circ} \mathrm{C}$ with $45 \mu \mathrm{L}$ Buffer 2 and $5 \mu \mathrm{L}$ Enzyme TT of the kit 
- Wash $3 \mathrm{x}$ for 30 minutes with PBT

- Incubate tissues with 0.7:1000 AP-coupled anti-fluorescein-AP Fab fragment (Boheringer) overnight at $4^{\circ} \mathrm{C}$

- Wash $3 \times$ for 30 minutes with PBT

- Rinse with AP Buffer

- Develop with $1 \mathrm{~mL}$ BCIP/NBT solution (3.5 $\mu \mathrm{L}$ BCIP/4.5 $\mu \mathrm{L}$ NBT in $1 \mathrm{~mL}$ AP-Buffer $\mathrm{pH}$ 9.5)

- Wash 3 x for 30 minutes with PBT

- Mount tissues in Mowiol 4-88 (Calbiochem) and store at $4^{\circ} \mathrm{C}$ in the dark

- Visualize apoptosis with a phase contrast microscope

\section{II.2 Drosophila genetics methods}

\section{II.2.1 Generation of transgenic fly lines}

DNA sequences: Pc promoter:phGFP gene and esc promoter:escGFP gene sequences were inserted into the pUAST vector (Brand and Perrimon, 1993). Transgenic lines containing the construct P[Promoter:Gene] were generated with standard transformation protocols using the $w^{1118}$ host line (Spradling and Rubin, 1982) and the site of chromosome integration was determined genetically.

\section{II.2.2 Fly strais and culture}

The following strains were used in this study:

1. $w^{1118} ; P\left\{p P c-P c G F P, w^{+}\right\}$;

2. $w^{1118} ; P\left\{U A S, P c-P h G F P, w^{+}\right\}$;

3. $y w ; P\{e n 2.4-G A L 4\} e 22 c / S M 5$ (to drive expression of phGFP in embryos) 
4. $P\left\{G A L 4 ; w^{+}\right\} B x^{M S 1096}$ (to drive expression of phGFP in wing imaginal discs) where it drives the expression of Gal4 in the whole wing blade (Capdevila and Guerrero, 1994).

5. $p h^{504} w^{-}\left[F R T 101 w^{+}\right] / F M 7 C w^{a} B$

6. $p 53^{5 A-1-4}$ (Rong et al., 2002)

All strains were maintained on standard corn-agar medium at $18^{\circ} \mathrm{C}$ and experiments were done at $25^{\circ} \mathrm{C}$. The $p c G F P$ stock was kindly provided by R. Paro (Dietzel et al., 1999) and the en:GAL4 and $B x^{M S 1096}: G A L 4$ (Milan et al., 1998) drivers were provided by $\mathrm{H}$. Jäckle. 


\section{II.2.3 Attempt to rescue of apoptosis using a p53 mutant line}

Expression of phGFP under the control of the $P c$ promoter in salivary glands did not affect the development of the transgenic flies. But as soon as I used any kind of UASGAL4 controlled expression in any other somatic tissue at any developmental stage, apoptosis has been induced and as a consequence death of the animal (see Results for details).

Therefore, I wanted to see if apoptosis is induced through the p53 pathway and if it can be abolished in a p53 null background. The following crosses have been set up:

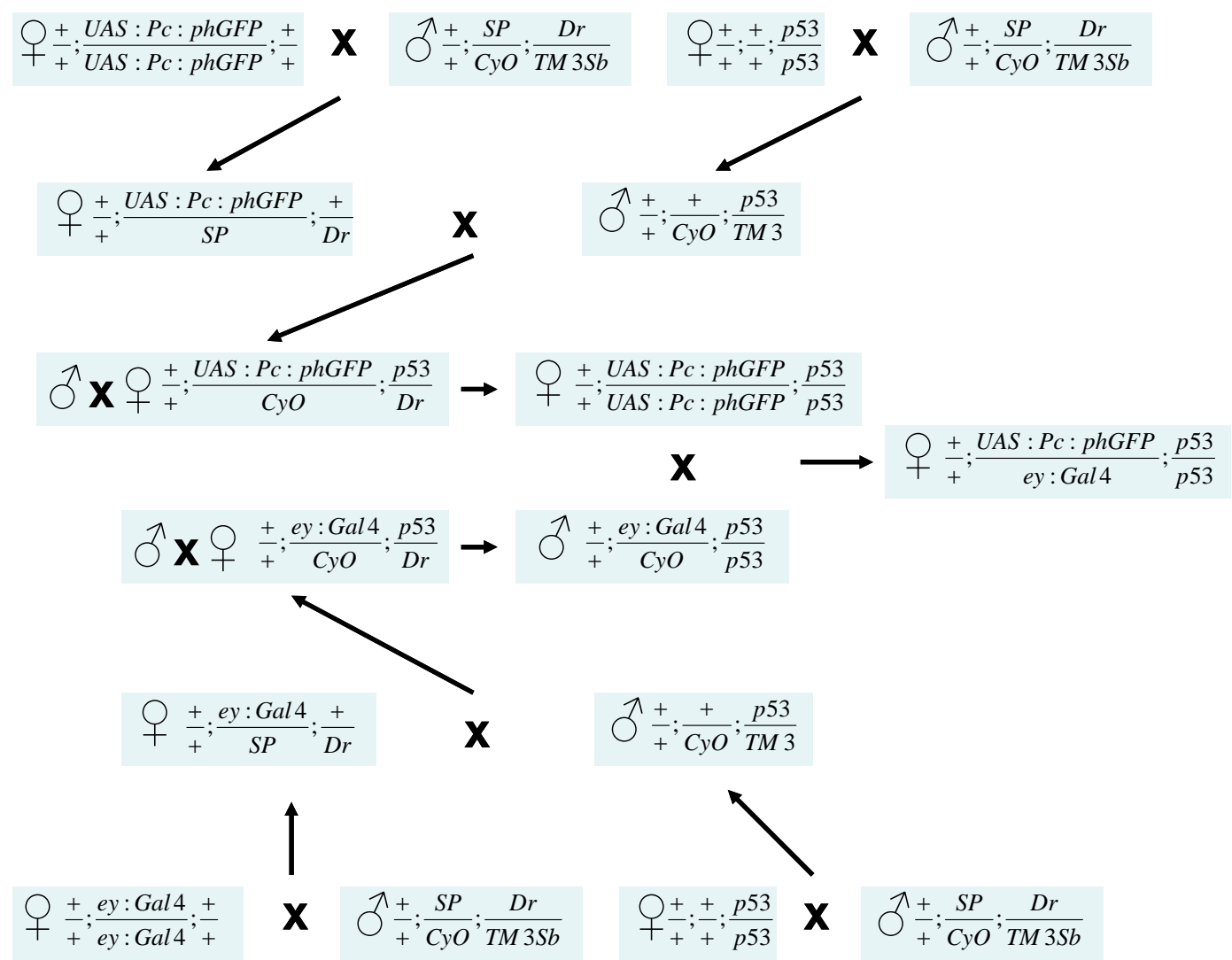




\section{II.2.4 Rescue of $p h$ null mutation phenotype by phGFP}

I set up the following crosses to see if my phGFP construct is able to rescue the $p h$ null phenotype (see description in the Results section):

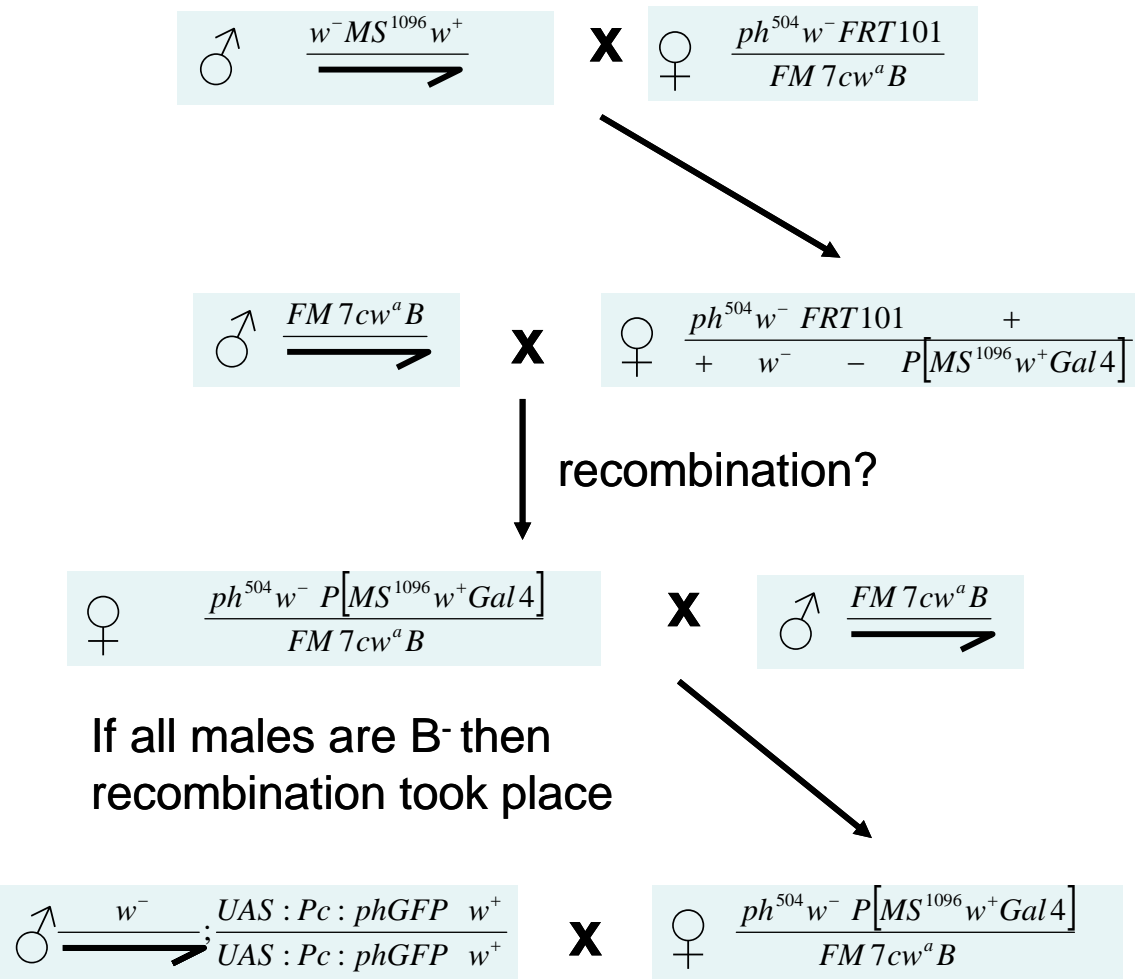

As a negative control cross I used:

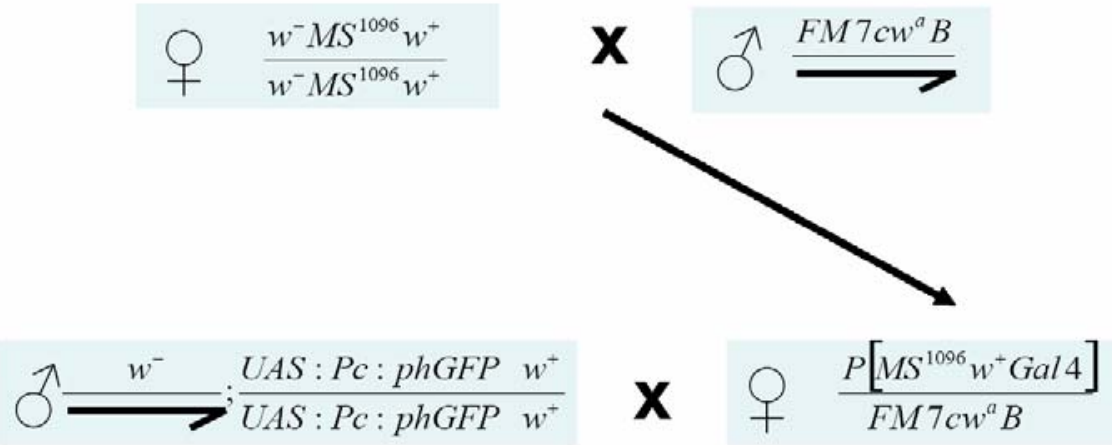




\section{II.3 Biophysical materials and methods}

\section{II.3.1 Materials}

\section{II.3.1.1 Phase contrast microscope}

To analyze the wing morphology from adult flies overexpressing PhGFP and the results from the TUNEL assay performed on wing and eye imaginal discs from L3 larvae overexpressing PhGFP in eye and wing discs, respectively, I used a Zeiss phase contrast microscope with a 2.5x/ 0.075 Plan-Neofluar objective. Wings of adult flies show necrotic patches revealed by the higher contrast regions and the apoptotic cells in the tissue are identified by the black patches as well.

\section{II.3.1.1.1 Mounting of specimens for microscopy and imaging}

Imaginal discs were mounted in Mowiol 4-88 (Calbiochem), covered with coverslips and sealed with nail polish. Mounting of adult fly wings was carried as following:

Materials: $\quad \mathrm{KOH} \mathrm{10 \% ,} \mathrm{Ethanol} \mathrm{abs.,} \mathrm{Lactic} \mathrm{acid,} \mathrm{nail} \mathrm{polish}$

SH solution: - 1 volume Glycerol

- 2 volumes Ethanol abs

(store flies here at RT)

- Put a drop of SH solution on a slide with depression; put flies in it

- Cut wings in water

- Wash wings $2 \mathrm{x}$ with water

- Wash wings $2 \mathrm{x}$ with ethanol abs

- Remove the ethanol abs

- add Lactic acid: ethanol 100\% = 6:5; mount wings in this mixture 


\section{II.3.1.2 Confocal scanning microscope}

Photobleaching experiments were performed with a Zeiss 510 Confocal Laser Scanning microscope with a C-Apochromat 63x/1.2 NA water corrected immersion objective. GFP was excited with the $488 \mathrm{~nm}$ line of an Argon ion laser and emission collected between 505 and $545 \mathrm{~nm}$ with a pinhole equivalent to 2 Airy discs.

\section{II.3.1.2.1 Mounting of specimens for microscopy and imaging}

For live imaging dechorionated embryos were transferred to a chamber with a coverslip bottom (LabTek) in oxygenated Tyrode's buffer (135 mM NaCl, $10 \mathrm{mM} \mathrm{KCl}$, $0.4 \mathrm{mM} \mathrm{MgCl}$, $1 \mathrm{mM} \mathrm{CaCl2,} 5.6 \mathrm{mM}$ glucose, $10 \mathrm{mM}$ HEPES, $\mathrm{pH}$ 7.2). In order to prevent movement and buffer evaporation they were covered with a polycarbonate membrane with $8 \mu \mathrm{m}$ pores that allowed oxygen exchange (Nucleopore, Pleasanton, CA). Larval imaginal and salivary gland tissues were dissected in PBS and immediately transferred to similar chambers and covered with a Whatman 3M filter paper soaked in Tyrode’s buffer. Imaging was performed at $21^{\circ} \mathrm{C}$ for a maximum of 2 hours after mounting.

\section{II.3.1.3 Software}

- Zeiss LSM 510 software for database and image handling

- Khoros/Cantata

Khoros and its user interface Cantata is a suite of programs for multidimensional image processing. The system further allows for relatively simple integration of user supplied image processing routines, even including the creation of a graphical user interface. For the scope of this thesis the standard Khoros 2000 system was used, which used to be freely available on the web. In addition several routines by Dr. Rainer Heintzmann, which were incorporated into Cantata, were used.

- Matlab/DIP image

Matlab and the Matlab toolbox DIPImage from Delft University (available at http://www.ph.tn.tudelft.nl/DIPlib) are very powerful suite of image processing routines. 
For the detailed simulations of the diffusion and binding with boundary values these formed the platform on which the simulation software was developed. Also the analysis of the experimental data was mostly based on Matlab/DIPImage routines.

- View5D

View5D (http://www.gwdg.de/ rheintz/View5D/) is a graphical front-end for the display and analysis of up to five dimensional datasets. In the analysis of the iFRAP data its particle-tracking features were used to determine the alignment parameters for successive image registration and analysis in Khoros/Cantata.

\section{II.3.2 Methods}

\section{II.3.2.1 Photobleaching methods}

FRAP images in somatic cell nuclei were performed with an XY sampling of $0.10 \mu \mathrm{m} /$ pixel and in polyploid salivary gland nuclei, at $0.14 \mu \mathrm{m} /$ pixel. Photobleaching was carried out for $\sim 200 \mathrm{~ms}$ (FRAP in salivary gland nuclei) at $\sim 200 \mu \mathrm{W}$ laser power (measured through the objective). Pre-bleach and post-bleach images were acquired at high scanning speed with minimal laser intensity (AOTF $2 \%, \sim 5 \mu \mathrm{W}$ ). At later measurement times (after frame 20), the interval between scans was increased in order to reduce bleaching during monitoring. 


\section{II.3.2.1.1 FRAP (fluorescence recovery after photobleaching)}

In FRAP a region of interest is shortly $(<200 \mathrm{~ms}$ ) bleached at a high laser power that will lead to photodestruction of the fluorescent molecules in that area. Recovery of the fluorescence in the bleached are is then monitored at the lowest possible laser intensity to avoid monitoring photobleaching. This method is suitable for biological samples where no major movement of the cytoplasmic or nuclear contents occurs.
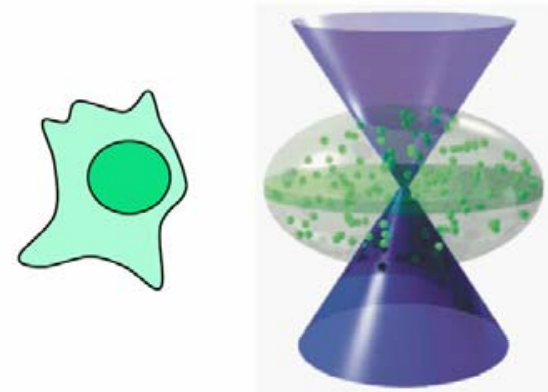

Prebleach
Photobleaching

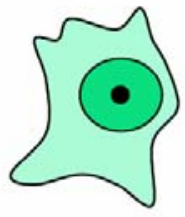

$100 \%$ immobile

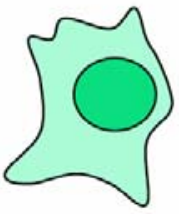

$0 \%$ immobile

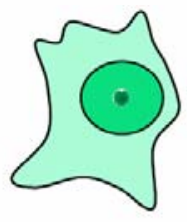

Postbleach

$33 \%$ immobile
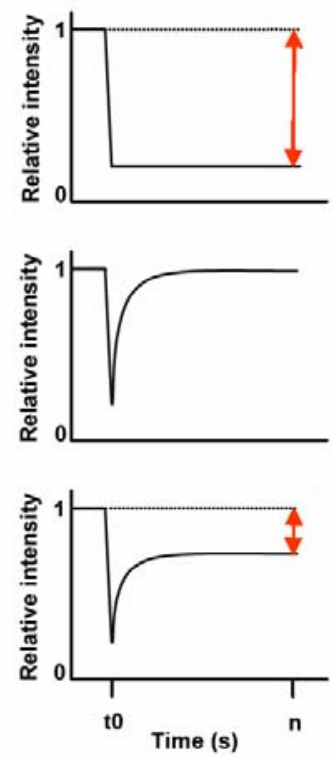

Figure 6. Fluorescence recovery after photobleaching (FRAP). A small region of interest (ROI) is subjected to a short bleach pulse at high laser intensity. The fluorescent intensity is subsequently monitored at low laser intensity. 


\section{II.3.2.1.2 iFRAP (inverse FRAP)}

iFRAP or inverse FRAP is a photobleaching method where the whole nucleus or cell is bleached except a region if interest (Fig. 7). Decay of the fluorescence in this region is monitored over time.

A variation of iFRAP that I introduced as a photobleaching method is 3D-iFRAP (three dimensional inverse FRAP). 3D-iFRAP experiments in embryos and imaginal discs were carried out by bleaching the whole nucleus except for a small region surrounding a fluorescent locus of interest for $\sim 4$ seconds. A time series of 7 confocal $\mathrm{z}$ sections (with 5 second intervals between stacks for PhGFP and 10 seconds for PcGFP) were recorded for 120 seconds after bleaching.
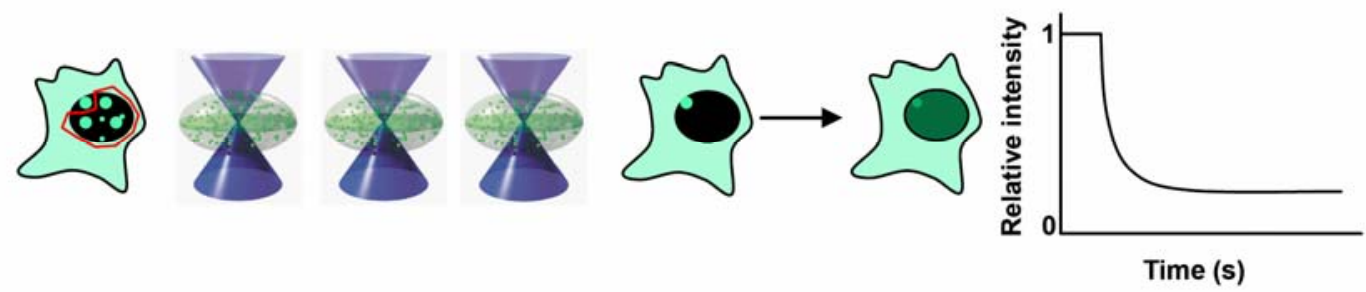

Figure 7. Inverse FRAP (iFRAP) and three dimensional iFRAP (3D-iFRAP). The whole cell or nucleus except a region of interest is subjected to a longer bleach pulse at high laser intensity until the fluorescence in the nucleus is depleted. The fluorescent intensity decay in the region of interest is then monitored at low laser intensity. In 3DiFRAP fluorescence decay is recorded in three dimensions by applying a series of $\mathrm{z}$ stack in contrary to the classical iFRAP where only one focal plane is used.

\section{II.3.2.1.3 FLIP (fluorescence loss in photobleaching)}

Another method for monitoring loss of fluorescence from a region in the nucleus is FLIP (fluorescence loss in photobleaching, Fig. 8). In this case a different region than the region of interest is subjected to pulses of high laser power. Between two pulses an image of the entire nucleus is taken at low laser power. Fluorescence exchange between the region of interest and the rest of the nucleus is measured over time. In my experience this is not a good choice for Drosophila nuclei (see Results). 

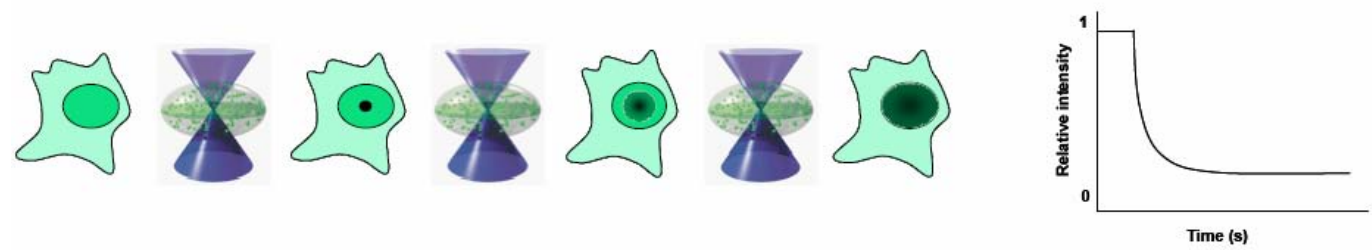

Figure 8. Fluorescence Loss In Photobleaching (FLIP). A small region of interest (ROI) is alternatively photobleached (a short bleach pulse at a high laser power) and monitored (at a low laser power). The process is repeated until all mobile fluorescent molecules are depleted.

\section{II.3.2.1.4 Half-FRAP}

Half-FRAP can be used to analyze a general fluorescence exchange between regions in the nucleus. As shown in Fig. 9 half of the nucleus is bleached and after the bleaching, both halves can be monitored for the fluorescence exchange.
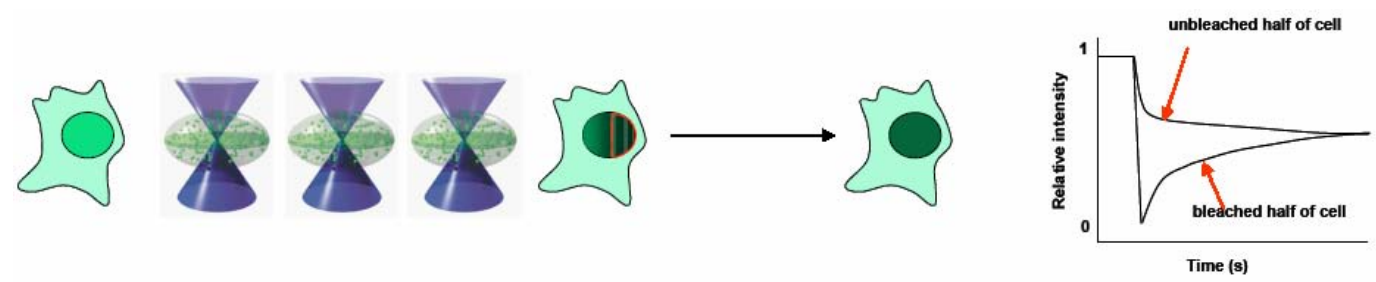

Figure 9. Half-FRAP. Half of the nucleus is subjected to a longer bleach pulse at high laser intensity. The fluorescence increase in the bleached region and the decay in the other half are then monitored at low laser intensity as illustrated by the curves on the right side.

\section{II.3.2.2 Image processing and fitting algorithms}

Background fluorescence in all photobleaching experiments was measured in a user-defined field outside the tissue for each experiment separately or estimated directly from the acquired image. An average loss of fluorescence intensity during imaging was corrected for in the evaluation of FRAP and iFRAP data via normalization to timedependent average intensity plots from separate nuclei imaged under identical conditions to the FRAP and iFRAP experiments. This correction was always $<10 \%$. The relative 
increase (FRAP) or decrease (iFRAP) in fluorescence intensity, corrected for background and bleaching during recovery, was normalized to the pre-bleach value and these $I_{\text {norm }}$ values were plotted for each time point:

$$
I_{\text {norm }}=\frac{I_{i}-B G}{I_{0}-B G} \cdot \frac{I_{0}^{r e f}-B G}{I_{i}^{r e f}-B G}
$$

where BG is the background intensity, $I_{i}$ is the average intensity of the ROI in image $i, I_{0}$ is the pre-bleach intensity in the ROI, $I_{0}^{\text {ref }}$ is the pre-bleach average intensity of an unbleached reference cell and ${ }^{r e f}$ is the intensity of the reference cell at image $i$. In the salivary gland nuclei, the total photobleaching during monitoring was $<5 \%$, obviating the need for a bleach correction during recovery. Images were corrected for XY drift by crosscorrelation prior to quantification. In the 3D-iFRAP experiments, the spot intensity was calculated in 3 dimensions using a weighted region of interest after an alignment based on a tracking algorithm (using the View5D plugin to ImageJ developed in this lab, http://www.gwdg.de/ rheintz/View5D/). The half-maximum recovery time $\left(t_{0}\right)$ in FRAP in preblastoderm embryos, the time required for the fluorescence intensity to recover half way between the first post-bleach level and the final height of the recovery curve, was determined by fitting the recovery curves to the following function:

$$
y=a+b \cdot \frac{t}{t+t_{0}}
$$

where $t_{0}$ corresponds to the half-maximum recovery time, $a$ is the offset of the curve and $b$ is the amplitude of the recovery curve. The diffusion constant was calculated using the translational diffusion equation described by Axelrod et al. (Axelrod et al., 1976):

$$
D=\frac{w^{2}}{4 \cdot t_{0}} \cdot \gamma_{D}
$$

where $w$ is the radius of the bleach spot $(\mu \mathrm{m}), t_{0}$ is the half maximum recovery time (s) and $\gamma_{D}$ is the correction factor for the shape of the bleaching beam. Using computer simulations I calculated the correction factor taking into account the diameter of the nucleus (8 $\mu \mathrm{m}$ for the preblastoderm nucleus and $25 \mu \mathrm{m}$ for the salivary gland nucleus) 
with the half-width of the bleach box as $w(0.75 \mu \mathrm{m}$ for the preblastoderm nucleus and 1.75 $\mu \mathrm{m}$ for the salivary gland nucleus) which yielded a $\gamma_{D}$ of 0.97 and 1.03 for the preblastoderm nuclei and salivary gland nuclei, respectively.

In FRAP experiments on bands of PcG proteins in salivary gland nuclei the free fraction was estimated by averaging the intensity in each frame near the spot in a region as defined by the lowest 30\% voxels of the sum intensity projection over all aligned prebleach frames (see Fig. 30). This nucleoplasmic signal of the free protein estimated frame by frame was subtracted from each pixel and the total bound protein was determined as the sum of all pixels in the mask region of the $30 \%$ brightest pixels in the projection over all aligned pre-bleach frames.

Recovery curves were fitted with a single exponential function after excluding the first 30 seconds after bleaching, during which diffusion still influences the data in spite of the correction for free protein:

$$
y=a+b \cdot\left(1-e^{-t / t_{0}}\right)
$$

where $t_{0}$ is the time required for the fluorescence intensity to reach $\sim 63 \%$ of the final height of the recovery curve for the bound molecules, $a$ is the offset of the curve and $b$ is the amplitude of the recovery curve. According to the grid-based simulations the influence of a spatially extended area of binding sites influences the binding kinetics in combination with the diffusion. The simulations also showed that there is only a minor influence of the size of a spot on its recovery kinetics as long as the total number of binding sites does not change. In other words, if an intense spot is doubled in size but has only half the concentration of binding sites, its recovery kinetics remains very similar. Also if the number of binding sites is doubled along with a doubling of the concentration of free molecules, the kinetics does not change. Since the measured spots all have different sizes and intensities, I correct the measured single exponential recoveries according to the model. I define

$$
B_{\text {ratio }}=\frac{I_{\text {bound }}-I_{\text {nuc }}}{I_{\text {nuc }}} \cdot P_{\text {bound }}
$$

where $B_{\text {ratio }}$ is the ratio of total bound protein in the locus to the average free protein where $I_{\text {bound }}$ is the mean intensity of the $30 \%$ highest pixels in the ROI, $I_{n u c}$ is the mean 
intensity of $30 \%$ lowest pixels in the ROI (free protein in the nucleoplasm) and $P_{\text {bound }}$ is the number of pixels in the mask region of the bound molecules.

Simulations showed an approximately linear dependence of the $t_{0}$ values to the $B_{\text {ratio }}$ of a locus using different fixed dissociation rate constants and a diffusion constant derived from the experimental data $\left(D=0.5 \mu \mathrm{m}^{2} \mathrm{~s}^{-1}\right)$ as is shown by the lines in Fig. 26. The recovery times $\left(t_{0}\right)$ for the measured data were corrected for this dependence according to the approximation (6).

$$
t_{0}^{*}=t_{0}-\frac{B_{\text {ratio }}}{1.23 \mu m^{2}}
$$

where $t_{0}$ is the experimental FRAP recovery time for individual loci as shown in Fig. 31B and $1.23 \mu^{2}$ is the slope of the simulation curves in Fig. 31B. The resulting dissociation rate constants for each individual locus are derived from the equality $k_{\text {off }}=1 / t_{0}{ }^{*}$. These values are plotted in Fig. 32 against the $B_{\text {ratio }}$ normalized by equating the highest ratio to $1 \mu \mathrm{m}^{2}$ whereby there is no obvious correlation of the dissociation rate constants to the number of binding sites in a locus.

The pseudo reassociation rate constant $k_{\text {on }}{ }^{*}$ was calculated according to the method described in Sprague et al. 2004 that defines a pseudo-first-order rate constant given by

$$
\begin{array}{r}
k_{\text {on }}^{*}=k_{\text {off }} \cdot \frac{C_{e q}}{F_{\text {eq }}} \\
k_{\text {on }}=\frac{k_{\text {on }}^{*}}{C_{s}}
\end{array}
$$

where $k_{o n}{ }^{*}$ is the pseudo first order association rate constant, $k_{o n}$ is the second order association rate constant, $C_{s}$ is the unknown concentration of binding sites, $C_{e q}$ is the concentration of bound protein at equilibrium (mean intensity of the bound fraction) and $F_{\text {eq }}$ is the concentration of the free protein at equilibrium (mean intensity in the nucleoplasm). 


\section{II.3.2.3 Simulation methods: differential equations for the binding and dissociation of fluorescent and non-fluorescent molecules to fixed binding sites}

\section{II.3.2.3.1 Assumptions}

There are dark (d-non-fluorescent) and bright (b-fluorescent) species of the same molecules which compete for spatially and temporally fixed binding sites with a spatial concentration $c_{s}(x)$. A bound molecule is indicated with a subscript $a$ for attached, where free molecules are indicated by subscript $f$. There is equilibrium between binding and dissociation of all molecules. The attached molecules cannot diffuse as the binding sites are fixed.

There are two rate constants involved $\mathrm{k}_{\text {on }}\left[\frac{1}{\text { time }} \cdot \frac{\text { Volume }}{\text { Number }}\right]$, the rate for binding to a site which is concentration dependent (second order) and $\mathrm{k}_{\text {off }}\left[\frac{1}{\text { time }}\right]$, the rate constant to unbind, which is first order. These constants are assumed to be equivalent for nonfluorescent and fluorescent molecules. This means that the process of photodestruction is assumed to not alter the binding kinetics of a molecule. The diffusion constant $\mathrm{D}$ is constant in time and space.

The measurement gives the intensity $I(x, t)=b_{f}(x, t)+b_{a}(x, t)$.

$b_{f} \quad$ bright free (fluorescent free)

$b_{a} \quad$ bright attached (fluorescent attached)

$d_{f} \quad$ dark free (non-fluorescent free)

$d_{a} \quad$ dark attached (non-fluorescent attached) 


\section{II.3.2.3.2 Equations}

The probability that binding sites at a specific location are free is $P_{s}=1-\frac{d_{a}+b_{a}}{c_{s}}$.

The local total concentration of binding site is denoted by $c_{s}$. We obtain the following equations for the fluorescent molecule state

$$
\begin{aligned}
& \partial_{t} b_{f}=-D \Delta b_{f}+k_{\text {off }} b_{a}-k_{\text {on }} b_{f} P_{s} c_{s} \\
& \partial_{t} b_{a}=-k_{\text {off }} b_{a}+k_{\text {on }} b_{f} P_{s} c_{s}
\end{aligned}
$$

and for the non-fluorescent state

$$
\begin{aligned}
& \partial_{t} d_{f}=-D \Delta d_{f}+k_{\text {off }} d_{a}-k_{\text {on }} d_{f} P_{s} c_{s} \\
& \partial_{t} d_{a}=-k_{\text {off }} d_{a}+k_{\text {on }} d_{f} P_{s} c_{s}
\end{aligned}
$$

Here $\partial_{t}$ denotes differentiation with respect to time, $\Delta$ is the Laplace operator (sum of second spatial derivatives)

These four equations decouple in separate systems for non-fluorescent and fluorescent state if the probability that a site is free is constant in time $P_{s}=$ const., i.e. if the binding and dissociation is in equilibrium $d_{a}(x, t)+b_{a}(x, t)=$ const. $(x)$. The introduction of $C_{s}(X)$ and $P_{s}$ allows to split the binding probability into a spatial part by the distribution of $c_{s}(x)$ and a temporal part $P_{s}$, which is constant due to the equilibrium assumption here.

\section{II.3.2.3.3 Boundary conditions and solutions}

Even though the simulation is not restricted to a specific structure of $c_{s}$, binding sites, with their distribution given by $c_{s}(x)$, are localized in one or multiple small confined area (nucleus or part of it) for one experiment.

\section{II.3.2.3.4 Simulations}

We want to solve the differential equation in multiple dimensions. I use the alternating direction implicit method (ADI), which is unconditionally stable and second- 
order accurate in space and time and uses operator splitting (Press et al., Numerical Recepies in $\mathrm{C}++^{+}$, section 19.3). This is method is reasonably fast and stable.

The binding and dissociation is integrated in the standard diffusion as follows from eq.(1.3) and making the equation dimensionless:

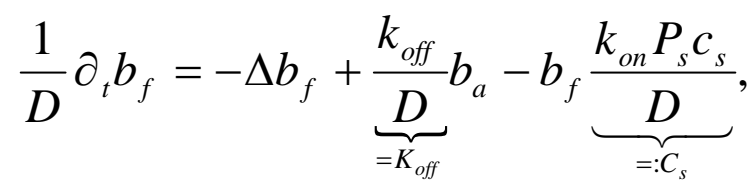

where the diffusion constant $\mathrm{D}$ is integrated in the discretisation of the time derivative on the left hand side. Eq.(1.4) is evaluated in between two full iteration steps as:

$$
\frac{1}{D} \partial_{t} b_{a}=-K_{\text {off }} b_{a}+C_{s} b_{f} .
$$

Thus for equilibrium we have

$$
\frac{b_{a}^{e q}(x)}{b_{f}^{e q}(x)}=\frac{C_{s}(x)}{K_{o f f}}=\frac{k_{o n} P_{s} c_{s}(x)}{k_{o f f}} .
$$

The ratio is to be computed for the distribution field, i.e. per pixel for the intensity images $b_{a}$ and $b_{f}$.

\section{II.3.2.3.5 Grid-based simulations}

Simulating spatially localized structures with multiple specific, well defined binding locations as opposed to the situation of diffusion in a homogeneous medium without boundaries usually requires a grid-based simulation approach. In principle a set of basis functions adopted to the model (like sine-waves) could be chosen. However, in this case it is often problematic to incorporate the boundary conditions (no diffusion allowed across the nuclear membrane). Even though multi-resolution grid approaches would be an option, I opted to simulate the set of differential equations on a regular square-grid to keep it simple. The differential equations in a discrete domain (steps in time and space) connect 
differences in time (between the identical grid-point at consecutive iterations of the simulation) with differences in space (between neighbouring grid-points).

A straight forward simulation, such as calculating the spatial derivatives at one iteration step for each grid-point, to predict the time derivative and thus the value at this very grid-point in the next iteration turns out to be numerically instable (Press et al., Numerical Recepies in $\mathrm{C}++$ ). Instable means that small numerical errors (e.g. a spatial ripple) gets amplified in every iteration step and then quickly dominates the simulation result rendering it senseless. Therefore a stable updating scheme had to be selected, which is suited for the simulation of this (diffusion-) type of differential equations in multiple dimensions. The updating scheme that was selected is called "Alternating Directions Implicit in Time” (ADI-scheme). This means, that one full iteration is subdivided into two sub-iterations (A and B), each of them considering the spatial derivatives only along one of the directions (here two) in space. Implicit in time means that the spatial derivatives (and the time derivative) at the unknown (i+0.5) iteration are all linearly connected with the known values at step i. In one half-step each row of grid points inside the nucleus is implicitly updated using the column spatial derivatives and in the next half-step an analogous procedure is used for the rows. This situation requires solving a linear equation system (which can be written in matrix form) in each half-step for line (A) or column (B) of the simulation-grid in the respective sub iteration steps (A, B). The boundary conditions of von Neumann type restricting flux across the boundary can be worked into the equation systems. Solving the equation systems is straight forward and very fast, since the associated matrix is tri-diagonal.

To my knowledge, spatially localized binding and its interplay with diffusion of free binding partners has not been modelled up to date. In this study I will present detailed results of such simulations offering guidance in the interpretation of fluorescence recovery after photobleaching (FRAP) data in situations of spatially localized binding of freely diffusing proteins.

Even though a similar scheme could also be applied on a 3D grid, this has not been attempted. ANY initial situation of binding sites, bound proteins, spatially varying amount of bleaching, and even spatially varying diffusion constant can be simulated in this way.

Even though this simulation is two dimensional, it covers also a three dimensional situation in which all planes contain identical concentrations of all involved substances and boundary conditions ('cylindrical' symmetry). In this case, influx and outflux between the 
slices would always compensate rendering the model valid. I am aware of the fact that a spatially localized binding site typically is not present with equal concentration in all slices of the nucleus and bleaching with high-NA objective is not identically distributed in all slices (see below), which makes my model only an approximation to the real situation. I tried to correct for some of the effects by appropriate normalization of the experimental data prior to analysis. 


\section{RESULTS}

\section{III.1 Nuclear distribution and dynamics of PcG protein complexes in Drosophila development}

To investigate the in vivo properties of the Polycomb group of proteins, I have used transgenic Drosophila fly lines expressing the green fluorescent protein fused to members of the Polycomb repression complex. Developmental dynamics of Polycomb-GFP (PcGFP) has been described previously (Dietzel et al., 1999). In this study the subcellular distribution of PcGFP in early embryonic stages, salivary gland nuclei and in the male germ line has been described. Rapidly dividing nuclei have been found to display a homogeneous distribution of PcGFP whereas nuclei in later embryonic developmental stages and larval imaginal discs showed substructures with highly fluorescent spots. The construct has been shown to rescue alleles with a mutation in the Pc chromodomain $\left(\mathrm{Pc}^{\mathrm{XL} 5}\right)$ but not mutations in the C-terminal part or null mutations $\left(\mathrm{Pc}^{2}, \mathrm{Pc}^{3}\right)$. Another PcG protein, Polyhomeotic is present in the PRC1 repression complex in stoichiometric amounts with Polycomb and Posterior sex combs (Shao et al., 1999). A functional GAL4 driven PhGFP transgenic fly line was available (Netter et al., 2001) but the outcome of this strategy was dependent on the GAL4 driver employed, i.e. the polytene chromosomes showed a GFP signal above the basal level with a strong bulky pattern.

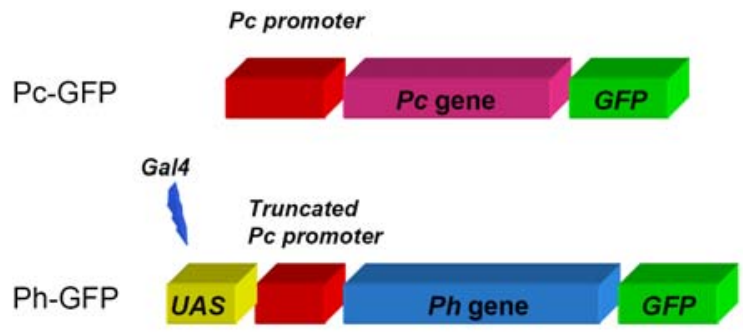

Figure 10. Constructs used to generate transgenic fly lines expressing PcG proteins fused to GFP. The PcGFP fly line was generated and described previously (Dietzel et al, 1999). The PhGFP construct was generated as part of this thesis. 
The salivary glands themselves were less than half the size of a wt salivary gland (data not shown). I therefore generated a construct for the PhGFP under the Polycomb promoter that shows a typical banded pattern in the salivary gland nuclei characteristic to PcG proteins. In a later chapter I describe the genetic crosses I have performed in order to assess the functionality of the protein. This construct was able to rescue the null mutant $p h^{405}$ (see section III. 8).

\section{III.1.1 Expression pattern of PcGFP and PhGFP in various developmental stages}

My primary objective was to determine rate constants for PcG protein complexes in living embryos and tissues of Drosophila during stages of development when the complexes are biologically competent. Whole mount embryos are viable and can proceed through the entire embryonic development process, i.e. through larval stages and pupation. Explanted imaginal discs cultured in vitro can develop and undergo metamorphosis by addition of hormones (Sengel and Mandaron, 1969). Thus both embryos and imaginal discs meet the criteria for living tissue. Genetic loci are locally represented in up to 1000 to 2000 copies in salivary glands, a differentiated tissue exclusive to larvae, and provide us with the possibility of observing and measuring the stability of complexes that bind to single or few PRE loci. Pc and Ph bind to an overlapping set of target genes on these polytene chromosomes (Franke et al., 1992). After the mid-blastula transition aggregates of PcG proteins appear in all 2N nuclei of the embryo.

The distribution of PhGFP and PcGFP in post-cellularization stages of embryogenesis and in wing imaginal discs is shown in Fig. 11. Although Pc promoter driven PhGFP expression is induced in the salivary gland nuclei, none of the various insertions of the PhGFP construct showed GFP signal above the background level in embryos and larval imaginal discs. Insertions on the $\mathrm{X}$ chromosome showed PhGFP expression in the nuclei of the peripodial cells of the wing discs but not in the somatic nuclei (data not shown). Therefore, in the embryos and larval diploid tissues, I had to induce PhGFP expression by crossing phGFP flies with various GAL4 driver lines. Driving of PhGFP expression in the embryos and larval wing imaginal discs was then achieved using the engrailed-GAL4 driver and the $B x^{M S 1096}$ :GAL4 driver, respectively. An 
embryo in stage 13 and a wing disc expressing PhGFP (with the striped engrailed pattern in embryos) and the corresponding nuclear expression pattern is shown in Fig. 11A, E and B, F, respectively. In the wt condition, Polycomb and Polyhomeotic are present in nuclei of all tissues throughout embryogenesis (Buchenau et al., 1998).

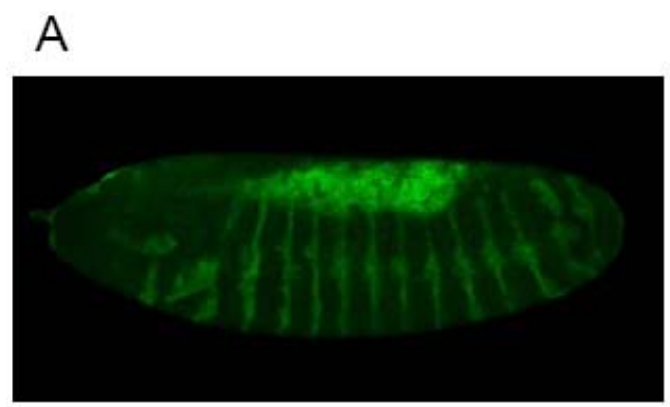

C

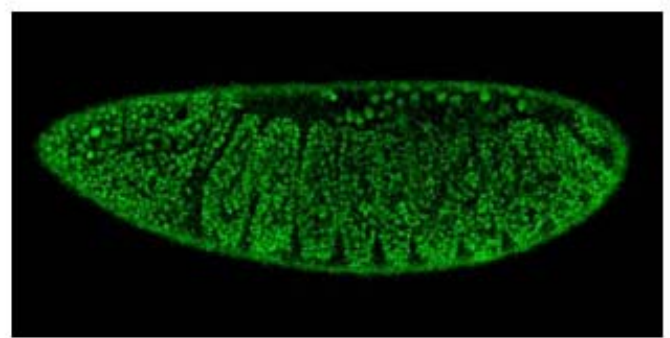

E

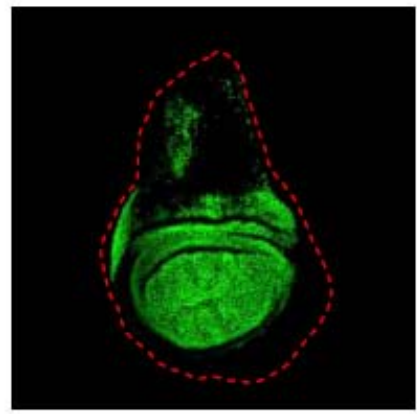

$$
\mathrm{H}
$$

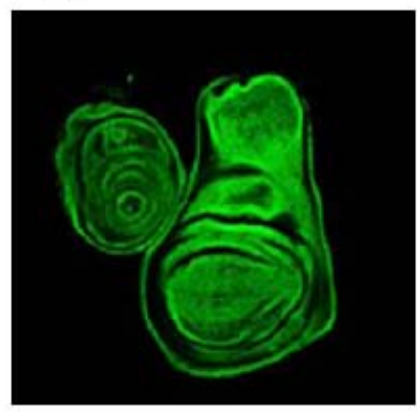

B

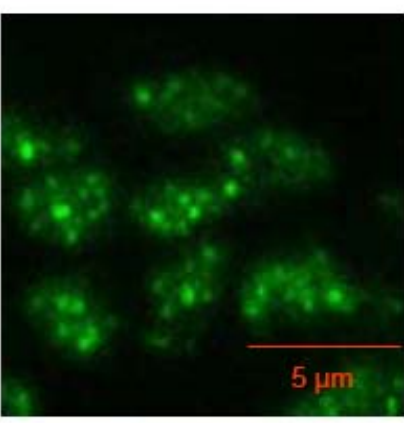

D

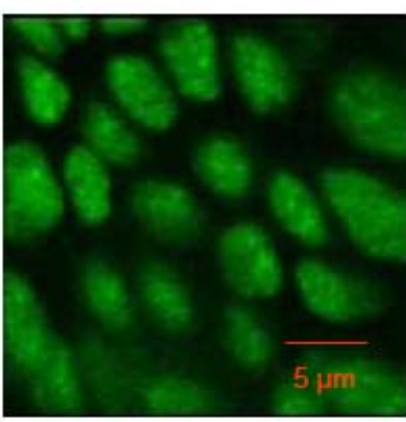

$\mathrm{F}$

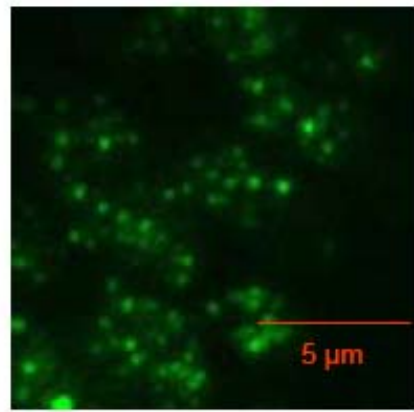

\section{I}

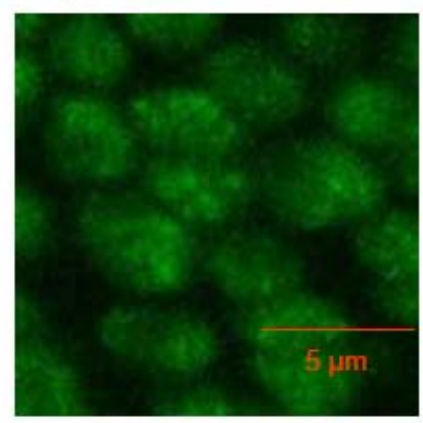



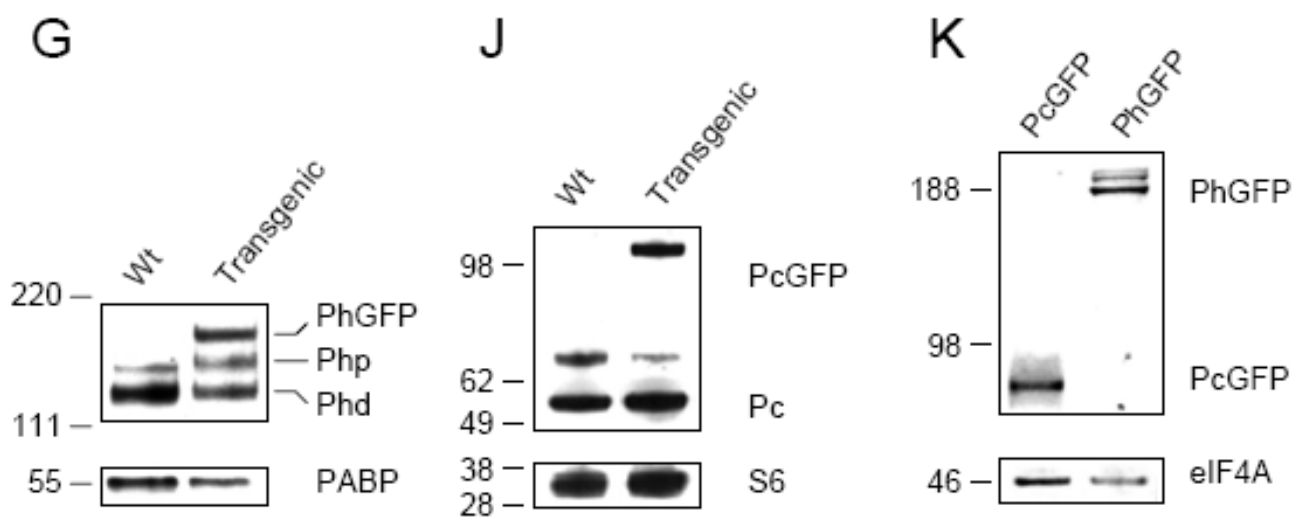

Figure 11. Distribution patterns of PhGFP and PcGFP in the embryos and larval wing discs. PhGFP expression was induced using the engrailed-GAL4 driver in embryos (A) and the $B x^{M S 1096}$ :GAL4 driver in wing imaginal discs (E). The corresponding distribution pattern of PhGFP in nuclei can be seen in (B) (embryos) and (F) (wing discs). PcGFP expression in embryos and wing imaginal discs is shown in $(\mathrm{C})$ and $(\mathrm{H})$ and the respective nuclei in (D) and (I). Western blot analysis of Drosophila tissue extracts from wildtype and transgenic fly lines in G, J and K. (G) Extracts from wing discs from wt and PhGFP expressing larvae (probed against Polyhomeotic). (J) Extracts from wt and PcGFP expressing embryos (probed against Polycomb). (K) Extracts from salivary glands from PcGFP and PhGFP expressing larvae (probed against GFP). Antibodies against Poly-A binding protein (PABP) $(\mathrm{G})$, S6 Ribosomal protein $(\mathrm{J})$ and eIF4A (K) were used for loading control.

Immunostaining of PcG proteins and 3D reconstruction of confocal images showed a spotted distribution of PcG proteins similar to the ones seen in Fig. 11. The number of such aggregates of $\mathrm{Ph}$ and $\mathrm{Pc}$ increases during embryogenesis such that by stage 14 greater than 100 such loci can be distinguished in fixed, whole mount embryos (a number similar to the number of bands observed on polytene chromosomes). The number of distinct loci in imaginal discs is an order of magnitude smaller than in embryos, suggesting that these loci are composed of higher-order aggregates (unpublished data of the authors in Buchenau et al, 1998). Imaging live cells of PcGFP and PhGFP expressing flies revealed a smaller number of spots but it is possible that the small amount of PcG proteins present in smaller loci cannot be detected as individual PcG loci due to decreased signal to noise ratio in live cell imaging. In addition PcGFP expression seems to be more diffuse throughout the nucleus in all tissues in contrast to PhGFP (Fig. 11 B,D and F,I). An explanation of this fact will be discussed in the following chapters. 
Table 3. Quantification of western blots in Fig. 2 G, $\mathbf{J}$ and $\mathrm{K}$

\begin{tabular}{|c|c|c|}
\hline Antibody/Normalized to: & Relative to: & Value \\
\hline \multirow[t]{3}{*}{ Polycomb/Pcwt } & $P c^{w t}$ & 1 \\
\hline & $P c^{\text {wt in transgene }}$ & 1.09 \\
\hline & PCGFP & 0.58 \\
\hline \multirow[t]{5}{*}{ Polyhomeotic/Phpwt } & $P h p^{w t}$ & 1 \\
\hline & $P h d^{w t}$ & 4.41 \\
\hline & Php wt in transgene & 2.45 \\
\hline & Phd wt in transgene & 3.26 \\
\hline & PhGFP & 3.42 \\
\hline \multirow[t]{2}{*}{ PhGFP/PcGFP } & PCGFP & 1 \\
\hline & PhGFP & 2.2 \\
\hline
\end{tabular}

Quantification of western blots of imaginal discs or embryos from wildtype and transgenic flies revealed slightly lower levels of GFP labeled proteins than the endogenous ones, such that the ratio of total Pc protein in transgenics (including PcGFP) was only 1.6 times that in wildtype and the ratio of total Ph (including PhGFP) in transgenics was 1.7 times that of wildtype (Fig. 11 G, J and Table 3). PhGFP expression in wing imaginal discs was induced by $B x^{M S 1096}: G A L 4$ driver that induces expression in the whole wing blade. The wing blade represents $60-70 \%$ of the wing disc. Therefore, the amount of PhGFP expressed per nucleus is comparable to the amount of total untagged $\mathrm{Ph}$ per nucleus. There was a change in the relative expression of the proximal to the distal $\mathrm{Ph}$ genes in the transgenic line as seen in Fig. 11G. The relative expression levels of PcGFP and PhGFP in transgenic salivary glands determined by Western blotting using an anti-GFP antibody were 1 to 2.2 (Fig. 11K). In the salivary gland nuclei both PcGFP and PhGFP expressions are induced by the Pc promoter (no GAL4 driver used in this case).

\section{III.1.2 Extensive mobility of PhGFP and PcGFP complexes in live gastrulating embryos and whole mount imaginal discs}

Live cell imaging is the method where spatial and temporal changes in the cellular environment can be followed over time. Imaging Drosophila embryos and larval tissues 
involves a higher level of complexity compared to cell cultures due to the continuous development of the organism. In a time span of a few hundreds of seconds I observed a significant directional movement of the nuclei (several cell diameters) and an extensive reorganization of the nuclear content.

Upon photobleaching the pool of free protein (by inverse FRAP or iFRAP) in a coupled system consisting of a free protein pool and stationary complexes (bound protein) with a substantially slower dissociation rate than the diffusion rate of the free protein, the rate of disappearance of the fluorescent locus will be a direct measure of the first order dissociation rate constant of the protein from the complex (Dundr et al., 2002). As shown in the following sections, the recovery times of the PcGFP and PhGFP complexes were at least an order of magnitude longer than recovery from free diffusion of the macromolecules. Thus, it was necessary to measure for times longer than 50 seconds in order to reach equilibrium between the redistribution of bleached and unbleached proteins. Nuclei and chromatin itself are not stationary in live Drosophila tissues, as shown in Figs. 12 and 13.

Core histone-GFP that does not dissociate from chromatin in interphase showed similar dynamics, indicating that the movement I see in my cells is not due to dissociation of whole complexes from the chromatin (Post et al., 2005). In addition, photobleaching of the nuclear lamin fused to RFP (red fluorescent protein) in embryonic and 2N larval disc Drosophila nuclei revealed no rotation of the nuclei over a period $>3$ minutes (personal communication, C. Fritsch). Therefore, it is the nuclear content that exhibits reorganization over time. It is necessary to mention that the amount of movement observed is not the same in all regions of the embryo and larval diploid tissues as well as in time after explanting the imaginal discs. Embryos undergo continuous development in the time-frame of $\sim 5$ minutes. There is mitosis taking place that will "push" the rest of the embryonic tissue. In imaginal discs cells undergo asynchronous cell division as well, therefore is it difficult to predict the amount of movement in various regions of the embryos and diploid larval tissues. Another observation is that the movement decreases 2-3 hours after mounting the tissue. This is due probably to the fact that the tissue is progressively dying out. 


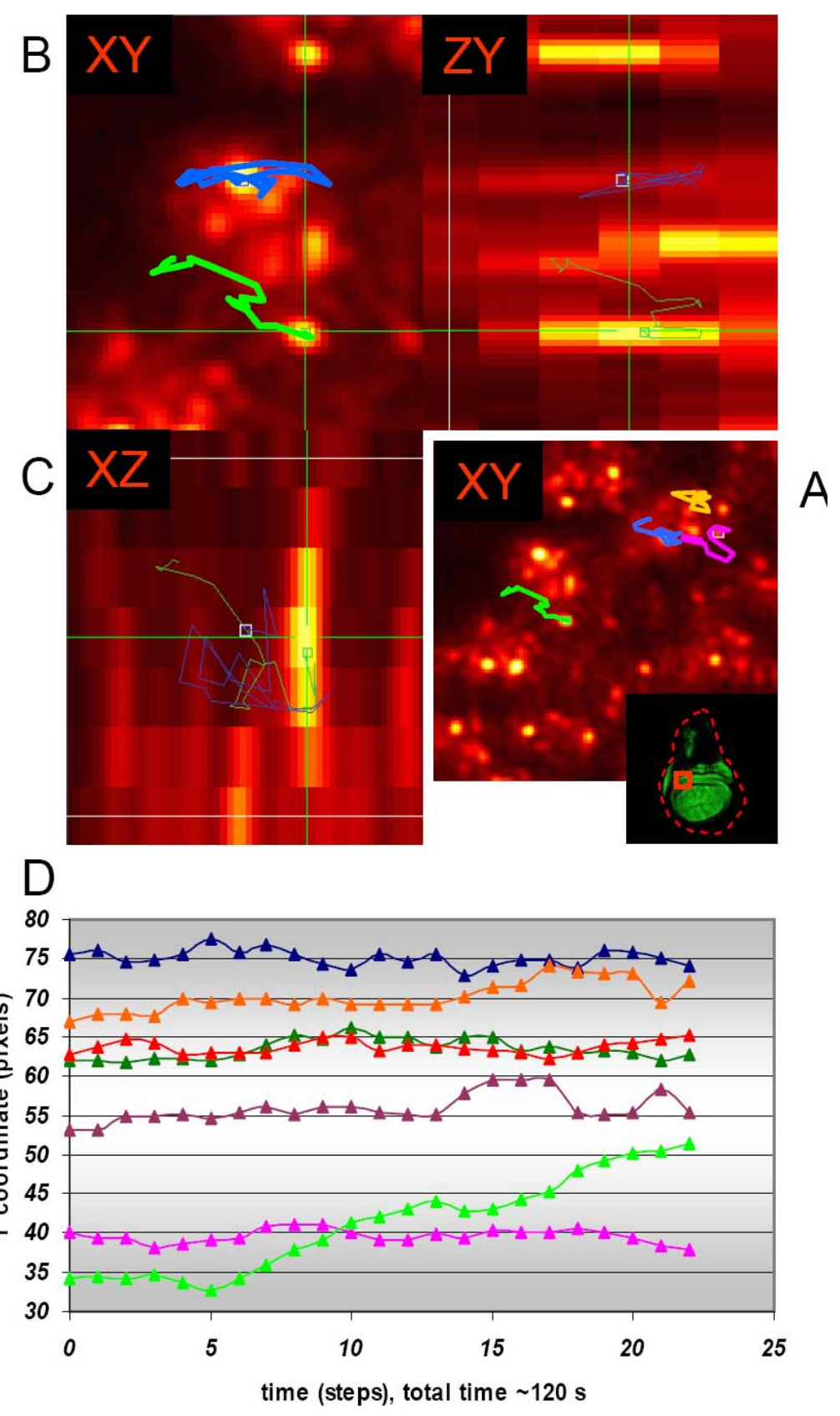

Figure 12. Chromatin dynamics in diploid nuclei. (A) Inset, fluorescence image of a PhGFP larval wing disc. Intensity pseudo-coloured magnified image of the region boxed in the inset. A stack of seven $\mathrm{Z}$ sections was imaged repeatedly at 5 second intervals for 120 seconds. Time traces of several loci are indicated by the coloured tracks. (B) A single $X Y$ plane at time 0 from the stacks is displayed. Time XY tracks are superimposed for 2 loci (blue and green traces). Zero time (C) XZ planes and (D) YZ planes for the slices designated by the cross-hair in image B. Time traces for the positions of the two loci are superimposed showing the large movements. 
Therefore it is important to acquire live processes possibly 20 minutes after the mounting, to allow recovery of the tissue from the explanting and mounting process, and in less than 2 hours after mounting to avoid artefacts in the measurements.

My observation was that embryonic nuclei exhibit a directional XYZ movement of groups of nuclei (probably due to mitosis that takes place in neighbouring regions) in addition to nuclear content reorganization whereas larval diploid tissues show a more random type of movement in various regions (Fig. 13). The most $\mathrm{Z}$ oriented movement has been seen in central region of the disc (regions 1 and 3, data not shown).

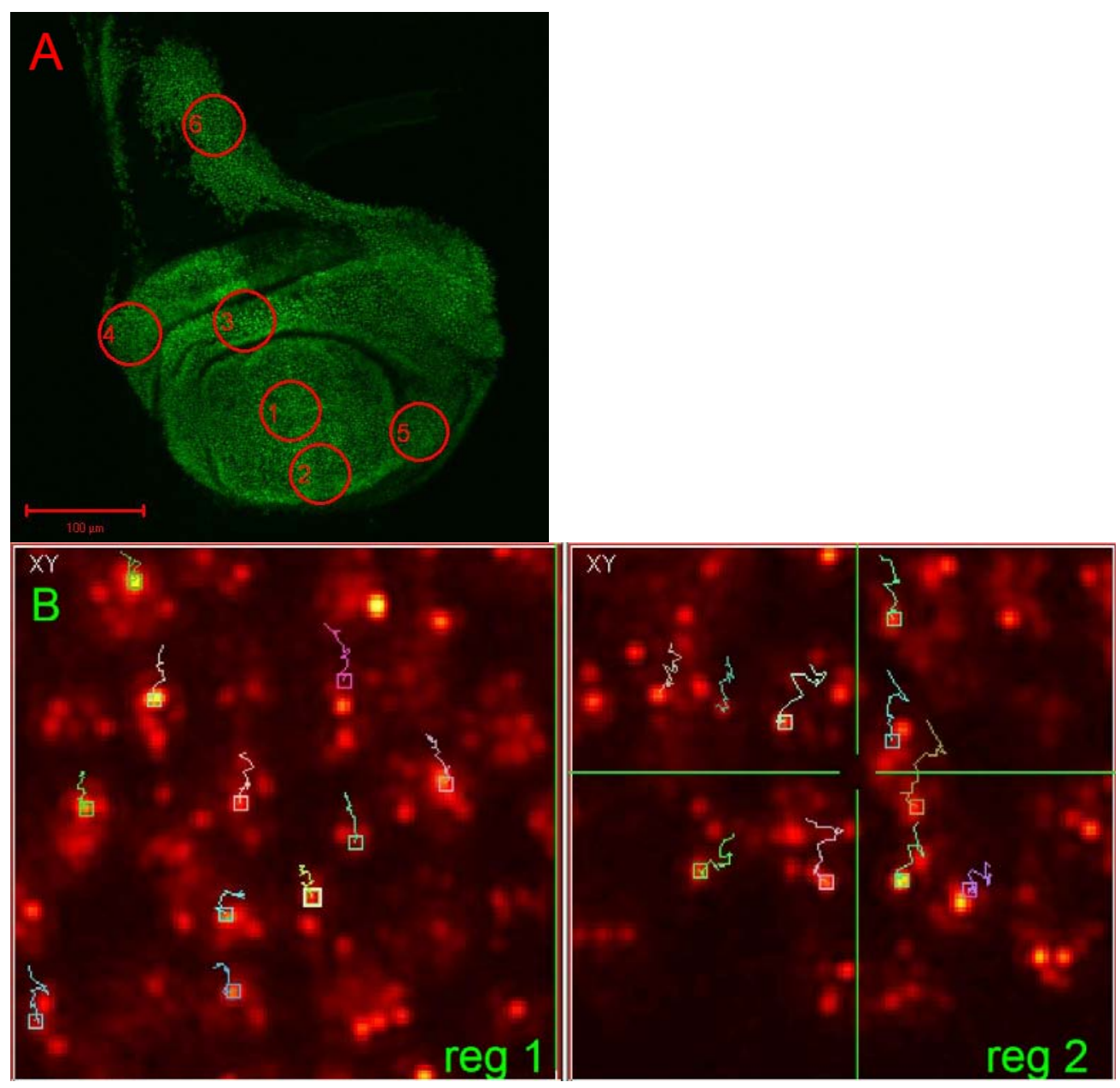

(continuation of the figure on the next page) 

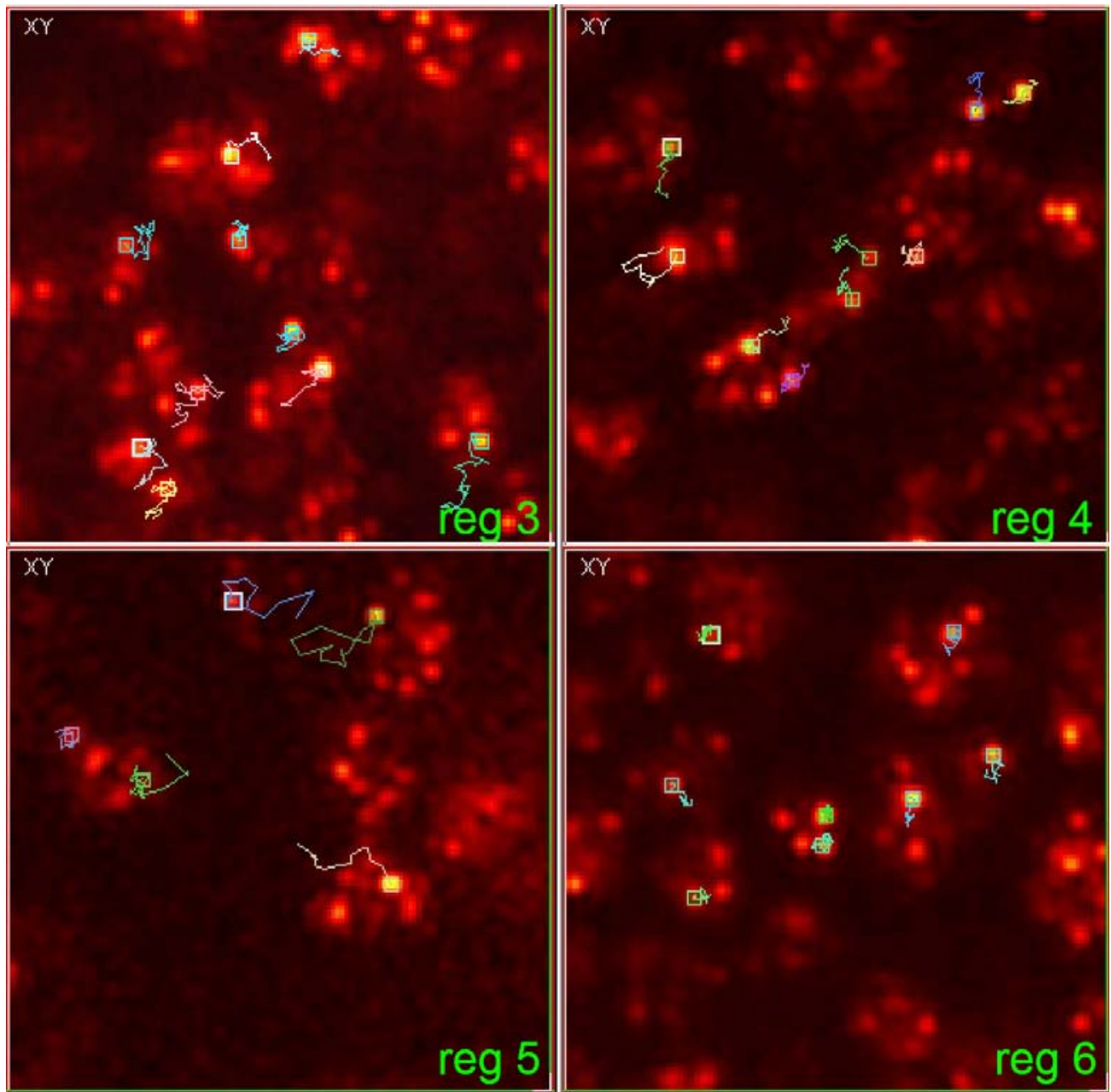

Figure 13. Chromatin movement in various regions of the wing imaginal discs. PcG loci in various regions (B) in the wing imaginal discs (A) have been tracked in four dimensions (20 zstacks - $5.7 \mu \mathrm{m}$ - for 330 seconds).

\section{III.2 Measuring diffusion constants of nuclear proteins}

Numerous cellular processes including metabolism, second messenger signaling and protein-protein interactions require diffusion of solutes and macromolecules in cellular compartments. Effects like fluid phase viscosity, transient binding and molecular crowding retard diffusion. Molecular size is also a strong determinant as it has been observed that the cytoplasmic "sieving” affects diffusion of bigger molecules much stronger than smaller ones where $\mathrm{D}_{\text {cyto }} / \mathrm{D}_{\text {water }}$ was only mildly altered (Luby-Phelps, 2000). Transient binding with higher or lower affinity can also severely restrict the mobility of molecules independent of their size. The diffusion of small and macromolecular solutes in cellular 
aqueous compartments is therefore determined by solute properties and by the composition, organization and geometry of the cellular compartment (Verkman, 2002). Although one would intuitively think that the high DNA content and the large amount of RNA and protein molecules would create a gel-like environment in the nucleus that would hinder rapid mobility of molecules, recently, biophysical measurements like FRAP and fluorescence correlation spectroscopy (FCS) have indicated unexpectedly high mobility of molecules in the nucleus (see Introduction).

\section{III.2.1 Diffusion of PcGFP and PhGFP in the Drosophila nuclei in embryos and larval tissues}

Before cellularization in Drosophila the PcG proteins are all of maternal origin and their binding to chromatin is restricted to a few PRE sites (Orlando et al., 1998). It is not clear if the repression complexes formed are functional since zygotic transcription has not yet begun. Thus, there exists a window in development (early division cycles) in which one can measure the diffusion of the fusion proteins by classical FRAP techniques. In the preblastoderm embryos the distribution of PcGFP is rather homogeneous throughout the nucleus (Dietzel et al., 1999) and the nuclear size is large relative to somatic diploid nuclei later in development (Fig. 14A). At cycle 10 I observed a few faint aggregates of PcGFP in a uniform fluorescent nucleoplasm. I measured the diffusion coefficient in regions without aggregations by conventional FRAP. The number and intensity of the PcGFP aggregated loci increases as embryonic development proceeds and as nuclei decrease in size. Another development stage providing access to the free protein is in the larval salivary gland nuclei where the chromatin is condensed in polytene chromosomes leaving regions of free nucleoplasm. 
A

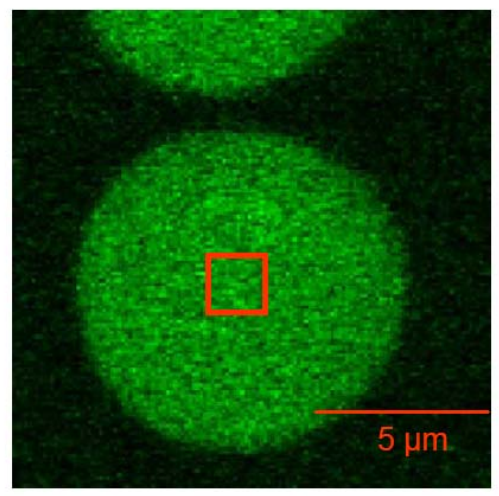

B

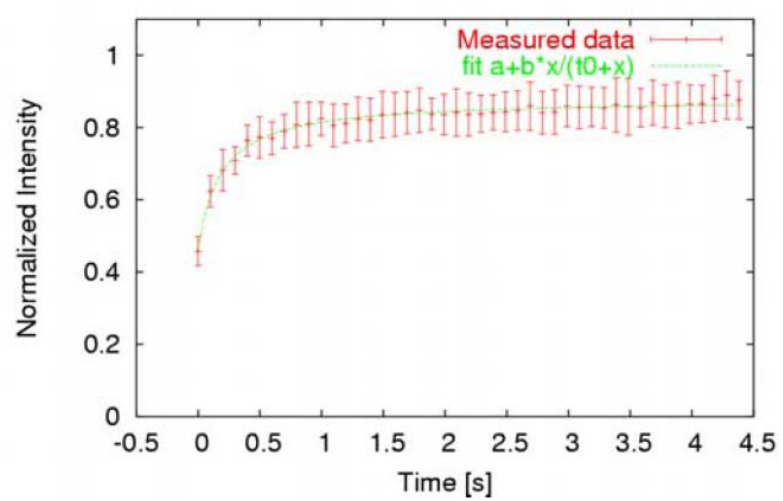

Figure 14. Diffusion of PcGFP in nuclei of preblastoderm embryos. A square shaped region $(1.5 \times 1.5 \mu \mathrm{m})$ in the center of a preblastoderm nucleus (A) of a PcGFP embryo was photobleached and the fluorescence recovery was measured over time. (B) Data points for 40 FRAP curves from similar nuclei as in (A) were averaged and fitted to a hyperbolic function (Equation 2).

Table 4. Diffusion coefficients for PhGFP and PcGFP

\begin{tabular}{l|c|l} 
& $\begin{array}{c}\text { Preblastoderm } \\
\text { embryos }\end{array}$ & $\begin{array}{l}\text { Salivary gland } \\
\text { nuclei }\end{array}$ \\
\hline PCGFP & $\mathrm{D}=0.74 \mu \mathrm{m}^{2} / \mathrm{s}$ & $\mathrm{D}=0.41 \mu \mathrm{m}^{2} / \mathrm{s}$ \\
\hline PHGFP & & $\mathrm{D}=0.22 \mu \mathrm{m}^{2} / \mathrm{s}$
\end{tabular}

From FRAP experiments in both early embryos (Fig. 14B) and salivary gland nucleoplasm (not shown), I obtained similar diffusion coefficients for PcGFP of $0.74 \mu^{2}$ $\mathrm{s}^{-1}$ and $0.41 \mu \mathrm{m}^{2} \mathrm{~s}^{-1}$, respectively (Table 4). The amount of PhGFP induced in early preblastoderm embryos was insufficient to obtain reproducible FRAP measurements. Thus, the free diffusion coefficient was derived exclusively from salivary gland nuclei. The value, $0.22 \mu \mathrm{m}^{2} \mathrm{~s}^{-1}$, is only two times slower than that of PcGFP (Table 4) as would be expected for a protein three times larger in size than Pc. The values for both proteins are slower than expected for free, monomeric, diffusing proteins (Verkman, 2002), indicating that the proteins may interact non-specifically in the nucleus with histones or other 
chromatin-bound proteins although no specific binding to PREs occurs at the early embryonic stage.

\section{III.2.2 Simulation of diffusion processes with various boundary conditions}

Diffusion of molecules in two dimensions has been described previously (Axelrod et al., 1976). In a FRAP experiment on fluorescent molecules localized in a 2-dimensional cellular membrane, un-bounded two-dimensional diffusion coefficients can be obtained from the experimental data using the following expression:

$$
D=\frac{w^{2}}{4 \cdot t_{0}} \cdot \gamma_{D}
$$

where

$$
\begin{array}{ll}
w & \text { bleach box radius } \\
t_{0} & \text { half-maximum recovery time } \\
\gamma_{D} & \text { correction factor for the shape of the bleach area }
\end{array}
$$

Considering boundary conditions, i.e. the finite size of the cytoplasm or nucleoplasm, determination of the diffusion coefficient is more demanding. In addition, presence of the third dimension adds another level of complexity. This simulation was performed in two dimensions to determine the limit where the boundary situation affects the diffusion coefficient calculation from experimental data. Given the $D=0.5 \mu \mathrm{m}^{2} / \mathrm{s}$, 256×256 image size, 8 pixels bleach spot radius or box half-width (Fig. 15A and B) respectively, I simulated diffusion for various “nuclear” sizes (diameters: 16, 24, 32 pixels etc. see Fig. 15B) and extracted the $t_{1 / 2}$ value that was used to calculate $\gamma_{\mathrm{D}}$ (Fig. 15C). The first part of the curve shows that for a low ratio of nuclear size to bleach box size, it would be inappropriate to use the equation 10 to calculate the diffusion coefficient. For ratios $>10$ the gamma curve for the round bleach box approaches the value 0.88 , as predicted by Axelrod's theory. This finding at the same time validates the grid based simulations. In addition I determined $\gamma_{\mathrm{D}}$ values for FRAP experiments where a square bleach box is used 
such as in Fig. 15C (red curve in Fig. 15 C). The ratio of nucleus to bleach box size in the case of the preblastoderm nuclei was 5.5 therefore I needed to use the $\gamma_{\mathrm{D}}$ corresponding to this value to calculate the diffusion coefficient $\left(\gamma_{D}=0.97\right)$. In salivary gland nuclei the ratio was between 6 and $7\left(\gamma_{D}=1.03\right)$.

To determine the half maximum recovery time from simulated or experimental FRAP curves were fitted with various functions. The first observation was that a simple exponential does not fit diffusion recovery curves in cells well. The equations that did fit such curves reasonably well were the stretched exponential (11) and the hyperbolic function (12). I used the hyperbolic function to fit diffusion FRAP curves because it has a well defined slope at time zero.

$$
\begin{gathered}
y=a+b \cdot\left(1-e^{t^{0.5}}\right) \\
y=a+\frac{t}{t+t_{0}}
\end{gathered}
$$

where $t_{0}$ corresponds to the half-maximum recovery time, $a$ is the offset of the curve and $b$ is the amplitude of the recovery curve. 

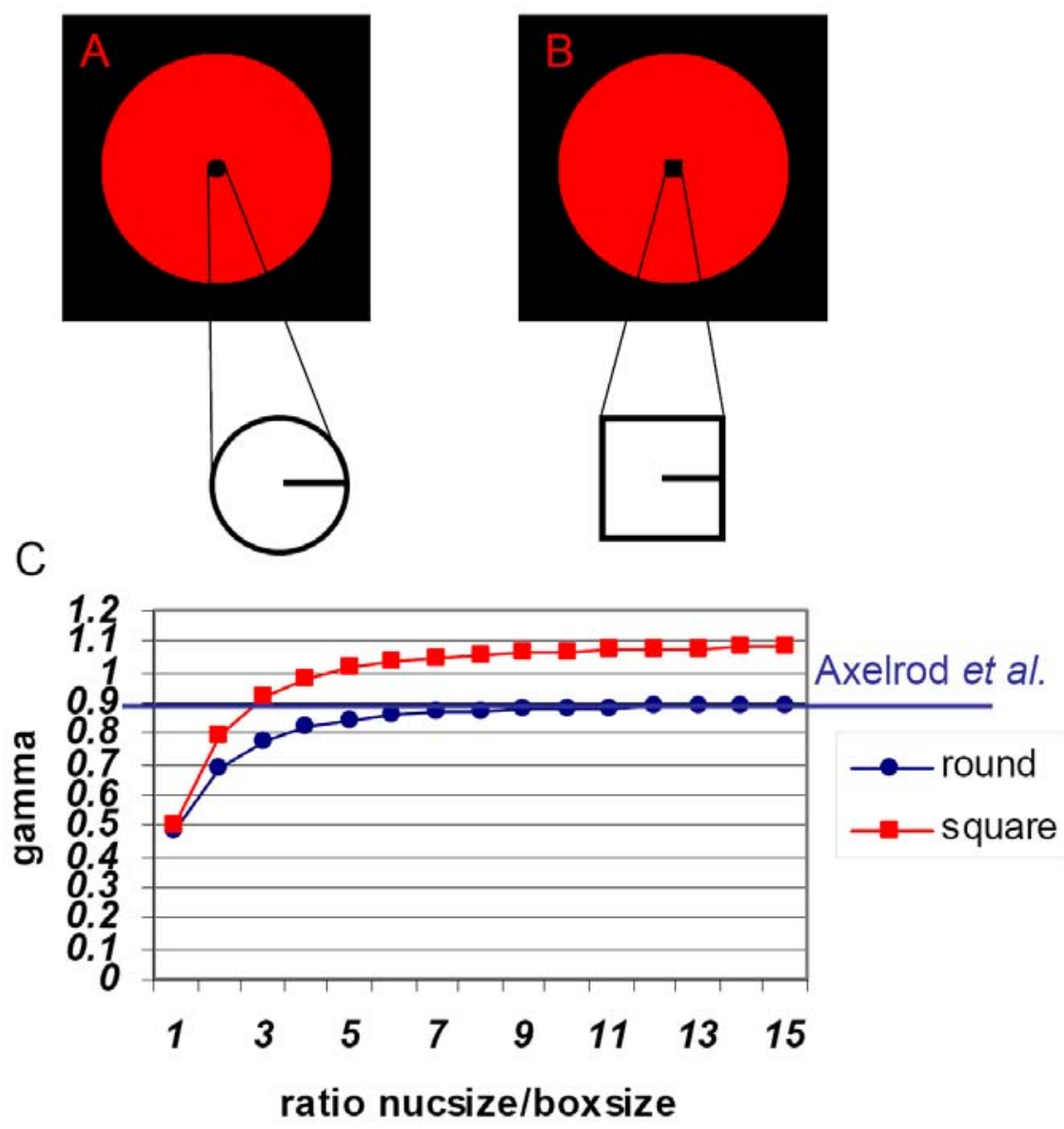

Figure 15. Gamma correction for boundary conditions and box shape. Simulated FRAP in nuclei with round (A) and square bleach box (B) with a fixed diffusion coefficient of $0.5 \mu \mathrm{m}^{2} / \mathrm{s}$. (C) Increasing nuclear/bleach box size ratios have been simulated and $\gamma_{D}$ was calculated using equation (1) for round (blue) and square (red) bleach boxes. Note the blue curve at high nuclear/bleach box ratios limits at the value predicted theoretically by Axelrod et al.,1976.

\section{III.3 Photobleaching in living Drosophila embryos and tissues: possibilities and limitations}

All photobleaching experiments in the literature investigating the dynamic behavior of factors involved in processes such as chromatin remodeling, transcriptional activation, pre-mRNA splicing, rRNA processing and DNA repair were performed in mammalian cell cultures (Dundr et al., 2004; Dundr et al., 2002; Dundr et al., 2000; Kimura, 2005; 
Schmiedeberg et al., 2004; Snaar et al., 2000; Stavreva et al., 2004). This thesis and publications resulting therefrom constitute the first FRAP experiment in Drosophila embryos or tissues. The major difference between mammalian cells and Drosophila cells is the size of the nucleus. The diameter of the nucleus in most mammalian cell is $>10 \mu \mathrm{m}$ whereas somatic post-blastoderm Drosophila nuclei measure a diameter of $4 \mu \mathrm{m}$. As it was demonstrated in chapter III.2.2, to make an accurate measurement of the diffusion coefficient the size ratio nucleus/bleach box should be $>10$ in order to apply equation (10). For the diffusion, the smaller the bleach box the faster the recovery is, thus limiting the measurement of fast diffusion in very small volumes of fluorescence. The simulation of diffusion helped to understand boundary conditions for proper FRAP measurements.

\section{III.3.1 Classical photobleaching techniques (FRAP, iFRAP, FLIP) are not applicable to analyze Drosophila PcG complexes in embryos and larval tissues}

To determine the dissociation and association rate constants of PcG proteins in stages where PcG loci are formed (Fig. 11), I performed a number of photobleaching measurements. In chapter III.1.2 I described the extensive movement of the nucleus and the nuclear content. The basis of FRAP is the photodestruction of the fluorescent molecules in the region of interest, in this case the PcG locus. General nuclear and nuclear content movement will distort the measurement of fluorescence recovery by displacing the region of interest. Therefore a confocal FRAP cannot be performed.

Fig. 16A represents a FRAP experiment performed in wing imaginal discs expressing PhGFP. Most of the initial FRAP data where less movement occurred were recorded when nuclei showed less movement, i.e. after $\sim 2$ hours after mounting. Before this time, the success of performing FRAP was dependent on the unpredicted movement of the locus analyzed. Very often, after the recovery of the fluorescence was seen, slight moving of the knobs controlling the XYZ stage was necessary in order to "keep" the PcG locus in focus. Due to these reasons, FRAP turned out to be a very unreliable measurement in the embryos and imaginal discs to accurately determine the fluorescence intensity of the PcG locus in time. 


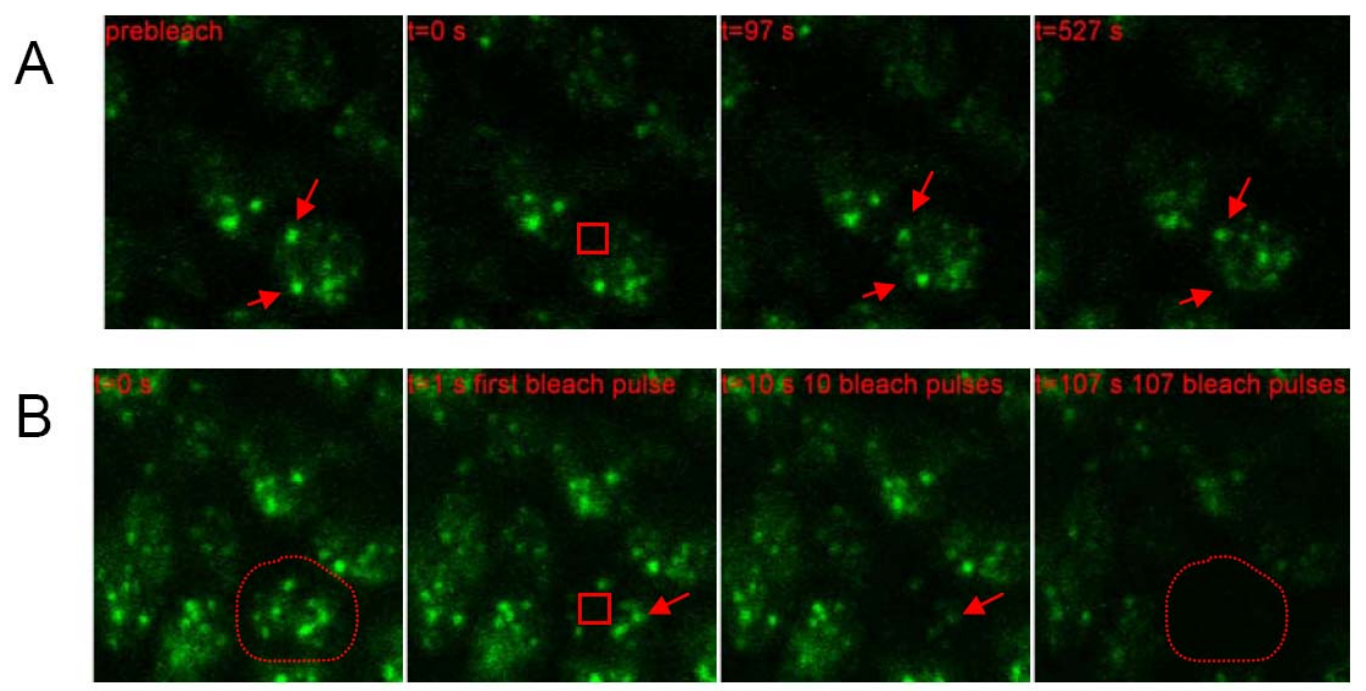

Figure 16. Classical photobleaching techniques in Drosophila embryos and tissues. (A) FRAP in wing imaginal discs. All images are single confocal sections. The prebleach and three different postbleach timespoints are shown. The two arrows point to the two PcG loci and their delocalization. Note that after 500 seconds the unbleached locus is not visible anymore due to nuclear content restructuring. (B) FLIP in wing imaginal discs. High laser intensity pulses have been applied in the red box and dissociation of fluorescence monitored in the rest of the nucleus. Note the fluorescence depletion (also in other nuclei) due to secondary bleach effect.

A three dimensional tracking of the PcG locus intensity would be possible but the difficulty is that the postbleach delocalization of the locus is next to impossible to follow since the locus is not fluorescent anymore. Only when the fluorescence has recovered at some extent, tracking would be possible but under these conditions a manual control of the locus positioning in the tracking program would have been necessary. Another photobleaching method is FLIP (see he methods section for explanations) and an example of an experiment is shown in Fig. 16B. By alternating bleaching of a small region in the nucleus and monitoring the confocal section, dissociation of fluorescence in the unbleached region can be measured.

As it can be seen most of the fluorescence in the nucleus and as well as some fluorescence from other surrounding nuclei has been depleted after 100 seconds. This is termed secondary bleaching (Fig. 17). 


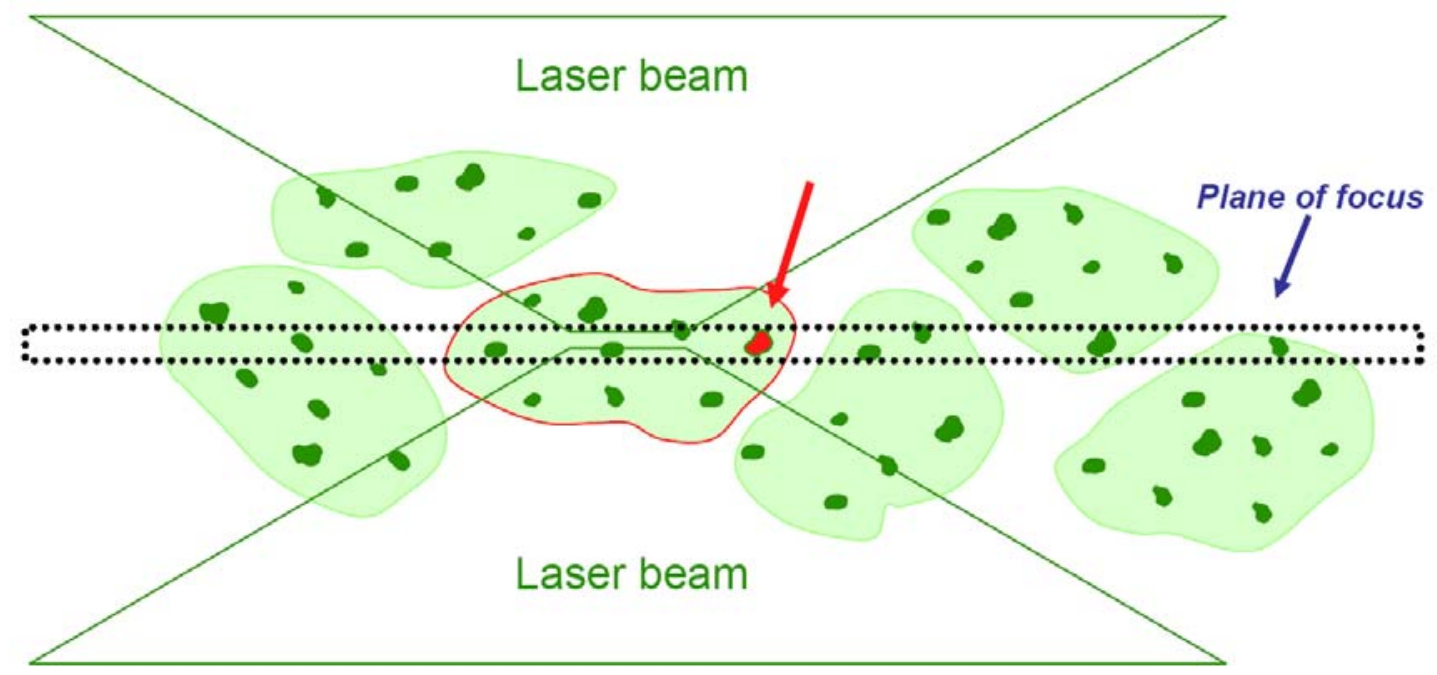

Figure 17. Secondary bleaching. An XZ schematic representation of the field of focus in Fig. 16 is shown here. The nucleus and the PcG locus of interest are depicted in red. The confocal section is delimited by the segmented black line. The multiple high illumination angle of the laser beam (in FLIP) causes destruction of the fluorophores below and above the focus. Movement of the neighbouring nuclei inside this area leads to bleaching of the nuclear content that will be observed in the confocal section. Secondary bleaching might create bleaching at the PcG locus of interest as well.

It is not depletion due to diffusion of fluorescent molecules into the bleach area but photobleaching due to the high NA - wide illumination angle of the laser beam. Changing the frequency of the bleaching in FLIP (i.e. not after every monitoring scan but after 3, 5 or 10 scans did not solve this problem but rather raised the possibility of overestimating the dissociation time of the fluorescent proteins from the PcG loci). The secondary bleaching is not significant in FRAP since there is only one bleaching event at very high laser intensity.

\section{III.3.2 Simulation of the out-of-focus bleaching}

A theoretical analysis of the effect of secondary bleaching by performing FLIP in Drosophila embryonic and imaginal discs nuclei is diagrammed in Fig. 18. The nucleus in Fig. 18B displayed as an $\mathrm{XZ}$ and the photobleaching in performed in the very center of it (dark spot). 
The photo-induced bleaching when multiple bleaching with a high NA objective is far from the cylindrically symmetric (all slices are equal) approximation. In fact the aperture half angle of slightly less than 70 degrees can make this effect quite drastic.

Figure 18B shows a simulated situation of a uniform concentration of fluorophore in a nucleus right after instantaneous bleaching of a square box (down to 20\%) as a central XZ-slice. The fraction of bleached fluorophores over depth is depicted in Fig. 18A. It can be seen that many more fluorophores are bleached in a slice at a certain distance to the plane of focus than directly in the plane of focus. This is caused by a saturation effect caused by the exponential decay of bleaching fluorophores. No fluorophores can be bleached in a region, where everything is already bleached, whereas bleaching over an extended region with lower intensity, albeit for a longer time in slices above and below remains effective.

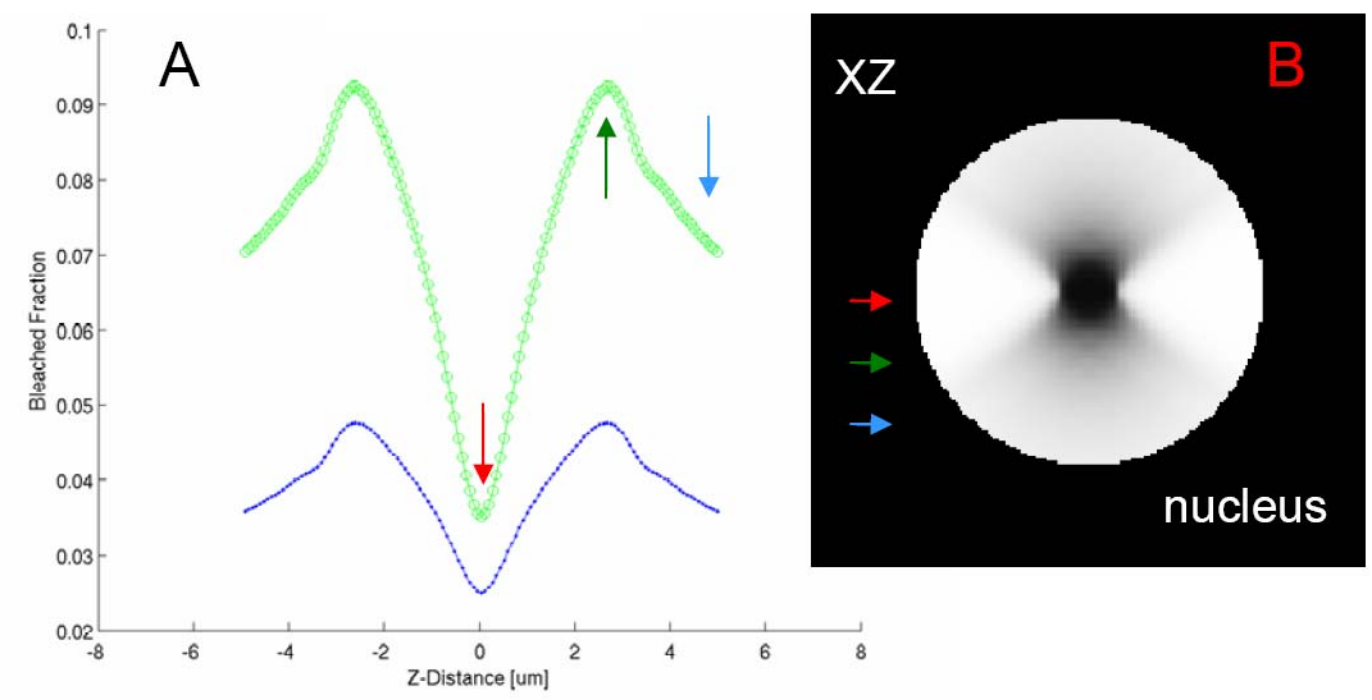

Figure 18. Simulation of the out-of-focus bleaching. (A) Fraction of bleached fluorophores in the simulated nucleus over depth. (B) Simulated photobleaching in the nucleus. Note the higher amount of bleaching at a certain distance from the plane of focus (obtained by Dr. Rainer Heintzmann).

It is not uncommon to model the bleaching point spread function in FRAP as a 3D Gaussian distribution. However, doing so is in clear contradiction to an equal total intensity 
in all planes and would lead to a strong underestimation of the bleaching in out-of-focus planes, which was shown above to be in total even stronger than in the in-focus plane.

In Fig. 18A it can also be seen that the fraction of bleached fluorophores after an initial rise, starts to drop again at distances even further away from the plane of focus. This is due to the influence of the finite size of the nucleus. The area that is affected by the blurred out-of-focus structure of the bleaching region rapidly extends beyond the size of the nucleus, in which case the parts of the beam outside the nucleus will not cause any bleaching; the bleached fraction of fluorophores in a slice decays again. It is an interesting observation that a specific slice at a certain distance from the plane of focus can suffer maximal bleaching. The exact position of this slice of maximal bleaching will depend on the axial position of the plane of focus with respect to the nuclear envelope.

\section{III.3.3 3D-iFRAP: new adapted photobleaching technique to determine dissociation constants of PcG proteins in Drosophila}

Since classical photobleaching techniques (FRAP, FLIP, iFRAP) are not suitable for later developmental stages in embryos and larval tissues, dissociation and residence times of the PcG chromatin bound proteins were analyzed in three dimensions by adapting the iFRAP procedure to a version denoted 3D-iFRAP (see Materials and Methods). A preferred method for determining dissociation rate constants under such conditions is to use inverse FRAP (iFRAP) whereby the entire nucleus except for a small region surrounding the fluorescent complex of interest is bleached and the depletion of fluorescence from this region is monitored over time. The rate of disappearance of the fluorescent locus will be a direct measure of the first order dissociation rate constant of the protein from the complex (Dundr et al., 2002). The fluorescence decay of the unbleached locus and the increase in fluorescence in the bleached nucleus were monitored in the movement corrected data (Fig. 20) and the average intensities of the locus of interest were plotted over time to derive the rate constants (Fig. 21).

Fig. 19 shows how average fluorescence intensity of the PcG locus was calculated in the time points of the 3D-iFRAP. After images were corrected for the movement by tracking the PcG loci over time (Fig. 12), what is left is a series of 3D images where the locus is immobile and only the fluorescence intensity is changing over time (Fig. 20). Instead of averaging the intensity in a two dimensional confocal volume box, prebleach 
and postbleach intensities of the locus were averaged in a weighted volume in 3 confocal slices.

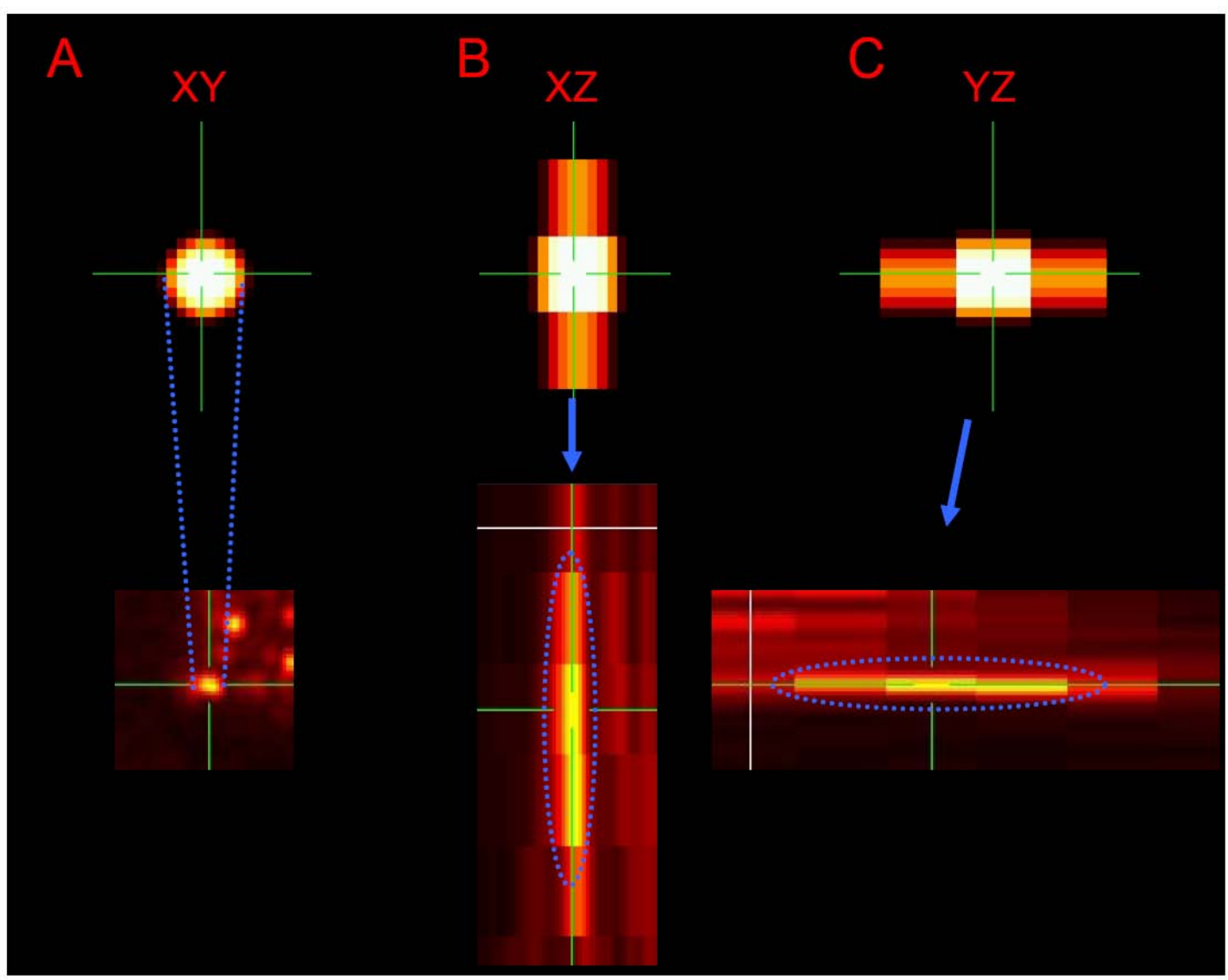

Figure 19. Average intensity calculation in a three dimensional weighted volume. (A) The XY view of the volume of analysis (upper image) and the PcG locus of interest (lower image). The weighted volume consists of a number of pixels comparable to the PcG locus. $\mathrm{B}$ and $\mathrm{C}$ are the $\mathrm{XZ}$ and $\mathrm{YZ}$ views of the volume of analysis (upper image) and the PcG locus of interest (lower image). In the analysis three confocal slices have been considered.

A weighted volume means that the central pixels of the volume "weight" more in that average intensity calculation than the outer pixels. As well, in $\mathrm{Z}$ the weight factor of the middle section of the three $\mathrm{z}$ slices is higher than the upper and lower sections. 
Fig. 20 is and example of the movement corrected data of the 3D-iFRAP experiment. The PcG locus is "immobilized" over time and fluorescence average intensity is calculated at each time-point. The mean intensity was plotted over time for each individual locus and the curves were fitted to a single exponential function characteristic for this process.

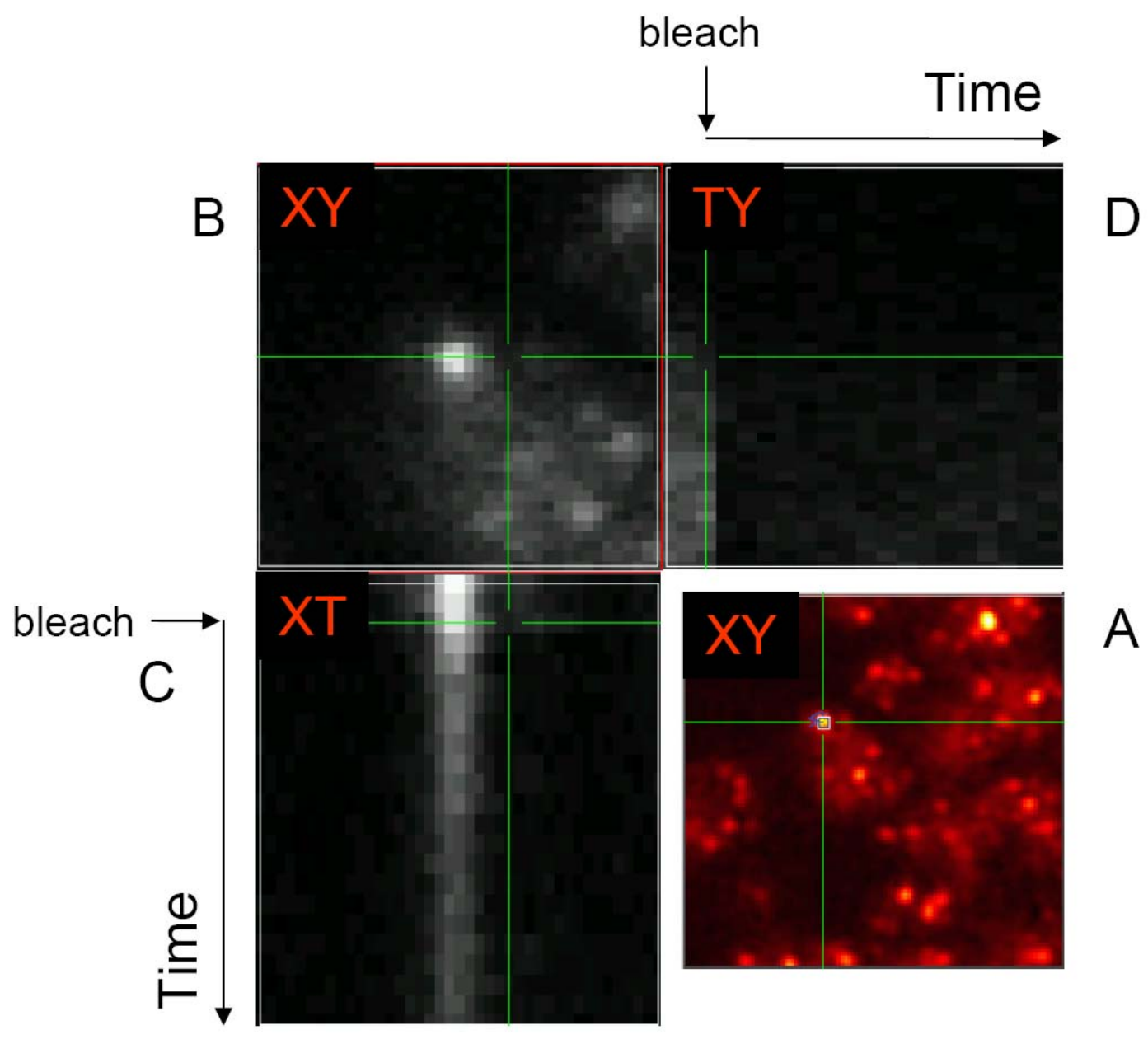

Figure 20. 3D-iFRAP. (A) Overview of part of a wing disc with cross-hair on the fluorescent locus selected for iFRAP. Fluorescence depletion was calculated from a recorded series of Z-stack images over time after bleaching and image registration. (B) Magnified XY image of the nucleus before bleaching. Cross-hair set in the bleach region next to the unbleached locus. The images have been registered to correct for the movement of the chromatin and the nucleus itself and the fluorescent locus is centered in the final analysis image time stack. (C and D) X-time and time-Y views, respectively of the registered images at the slices in the $\mathrm{XY}$ image corresponding to the cross-hair in $\mathrm{B}$. In (C) dissociation of the fluorescent molecules from the spared locus can be seen over time. In 
(D) a bleached region is shown over time. Note the transition from before bleaching to after bleaching indicated by the arrows at a specific time.

Theoretically FRAP and iFRAP yields the same information (see section III. 5), i.e. dissociation of molecules from the binding site. The difference between the two methods is the better signal to noise ratio in the iFRAP curve and shorter bleaching time in the case of FRAP. If there was a possibility to perform the iFRAP bleaching in short time like in the FRAP case the recovery curves would be identical (after "inverting" the iFRAP curve for example) and one would need to account for the diffusion and the rebinding processes that take place in the first time points. By fitting the iFRAP dissociation curve at later time after the bleach (like in 3D-iFRAP) the reassociation process can be safely ignored and the change in fluorescence is due directly to the dissociation constant of the complex.

\section{III.4 Dissociation rate constants of PcG complexes in nuclei of embryos and imaginal discs}

3D-iFRAP was used to analyze the residence times and dissociation rate constants of PcG fusion proteins in embryo and wing imaginal disc nuclei of PcGFP and PhGFP expressing flies. Individual recovery times for PhGFP loci in stage 13 to 16 embryos showed a Gaussian distribution around 20 seconds. Thus, I fit a single exponential decay function to the average data from 30 measurements after normalization to the initial intensities (see Fig. 21B). Such a procedure resulted in a single dissociation rate constant of $\mathrm{k}_{\mathrm{off}}=0.051 \pm 0.004 \mathrm{~s}^{-1}$ in embryos. In wing imaginal disc nuclei of PhGFP expressing flies I found a similar distribution of dissociation rate constants, although, with a slightly positive skewness (Fig. 21A). Averaging 32 individual curves, I fit a single dissociation rate constant of $\mathrm{k}_{\text {off }}=0.032 \pm 0.002 \mathrm{~s}^{-1}$.

Although both PcGFP and PhGFP are localized similarly to 30-40 loci in imaginal disc nuclei, the fraction of free fusion protein differs for the two fusion proteins as seen in Fig. 11.

The iFRAP method requires the complete photo-destruction of all free protein, which results in some unintended collateral bleaching of the fluorescent locus of interest by the high NA objective used in the case of PcGFP nuclei that contain a large amount of free protein, as seen by comparing the pre-bleach and first post bleach intensity measurements in Figs. 21B, C and D. 

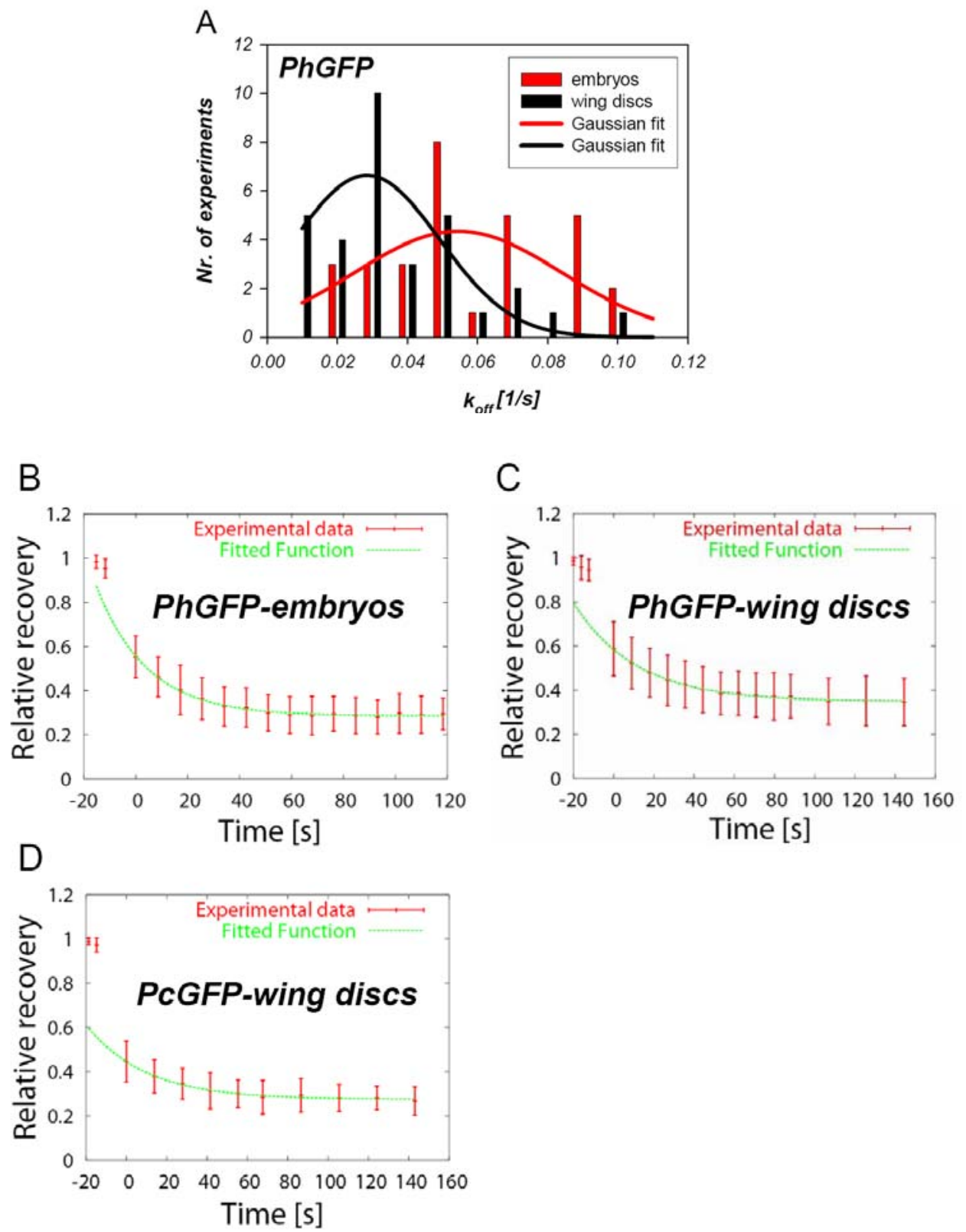

Figure 21. Dissociation rate constants for PhGFP and PcGFP. Individual PcG protein loci in the diploid nuclei of the embryos and wing imaginal discs were subjected to 3D-iFRAP. Fluorescence decay curves were fitted to a single exponential function. (A) Histogram of dissociation rate constants of individual PhGFP loci obtained from 3DiFRAP experiments in embryos and larval wing imaginal discs are shown in red and black, respectively. (B) A single dissociation rate constant could be fitted by averaging all 30 normalized data from PhGFP embryos $\left(\mathrm{k}_{\mathrm{off}}=0.051 \pm 0.004 \mathrm{~s}^{-1}\right)$. (C) Averaging all 32 normalized data from PhGFP wing discs $\left(\mathrm{k}_{\mathrm{off}}=0.032 \pm 0.002 \mathrm{~s}^{-1}\right)$. (D) Average dissociation rate curve for data from 10 determinations of PcGFP loci in imaginal discs $\left(\mathrm{k}_{\mathrm{off}}=0.034 \pm\right.$ $\left.0.003 \mathrm{~s}^{-1}\right)$. 
The reduced fluorescence intensity in the complex decreased the signal to noise ratio for the measurement and reduced the accuracy of the determination of the dissociation rate constant. I compensated for this problem by performing a global fit on all of the dissociation curves for all experiments from the wing discs for the PcGFP. From the dissociation curve depicted in Fig. 21D a dissociation rate constant of $k_{\text {off }}=0.034 \pm 0.003 \mathrm{~s}^{-}$ ${ }^{1}$ was determined, very similar to the dissociation rate constant of PhGFP in larval disc nuclei.

\section{III.5 Model of the binding reactions of proteins to localized chromatin sites}

Fig. 22A and B represent typical confocal images of PcGFP and PhGFP expressing salivary gland nuclei. To investigate the residence time of PcG proteins on their target loci in the genome I performed FRAP experiments on individual bands (results will be described in a later chapter). To obtain quantitative information about the binding of PcG proteins to their binding sites a kinetic model has been developed based on standard principles of chemical kinetics Fig. 23A.
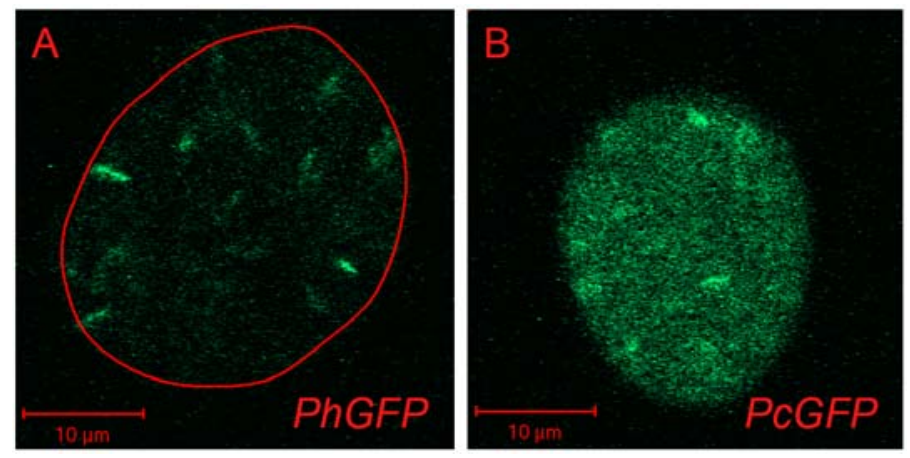

Figure 22. Confocal images of PhGFP and PcGFP expressing salivary gland nuclei. A) PhGFP localizing to discrete binding sites B) PcGFP distribution in salivary gland nuclei

In this model PcG protein complexes bound to their target sites are in chemical equilibrium with the nuclear environment. Dissociation of the molecules from the target 
sites is characterized by the first order rate constant $k_{\text {off }}$ that is determined by the concentration of protein-substrate complex ([PS]).

Formation of the protein substrate complex PS is a result of the association rate constant, $k_{\text {on }}$, that is a second-order rate constant depending on the amount of PcG molecules, [P] and concentration of substrate [S]. Formation of visible PcG loci at the PRE denotes a higher $k_{\text {on }}$ than $k_{\text {off }}$, i.e. a low $\mathrm{K}_{\mathrm{D}}\left(\mathrm{k}_{\text {off }} / \mathrm{k}_{\text {on }}\right)$.

Fig. 23B depicts the processes occurring in FRAP (where one of the fluorescent PcG loci is irreversibly bleached) that was used as one of the assumptions in describing the chemical model in a system of partial differential equations (see Methods). In this model both non-fluorescent (endogenous PcG protein) and fluorescent (GFP fused PcG proteins) compete for the spatially and temporally fixed binding sites with the spatial concentration $C_{s}$. During photobleaching, all fluorescent molecules are irreversibly transformed into nonfluorescent molecules and the FRAP does not change the equilibrium. What will be observed later, after photobleaching, is the binding of the fluorescent molecules from the diffusive pool present in the nucleoplasm. Due to the equilibrium condition this is only possible when the non-fluorescent molecules are dissociating from the target site and leave the binding site free for the diffusing fluorescent molecules (the only ones detected in the process). This means that in the FRAP recovery curve dissociation of the non-fluorescent molecules is directly measured.
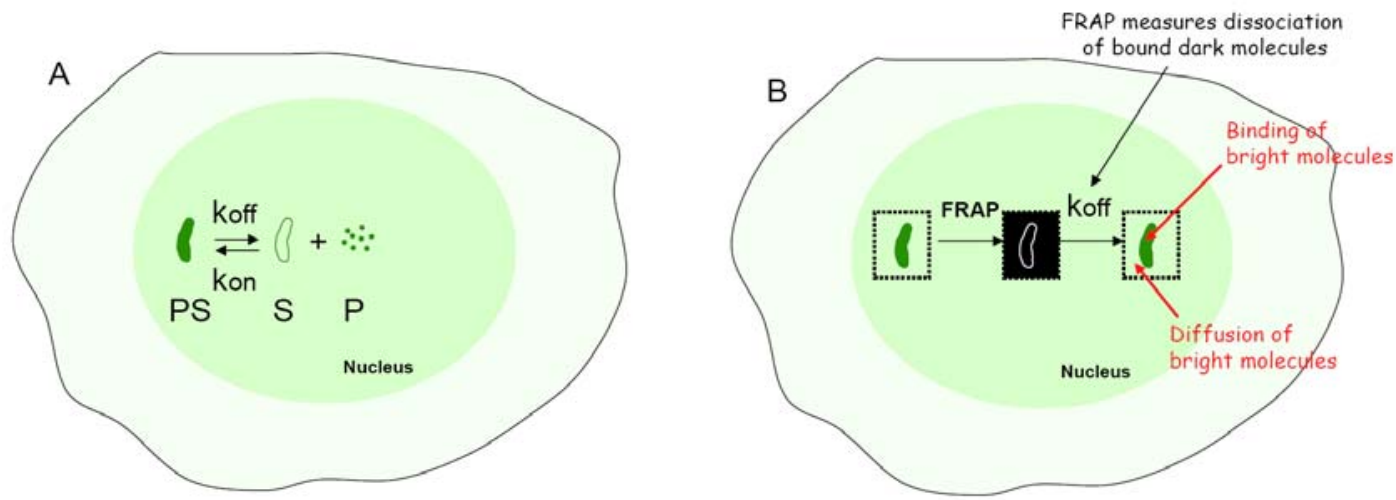

Figure 23. Kinetic modeling of PcG binding to PREs. A) Kinetic model of PcG kinetics. $k_{\text {off }}$, dissociation rate constant of the PcG protein $\mathbf{P}$ from the PRE substrate $\mathbf{S} ; k_{\text {on }}$, association rate constant of the PcG protein $\mathbf{P}$ to the substrate S. B) FRAP transforms all fluorescent molecules in the bleach box into non-fluorescent ones; $k_{\text {off }}$ is the output of FRAP 
The association rate constant of the fluorescent molecules can be determined when the total number of binding sites and the amount of total proteins in the nucleus is known. When the local concentration of binding sites and the equilibrium fraction of occupied binding sites are known for an individual locus, its association ( $2^{\text {nd }}$ order) rate constant can be determined. An estimate of the amount of bound protein can be obtained from the nonperturbed confocal image representing the equilibrium situation (see also the Discussion section).

\section{III.6 Simulation of binding reactions to localized chromatin sites}

In order to gain a better understanding of the binding kinetics of the Polycomb group (PcG) proteins to their target sites in the genome, I simulated the biological situation in the salivary gland nuclei using a grid-based simulation approach (see Methods, chapter II.3.2.3.5). The nuclear distribution of PhGFP and PcGFP is shown in Fig. 22. Due to the polytene nature of these nuclei a band represents a 1000 fold amplification of a particular PcG complex that binds to chromatin at one or a few sites. The value for the diffusion constant of PcG proteins was taken as $0.5 \mu^{2} \mathrm{~s}^{-1}$ from FRAP experiments in embryonic and salivary gland nuclei.

In addition, based on the acquired confocal image I was able to calculate the bound to free ratio of the protein, $B_{\text {ratio }}$ (Fig. 24), of a particular band in a square FRAP box. The ratio between the size of the nucleus and the bleach box could also be determined also from the confocal images. These values were used as starting input values for the simulated FRAP experiments. 


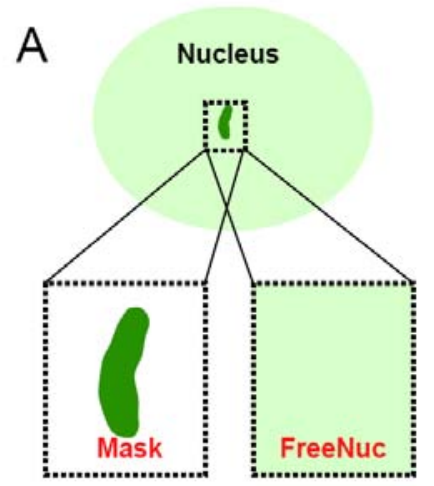

B

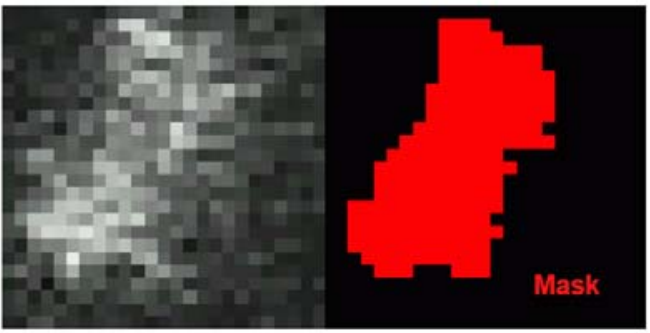

Sum intensity of the bound molecules in mask

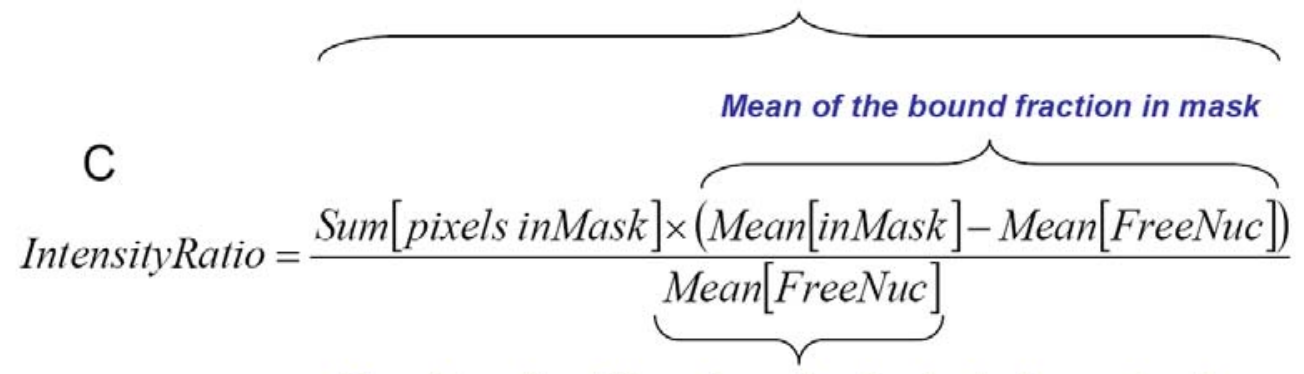

Mean intensity of the unbound molecules in the nucleoplasm

$B_{\text {ratio }}=$ IntensityRatio $\cdot 0.14 \mu \mathrm{m}^{2} / 1 \mathrm{um}^{2}$

Figure 24. $\boldsymbol{B}_{\text {ratio }}$ A) Schematic representation of a confocal image of a salivary gland nucleus with one PcG locus. Mask represents the region where bound molecules are in the segmented confocal image; FreeNuc represents the fluorescence in the nucleoplasm expressed in mean fluorescence intensity. B) Example of segmentation of the PcG locus. The mask that allocates the number of pixels of the bound is shown in red. C) Calculation of the $B_{\text {ratio }} ; 0.14 \mu \mathrm{m}^{2}$ is the pixel size of the confocal image; $1 \mu \mathrm{m}^{2}$ is the pixel size of the simulated image.

\section{III.6.1 Parameters of a simulation experiment}

When setting up a simulation experiment, a number of parameters can be chosen to best represent the biological situation. For example, when simulating free diffusion in preblastoderm embryos the boundary conditions were reduced to a nuclear dimension of $8 \mu \mathrm{m}$ in diameter whereas a FRAP simulation in the salivary gland nuclei was done for a 
nucleus of $25 \mu \mathrm{m}$ in diameter. The importance of the ratio nuclear size/ FRAP bleach box size and the shape of the bleach box can be seen in Fig. 15. The parameters that the user can introduce in a FRAP simulation are shown in Table 5.

The simulation is two dimensional but it covers also a three dimensional situation in which all planes contain identical concentrations of all involved substances and boundary conditions ('cylindrical' symmetry). In simulating FRAP experiments I have used the input parameters as in Table 5 but the program can simulate in essence any initial situation of binding sites, bound proteins, spatially varying amount of bleaching, and even spatially varying diffusion constant.

Table 5. Parameters for a simulated FRAP

\begin{tabular}{|c|c|c|c|}
\hline Parameters & $\begin{array}{l}\text { Simulated } \\
\text { FRAP }\end{array}$ & $\begin{array}{l}\text { Experimental } \\
\text { FRAP }\end{array}$ & Notes \\
\hline $\begin{array}{l}\text { Nucleus radius } \\
\text { (pixels) }\end{array}$ & 80 & $50-90$ & \\
\hline Spot size (pixels) & 2 & variable & $\begin{array}{l}\text { Since the } B_{\text {ratio }} \text { is the important } \\
\text { factor the size of the locus can be } \\
\text { fixed for a particular value }\end{array}$ \\
\hline $\begin{array}{l}\text { FRAP box size } \\
\text { (pixels) }\end{array}$ & 8 & 8 & \\
\hline $\begin{array}{l}\text { Distance between } 2 \\
\text { Spots (pixels) }\end{array}$ & 30 & variable & $\begin{array}{l}\text { Only two spots were used in the } \\
\text { simulation for simplification }\end{array}$ \\
\hline Spot I intensity & $\begin{array}{l}0.8 \\
\text { (from } 0.4- \\
20)\end{array}$ & variable & $\begin{array}{l}\text { The program would return the } B_{\text {ratio }} \\
\text { for a certain intensity (e.g. } B_{\text {ratio }}(\text { for } \\
0.8 \text { intensity) }=6.1 \text {, similar to the } \\
\text { biological situation) }\end{array}$ \\
\hline Spot II intensity & 100 & variable & $\begin{array}{l}\text { The second spot would account for } \\
\text { the rest of the bound proteins in the } \\
\text { nucleus }\end{array}$ \\
\hline$k_{\text {off }}\left(s^{-1}\right)$ & $0.01-0.05$ & $\begin{array}{c}\text { To be } \\
\text { determined }\end{array}$ & $\begin{array}{l}\text { The range of measured } k_{\text {off }} \text { in } \\
\text { diploid nuclei }\end{array}$ \\
\hline Nr. of iterations & $300-2000$ & - & $\begin{array}{l}\text { The longer the better (for a correct } \\
\text { estimation of half-maximum } \\
\text { recovery time it has to have passed } \\
\text { this value) }\end{array}$ \\
\hline $\begin{array}{l}\text { Diffusion coefficient } \\
\left(\mu^{2} / \mathrm{s}\right)\end{array}$ & 0.5 & $\sim 0.5$ & $\begin{array}{l}\text { Value derived from previous FRAP } \\
\text { experiments in preblastoderm } \\
\text { embryos and salivary gland nuclei }\end{array}$ \\
\hline Pixel size $\left(\mu m^{2}\right)$ & 0.14 & 0.14 & \\
\hline
\end{tabular}




\section{III.6.2 FRAP recovery time dependence on the amount of protein bound to the target site}

Using the simulation program one can decompose the FRAP recovery process into the recovery of the free component and the bound fraction. Performing simulation with increasing values of the $B_{\text {ratio }}$ as input parameters while fixing the dissociation constant I observed that the recovery time of the bound fraction was increasing as well. This situation was for a fixed diffusion constant to $0.5 \mu \mathrm{m}^{2} / \mathrm{s}$. The size of the spot was kept constant while only the concentration of binding sites was increased. The range of $B_{\text {ratio }}$ values analyzed was similar to the range determined in the experimental data that is from 5 to 60 .
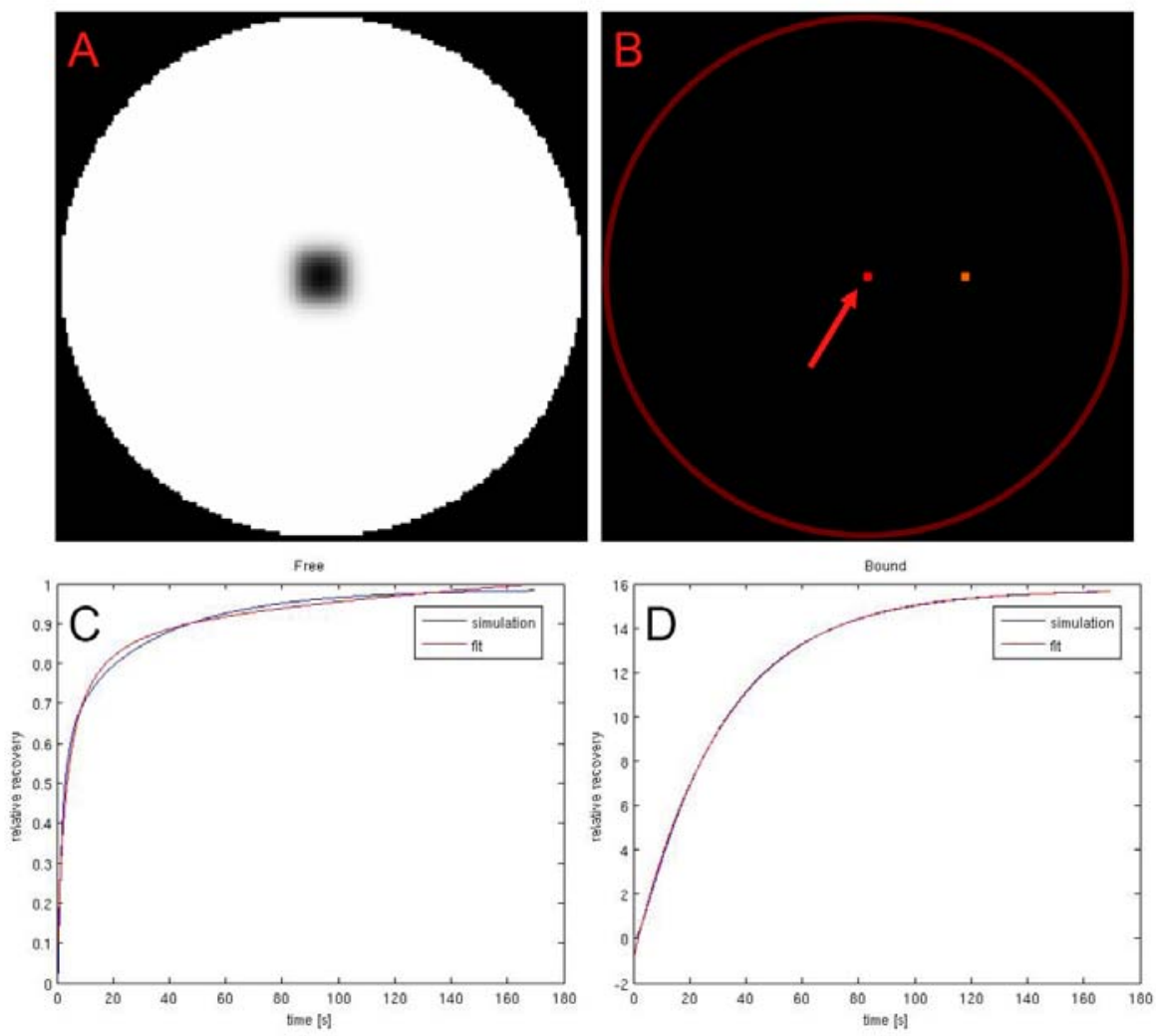

Figure 25. Simulation of the binding kinetics of proteins targeted to localized sites in the nucleus. A and B are segmentations of the same nucleus. A) Represents a snapshot shortly after the bleaching for the free diffusing molecules. B) Bound fractions before bleaching for 2 loci with different numbers of binding sites; one of the spots (red arrow) is the locus of interest. C) FRAP curve for the free diffusing molecules. D) FRAP curve for the binding process. 
Diffusion has a large influence on the rate of recovery of the bound fraction even when assumed to be much faster than binding kinetics (binding-depletion effect). This result calls into questions the validity of the assumptions made by (Carrero et al., 2003; Catez et al., 2004; Rabut et al., 2004). For a large range of diffusion coefficients, the diffusion influences the recovery rate. There is an almost linear dependence of recovery time on the pre-bleach intensity (as determined by the amount of binding sites) in a spot. This renders a uniformly distributed pool of intensity an invalid model. Therefore the compartment model adopted by studies like Catez et al, 2004 is not applicable for situations where the nuclear distribution of bound protein is not uniform.

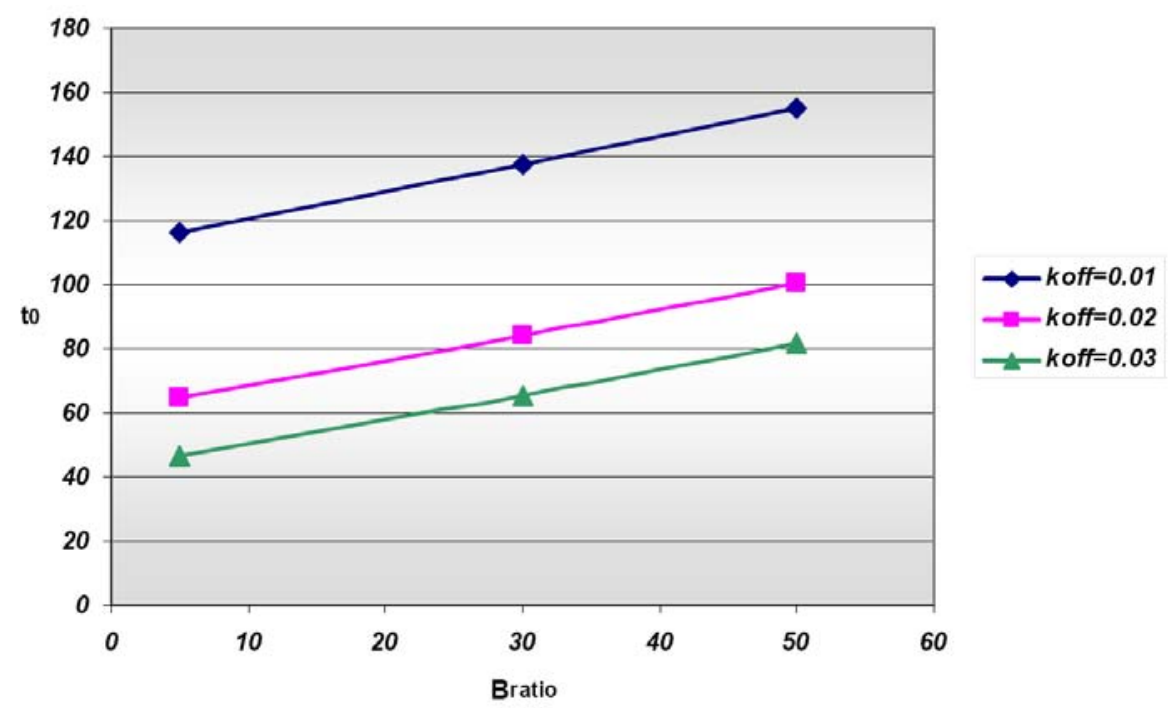

Figure 26 Linear dependence of the recovery time $t_{0}$ on the $B_{\text {ratio }}$. FRAP simulation have been performed by changing alternatively the dissociation rate constant and the $B_{\text {ratio. }}$. Simulations were at a diffusion coefficient of $0.5 \mu \mathrm{m}^{2} / \mathrm{s}$. All other parameters have been kept constant.

The question is, under which conditions diffusion can be assumed to be infinitely fast to describe the exchange kinetics at local binding sites; which range of binding and diffusion parameters allows for such a simplification? Another question is under which conditions (and if at all) diffusion can be approximated as a $1^{\text {st }}$ order kinetic parameter obviating the need for a system of partial differential equations over space? 


\section{III.6.3 The "eating up" effect in FRAP}

The simulation program can separate the two processes: diffusion and binding. When simulating FRAP experiments as shown in Fig. 25, a first observation was that the recovery process of the free component is significantly slowed down when a binding process is active at the same time (Fig. 27A and B). This means that there is a "sink" for molecules in the bleach box where the binding sites are that will attract molecules with high affinity therefore the complete recovery of the free material in the rest of the bleach box will be delayed.

Fig. 27 shows two situations: 1) when the concentration of the binding sites is infinitely small ("no binding”) and 2) when concentration of binding sites is similar to the situation in the salivary gland nuclei ("binding"). The time needed for the free diffusion molecules to replenish the region bleached is much faster in comparison to the situation where the molecules can bind. This is due to a continuous depletion from the surrounding free pool in order to accomplish the binding reaction therefore the recovery of the free component takes longer time than in the absence of binding.

Similarly, the recovery of the bound component is significantly slowed down by the influence of diffusion, which transports material over long distances to the binding sites. If diffusion were infinitely fast in living nuclei, such effects would not occur. 
No binding
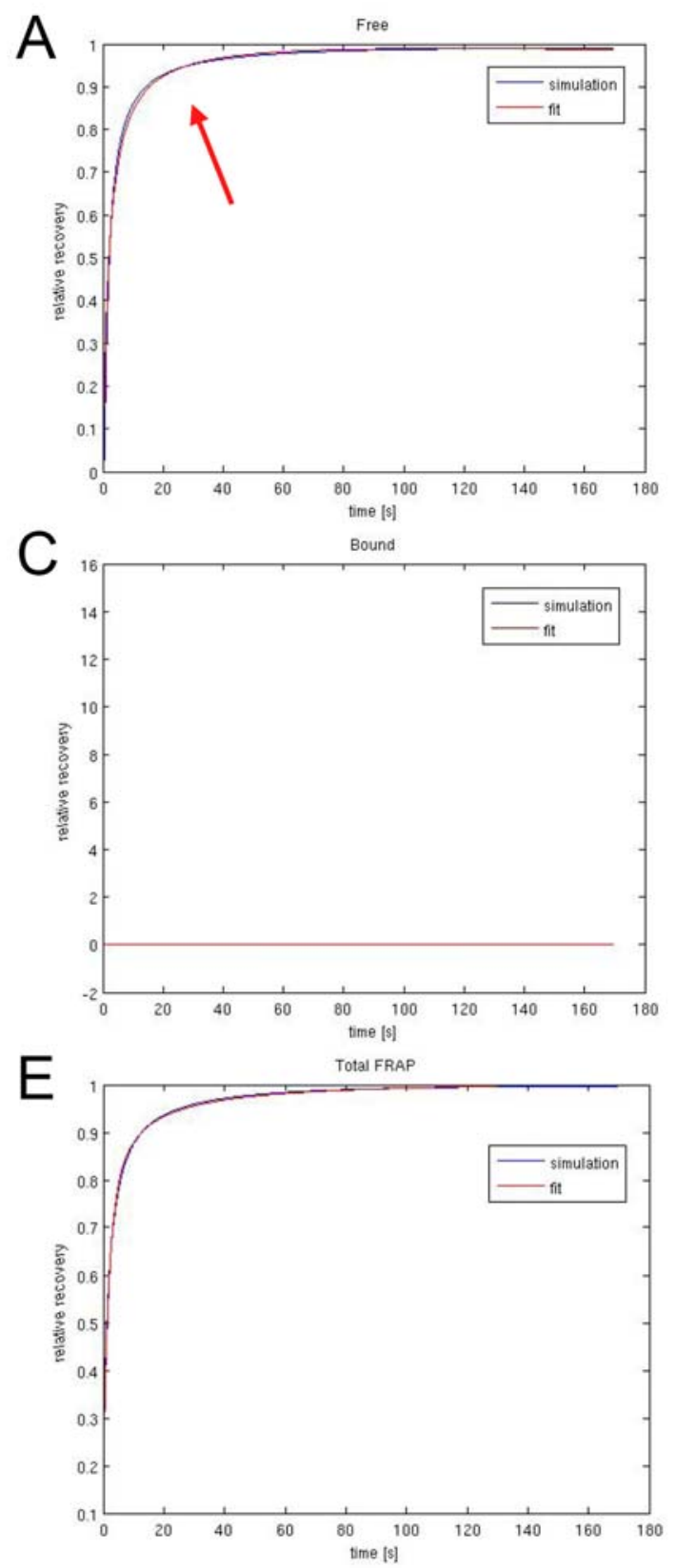

Binding
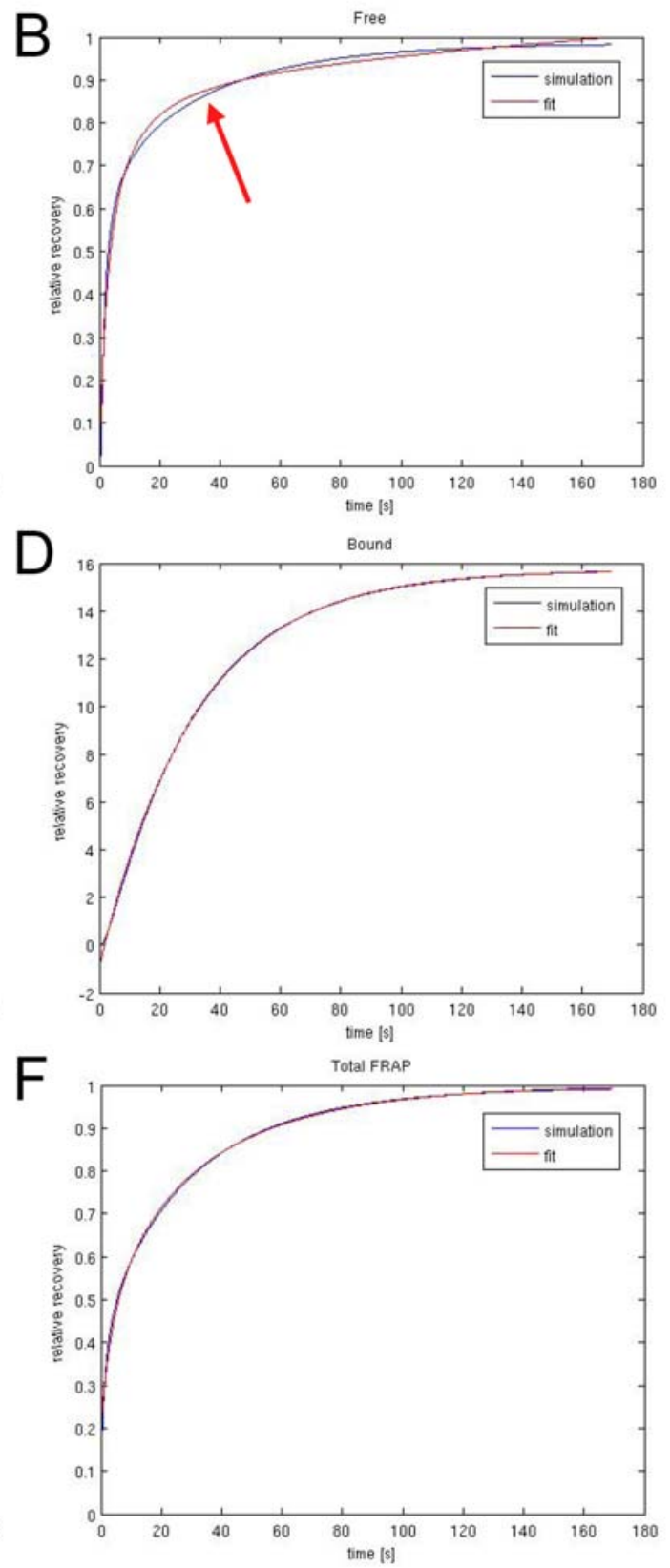

Figure 27. Binding processes slow down the FRAP recovery profile for the free diffusion molecules. FRAP recovery curves for the free diffusing molecules $(A, B)$, the binding process (C.D), and the total FRAP recovery curves, containing the sum of both fractions $(\mathrm{E}, \mathrm{F})$. Two situations are shown: where no binding takes place $(\mathrm{A}, \mathrm{C}, \mathrm{E})$ and where binding is present $(\mathrm{B}, \mathrm{D}, \mathrm{F})$ 


\section{III.7 Distribution, dynamics and dissociation rate constants of PcG proteins on Drosophila polytene chromosomes}

\section{III.7.1 PcG complexes have different residence times on individual bands in salivary gland nuclei}

PcGFP and PhGFP bind to distinct (Fig. 28), overlapping loci in salivary gland nuclei. Classical FRAP experiments (intense photobleaching of the band and monitoring of the fluorescence recovery over time) were conducted on individual bands to determine the equilibrium constants for PcGFP and PhGFP complexes (Fig. 28B and D).

Individual bands showed consistent recovery times after multiple bleachings (Fig. 29B), although, not all bands in the same nucleus exhibited the same recovery times.

The equilibrium association constant of the complex is given by the ratio of a pseudo first-order forward rate constant (that includes the concentration of the free protein) and a first-order dissociation rate constant (see section II. 3.2.2). In the case of PcGFP a large amount of the labelled protein is free, i.e. leading to a lower fraction of bound protein than for PhGFP (bound ratio, $B_{\text {ratio, }}$ see section II. 3.2.2 and Fig. 24).Therefore, the shape of the FRAP recovery curve will be dominated by the fast diffusion constant of the free protein as is seen by comparison of the curves in Fig. $28 \mathrm{C}$ and D. By accounting for the free protein component (Fig. 30) (as described in section II. 3.2.2 and further explained in the Discussion), I computed recovery times ( $t_{0}$ - the time required for the fluorescence intensity to reach $\sim 63 \%$ of the final height of the recovery curve for the bound molecules) for both PhGFP and PcGFP from such curves, Fig. 31A.

The distribution of values was very similar for both proteins with half maximal recovery times ranging from 50 to 350 seconds.

In the case of salivary gland polytene chromosomes the individual bands represent complexes binding to one or a few genes. Thus, I can ask if the range of measured recovery times represents different exchange rates for different genes (PREs) or the same exchange rate influenced by the density of binding sites. 

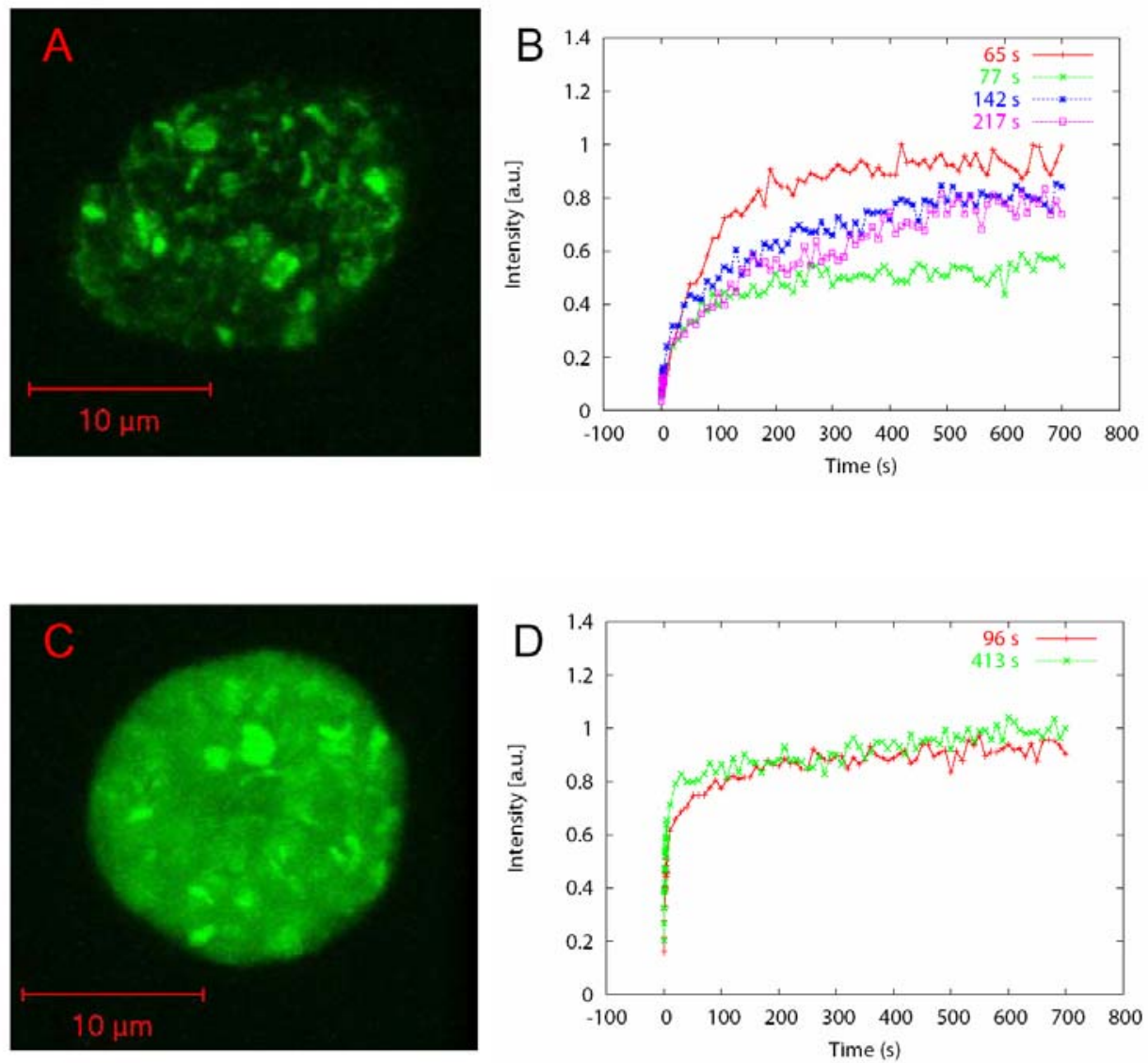

Figure 28. Distribution PhGFP and PcGFP in the larval salivary gland nuclei and FRAP curves for individual bands. Maximum intensity projections of the fluorescence in whole salivary gland nuclei are shown in (A) (PhGFP) and (C) (PcGFP). Note the difference in the amount of the background fluorescence (the unbound protein). Typical FRAP curves from individual PcG loci are shown in (B) (PhGFP) and (D) (PcGFP). The amount of background fluorescence substantially changes the aspect of the recovery curves.

Simulations revealed that for interpreting these FRAP experiments the complex interplay between unbleached free protein, the total amount of bound protein at a locus and the free diffusion constant must be taken into account. I simulated the expected FRAP behaviour for loci with different amounts of bound protein at a locus (thus different intensities) as described in section III.6.2 using a diffusion constant of $0.5 \mu^{2} \mathrm{~s}^{-1}$ of the free protein determined experimentally, and systematically varying the dissociation rate constants around the experimentally determined values. 

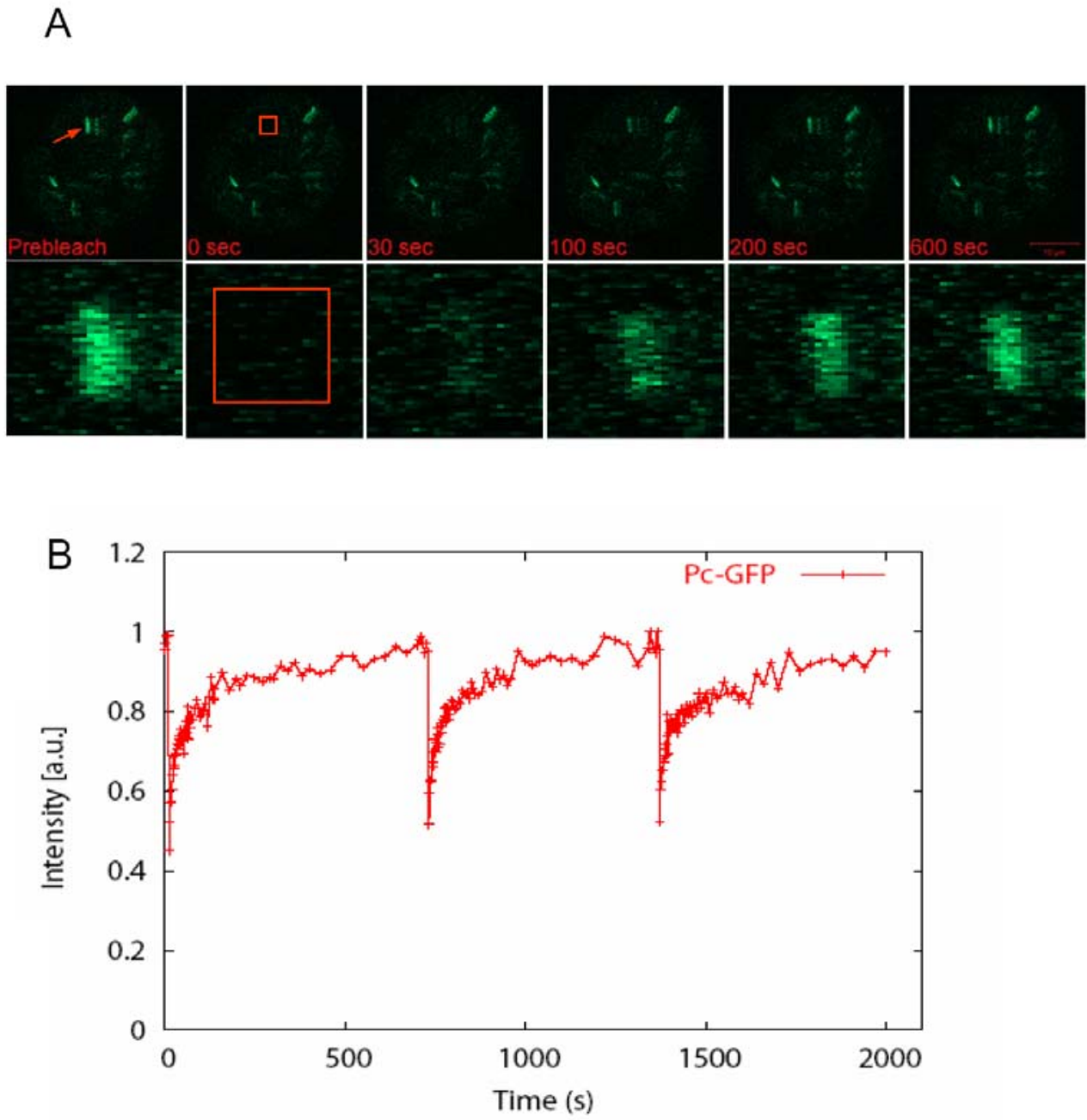

Figure 29. FRAP on the same PcG locus in the salivary gland. Images from selected time points in a typical FRAP experiment (PhGFP in this case) are shown in (A). Upper panels, overview of part of nucleus with bleach box indicated in the second image. Lower panels, magnified region used for analysis. (B) FRAP curves for sequential bleaching of a single PcGFP locus showing reproducible reassociation kinetics.

Fig. 31B demonstrates that with a single dissociation rate constant I would expect the recovery times to vary approximately linearly with the ratio of bound to free protein, $\mathrm{B}_{\text {ratio, }}$ and results in the data shown by the connected points. In Fig. 31B the experimentally measured recovery times are also plotted against the $B_{\text {ratio }}$ for each locus. In the experimental data no strong correlation was found between the recovery time and the intensity of the locus (number of binding sites), indicating that the complexes on different genes have different stabilities, and implying that they differ in composition. 

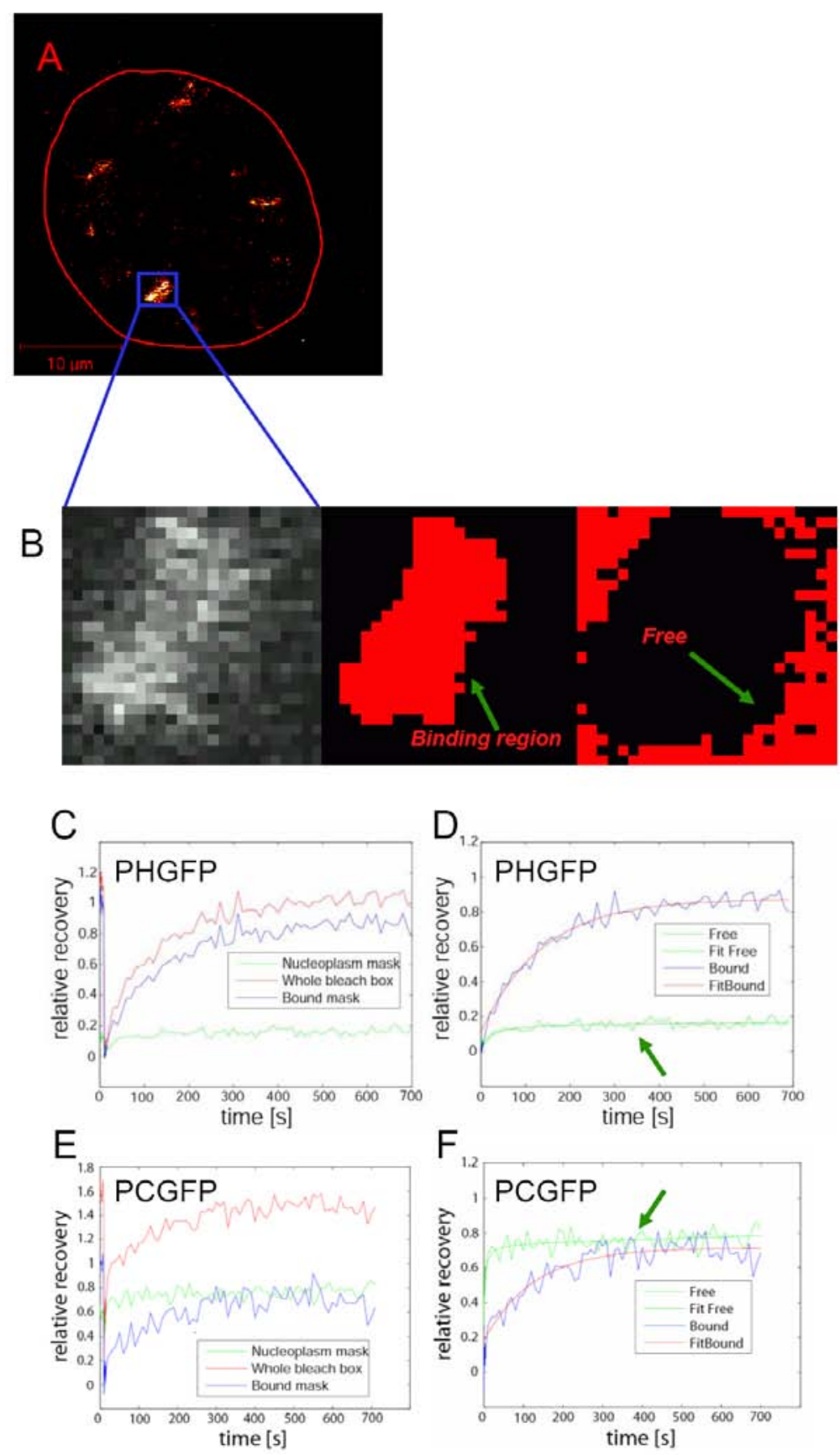

Figure 30. Segmentation of the bound fraction and fitting of the FRAP curves. A confocal image of PhGFP in a salivary gland nucleus is shown in (A). (B): segmentation of the bleach box area into bound and unbound fluorescence regions. (C-PhGFP, EPcGFP): plot of the intensity for all pixels (red curve), the $30 \%$ highest pixels, bound fraction (blue curve), and the $30 \%$ lowest pixels (green curve). (D-PhGFP, F-PcGFP): fit of the bound fraction of the protein to a single exponential (blue curve). Note the difference in the height of the green curve (unbound component) for PhGFP and PcGFP. 


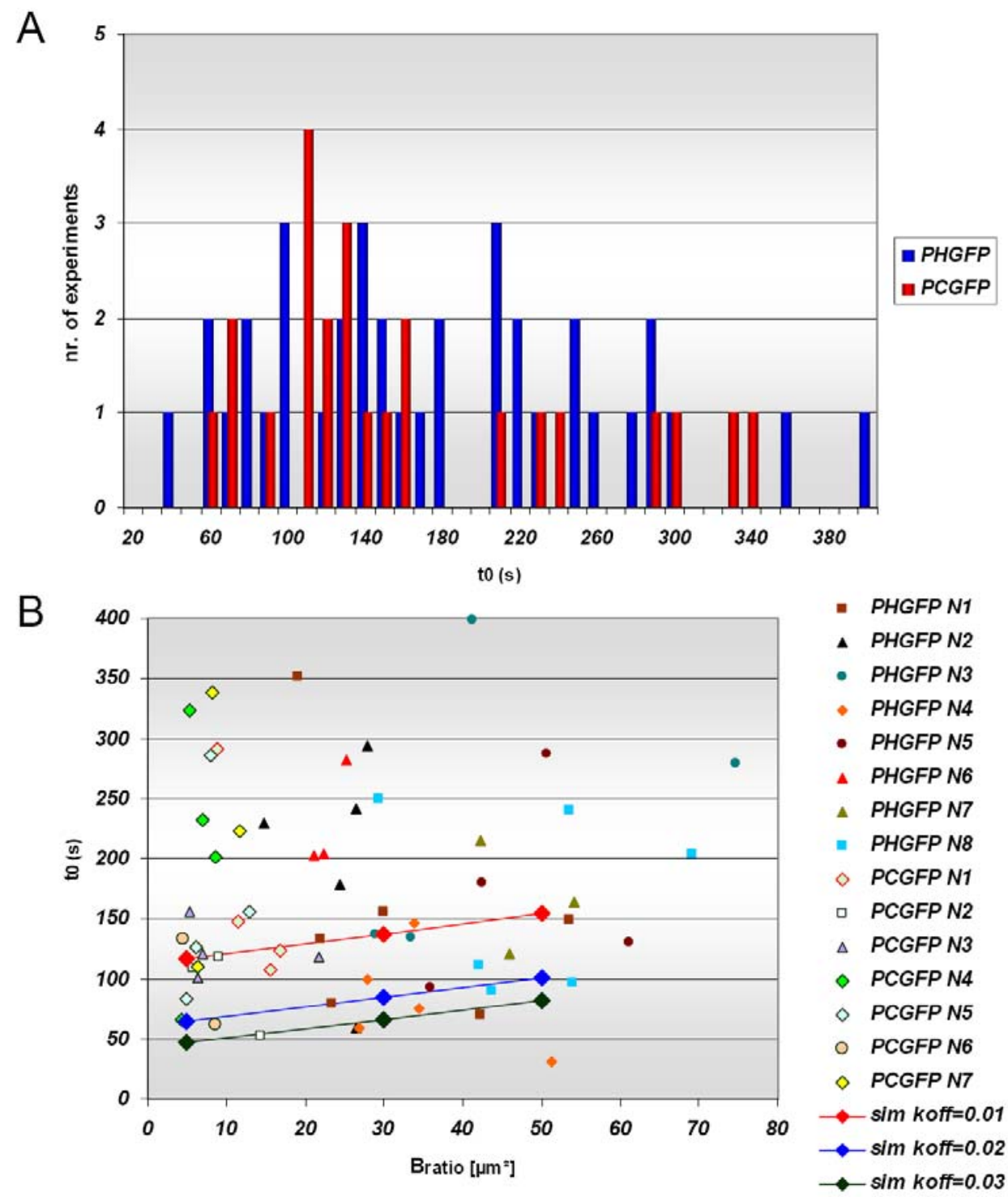

Figure 31. FRAP on bound PcG proteins in the salivary gland nuclei. Individual bands in the salivary gland nuclei were photobleached and redistribution of fluorescence was measured over time. Recovery times $\left(t_{0}\right)$ were plotted as a histogram in (A) (red-PhGFP) and (B) (blue-PcGFP). Redistribution of the fluorescence in both cases occurs in less than 6 minutes. B) Lack of correlation between the concentration of binding sites and recovery time $t_{0}$. Individual nuclei where several loci were analyzed are colour coded (solid coloured symbols for PhGFP and open coloured symbols for PcGFP). Data from simulations show a linear dependence of the $t_{0}$ on the concentration of binding sites, connected points for three different dissociation rate constants. 

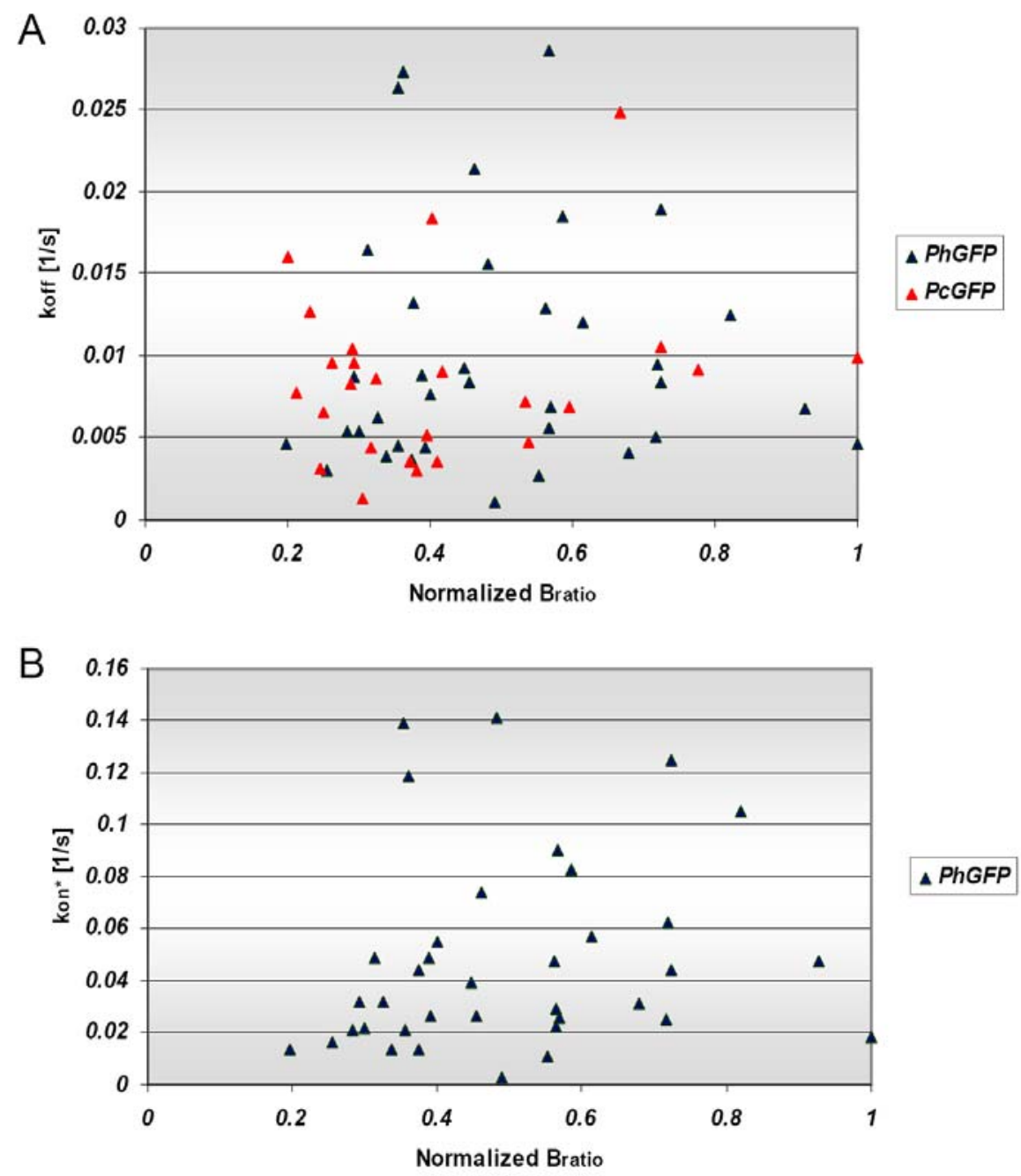

Figure 32. Dissociation rate constants for PhGFP and PcGFP and reassociation rate constants for PhGFP. (A) Dissociation rate constants for PhGFP (blue) and PcGFP (red) plotted against the normalized concentration of binding sites for individual loci. (B) Pseudo-reassociation rate constants for PhGFP (blue). See text for details.

Including the dependence of the dissociation time on the density of binding sites,

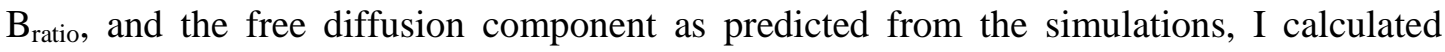
dissociation rate constants for each of the analyzed loci using equation 6 from the section II.3.2.2. These values are independent of the local concentration of sites and the effect of diffusion. Bound protein ratios were normalized to the highest $B_{\text {ratio }}$ value for each protein separately and are plotted in Fig. 32A. 
The dissociation rate constants are similar for both proteins but about $1 / 5$ the values found for the complexes in $2 \mathrm{~N}$ wing disc nuclei. The forward reaction is 2 nd order and the rate is dependent on the concentration of binding sites and on the local concentration of the free protein. The amount of unbound protein in the nucleus is sufficient for the binding process to occur undisturbed for both PhGFP and PcGFP cases. From the fluorescence intensity ratios I calculated that $\sim 10 \%$ of the total PhGFP and $\sim 2 \%$ of the PcGFP are in a bound state in a salivary gland nucleus at equilibrium. Since the absolute number of binding sites (Cs) is unknown, the association rate constant could not be determined independently.

Instead, a pseudo-association rate constant was calculated (which is related to the actual $k_{o n}$ by the equality $k_{o n}{ }^{*}=k_{o n} C_{s}$, equation 7, and Sprague et al. 2004) for each locus and these values for PhGFP are plotted in Fig. 32B. The pseudo-association rate constants are an order of magnitude larger than the dissociation rate constants indicating that dissociation is rate limiting.

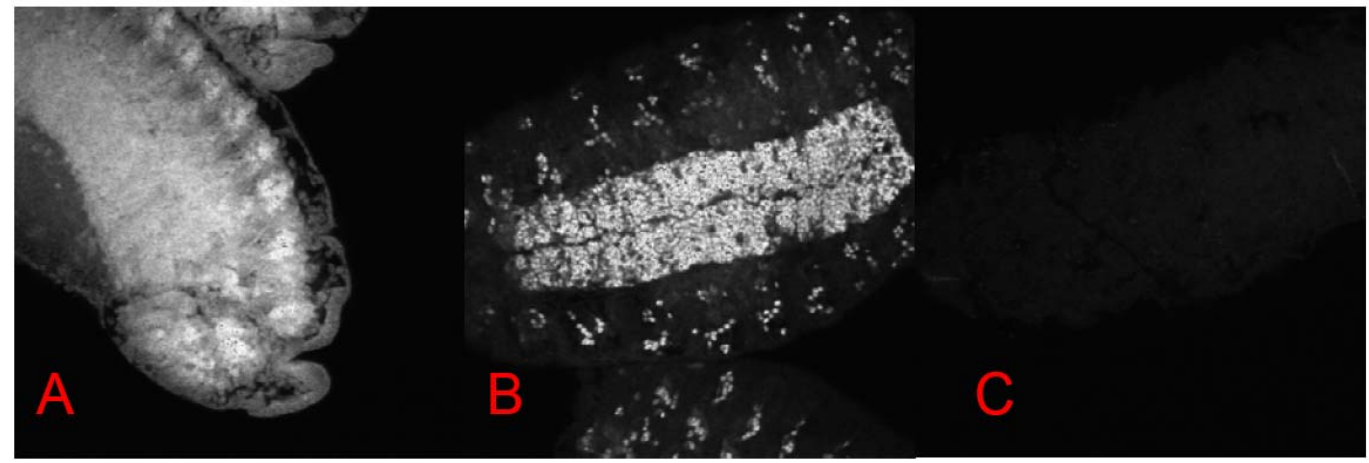

Figure 33. Polyhomeotic protein binding to chromatin requires the presence of the Polycomb protein. (A) Immunostaining of wt larvae for $\mathrm{Ph}$. (B) $\mathrm{Ph}$ protein is restricted to the dividing neuronal cells in heterozygous Pc3 mutants. (C) No Ph protein is detected in homozygous Pc3 larvae (aquired by Peter Buchenau).

\section{III.8 Rescue of the ph null phenotype with phGFP}

The Polyhomeotic protein is strongly required both maternally and zygotically for normal embryonic development. Mutation in both $p h-p$ and $p h-d$ genes is necessary to produce a null mutation. Single-event mutations (in only one of the ph genes) are viable as males and homozygous females; such mutations produce transformations similar to those 
of known dominant gain-of-function mutants in the ANT-C and BX-C, i.e. transformation of wings to halteres, second and third legs to first legs, and anterior abdominal segments to more posterior segments. Two-event mutations are lethal in mid embryogenesis and completely lack ventral and abdominal epidermal derivatives (Dura et al., 1988).

I have used the $p h^{504}$ allele (Dura et al., 1987) to test whether PhGFP is able to rescue the ph null mutation. The mutated allele is kept over the $F M 7 \mathrm{cw}^{\mathrm{a}} \mathrm{B}$ balancer: $\frac{p h^{504} w^{-} F R T 101}{F M 7 c w^{a} B}$, therefore only $p h^{504}$ heterozygous or FM7 homozygous females and $B^{-}$ males survive. $B^{+}$males and females are lethal because they do not have any of the $p h$ genes. The crossing scheme is depicted in detail in the Methods section. I have used the $P\left\{G A L 4 ; w^{+}\right\} B x^{M S 1096}$ driver to drive expression of phGFP in larval imaginal discs for FRAP experiments and thus also used the same driver for the rescue experiment too. Since both the driver and the ph null allele are on the $\mathrm{X}$ chromosome, a recombination step was necessary to bring them on the same chromosome. Luckily the ph genes are located in the distal region of the $\mathrm{X}$ chromosome (2D2-3) and the integration site of the driver was on a region closer to the centromere therefore recombination was possible.

Table 6. ph null phenotype rescue statistics

\begin{tabular}{|c|c|c|c|}
\hline $\begin{array}{c}\text { Nr. of } \\
\text { independent } \\
\text { crosses }\end{array}$ & Resulted genotypes in each cross & $\begin{array}{c}\text { Eye } \\
\text { phenotype }\end{array}$ & Total flies \\
\hline \multirow[t]{4}{*}{5} & ○ $\frac{F M 7 c w^{a} B}{w^{-}} ; \frac{U A S: P c: p h G F P w^{+}}{+}$ & $\boldsymbol{B}^{-}$ & 721 \\
\hline & $\uparrow \frac{p h^{504} w^{-} P\left[M S^{1096} w^{+} G a l 4\right]}{w^{-}} ; \frac{\text { UAS :Pc : phGFP } w^{+}}{+}$ & $\boldsymbol{B}^{+}$ & $\sim 40$ \\
\hline & $\widehat{\jmath} \stackrel{F M 7 c w^{a} B}{\longrightarrow} ; \frac{U A S: P c: p h G F P w^{+}}{+}$ & $\boldsymbol{B}^{-}$ & 260 \\
\hline & $\lesssim \frac{p h^{504} w^{-} P\left[M S^{1096} w^{+} G a l 4\right]}{\longrightarrow} ; \frac{U A S: P c: p h G F P w^{+}}{+}$ & $\boldsymbol{B}^{+}$ & $\begin{array}{c}\sim 10 \\
(\sim 2 / \text { cross })\end{array}$ \\
\hline
\end{tabular}

The recombination outcome was $50 \%$, i.e. half of the single virgin crosses recombined that could be identified in the next cross (see Methods). 
The statistics of the rescue experiment is shown in Table 6 . The last cross between the homozygous phGFP flies and the line containing the GAL4 driver and the $p h^{504}$ null allele has been done in five independent crosses from different recombinant single female generations. $\boldsymbol{B}^{+}$males have survived in each independent cross, i.e. phGFP expression rescued the null lethal phenotype in these flies.

The control cross was performed in the same genotypic background except that instead of the $p h^{504}$ allele a wild type allele was present (see Materials). This cross results in a normal distribution of genotypes in the F0 generation with a high number of $\mathbf{B}^{+}$males but the same number of $\mathbf{B}^{+}$females. The low number of the $\mathbf{B}^{+}$females will be discussed in the following chapter.

\section{III.9 Overexpression of PhGFP induces apoptosis in all developmental stages of Drosophila}

Using the Pc promoter to drive expression of phGFP at endogenous levels was successful only in salivary gland nuclei whereas in embryos and larval diploid tissues, PhGFP was detected only at very low levels. Therefore inducing PhGFP expression in these tissues required the use of specific GAL4 drivers. All GAL4 drivers I used (en$G A L 4$-for embryos, $B x^{M S 1096}$-GAL4-for wing and haltere imaginal discs, ey-GAL4-for eye imaginal discs) resulted in an overexpression phenotype. In summary, when driving expression of PhGFP in embryos using the en-GAL4 driver, all the offspring die as pharate adults. All pupae were 30\% shorter than wt (data not shown). Overexpression of PhGFP in eye discs resulted in very small eyes compared to the wt situation (Fig. 34A and B); the penetrance of this phenotype was 100\%, i.e. all flies overexpressing PhGFP showed lower number of omatidia than wt flies. 

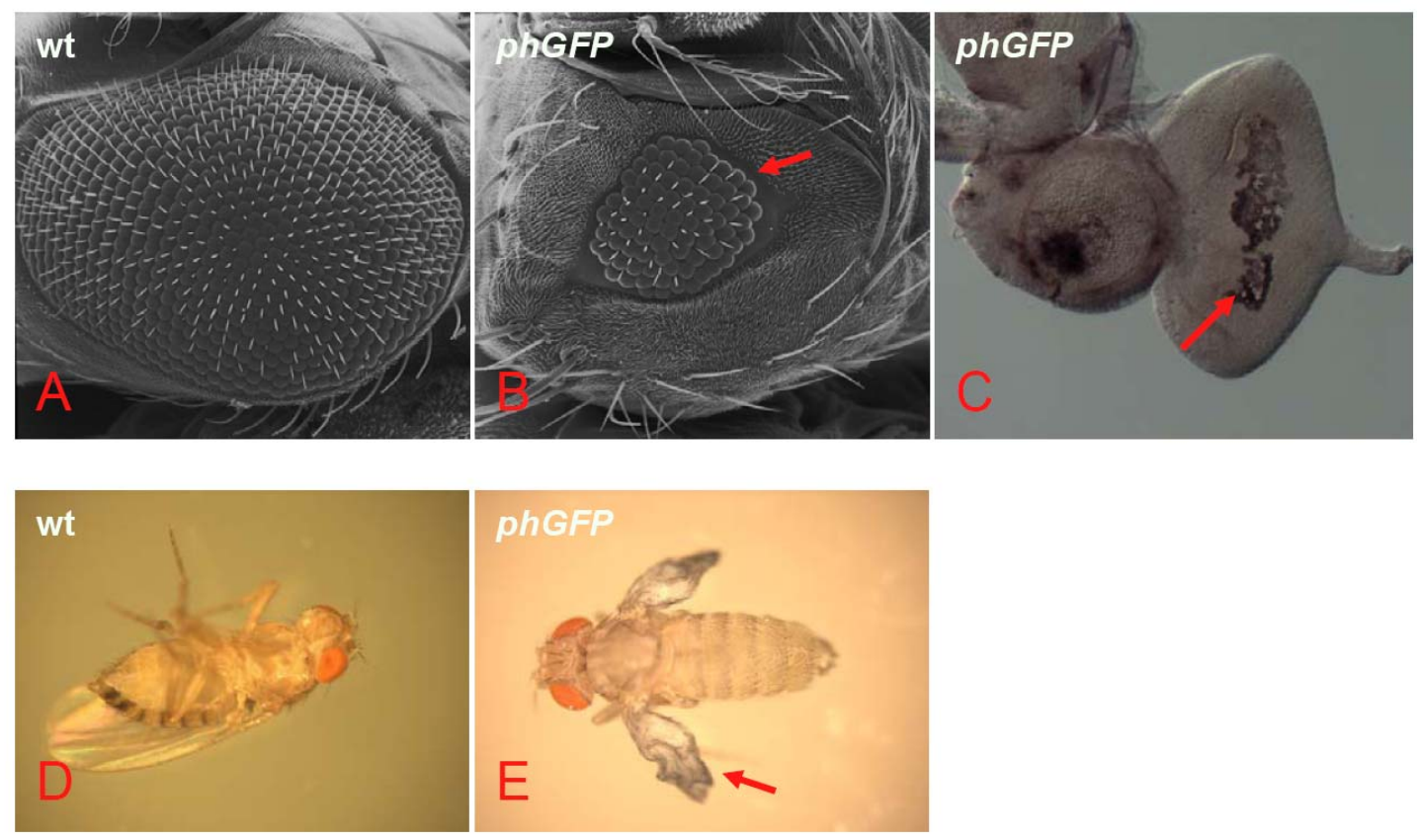

Figure 34. Effects of PhGFP overexpression in the fly eye and wing. Scanning electron micrograph (acquired by Dr. Peter Schwartz) of eyes from wt (A) and PhGFP flies crossed to ey-GAL4 driver fly (B) (Magnification: x 500). C) TUNEL assay to detect apoptotic cells in the eye-anntennal imaginal discs of flies overexpressing PhGFP. Note the apoptosis in the morphogenetic furrow of the eye disc. D) wt fly. E) Necrotic wings in the adult fly overexpressing PhGFP under the $B x^{M S 1096}$-GAL4 driver.

Destruction of marginal omatidia was caused by apoptosis in the morphogenetic furrow (Fig. 34C-red arrow). Overexpression of PhGFP in wing imaginal discs was partially lethal (not all adults survived when crosses were kept @ $22^{\circ} \mathrm{C}$ and this effect was even stronger at 25 and $29^{\circ} \mathrm{C}$ where all adults died). All adults that survived showed morphological changes of the wing blades with various degrees of severeness (from less transformed wings that showed missing veins to very severely transformed wings). Although one would expect a higher degree of apoptosis than in eye discs, it was not significant in this case. Other drivers used in Juerg Mueller's group gave the same result: using vg-GAL4 that drives $\mathrm{Ph}$ expression in wing and haltere discs resulted as well in pharate adults; there were some transformations in the thorax and halteres were partly transformed towards wings. Ubx expression was lost in haltere discs (personal 
communication) therefore it is possible that overexpression of PhGFP silences HOX and PcG target genes important for normal development of the fly.

Various forms of cellular stress lead to activation and stabilization of the p53 tumor suppressor protein and to growth arrest and apoptosis (Lavigne et al., 2004; Levine, 1997). In order to find out if this apoptotic pathway may be involved in the cell death caused by overexpression of PhGFP, I induced PhGFP expression in the eye in a p53 mutant background (see Methods for a detailed description of the crossing scheme). Apoptosis was not abolished when the p53 protein was not present which means that a different apoptotic pathway is used in this case.

\section{III.10 Future extensions of the technique to other PcG complexes}

PcG mediated silencing involves methylation of histone H3. E(Z) (Enhancer of Zeste), one of the members of the isolated PRC2 PcG complex, has a histone methyltransferase activity through its SET domain. In a series of in vitro assays it has been shown that the Drosophila ESC-E(Z) complex as well as the human counterpart EEDEZH2 complex methylate histone H3 at Lysine 27. Methylation on H3K27Me3 facilitates binding of the Pc chromodomain therefore binding of the PRC1 long term repression complex. The methyltransferase activity is lost in vitro when the $\mathrm{E}(\mathrm{Z}) \mathrm{SET}$ domain is mutated (Cao et al., 2002; Czermin et al., 2002) and in vivo it is required for maintenance of repression of homeotic genes (Müller et al., 2002). None of the components of the PRC2 complex has appreciable activity individually (E(Z), Su(Z)12, ESC, Rpd3, p55). E(Z) plus p55 has some MTase activity but the even stronger ability to methylate histone H3 was obtained by adding ESC to E(Z) and p55 (Czermin et al., 2002). Therefore ESC is needed for the methyltransferase activity.

The esc gene has been described previously (Gutjahr et al., 1995). The genomic fragment encodes for a 425 amino acid protein (Fig. 35A) that includes a nuclear localization signal and six tandem repeats of WD-40 domains at its C-terminus. The WD40 repeats are found in a number of eukaryotic proteins that cover a wide variety of functions including adaptor/regulatory modules in signal transduction, pre-mRNA processing, cytoskeleton assembly and cell cycle control. The only common functional theme of WD40 domains is to serve as a stable propeller-like platform to which proteins can bind either stably or reversibly. The ESC protein is localized to the nucleus throughout 
embryogenesis. It is found in all nuclei of cellular blastoderm embryos but during germ band elongation, expression of ESC appears to be restricted to neuroblasts.

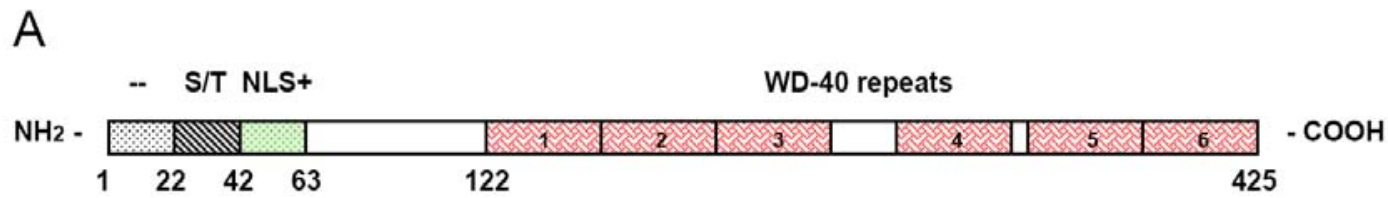

B

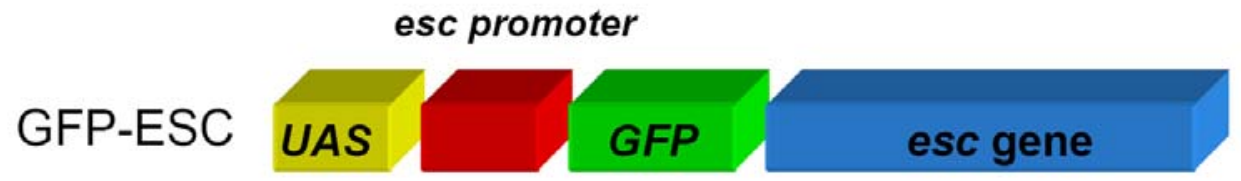

Figure 35. Generation of the GFP-ESC construct. A) Schematic representation of the ESC protein domains. The ESC protein consists of 425 amino acids and includes a stretch of acidic amino acids (--) at its N-terminus (1-22), followed by a Ser/Thr-rich sequence (23-42), a nuclear localization signal (NLS) and basic (+ region (43-63) and six tandem repeats of WD-40 domains at its C-terminus (123-425) (from Gutjahr T, 1995). B) Schematic representation of the GFP-ESC construct.

Later, only a few nuclei in the brain retain ESC protein (Gutjahr et al., 1995). esc mutations cause ectopic expression of homeotic genes at $\sim 6$ hours of development. A temperature sensitive esc mutation demonstrates that esc is critically needed in early embryogenesis during the extended germ band stage (Struhl and Brower, 1982).

To analyze the in vivo kinetic properties of the ESC-E(Z) complex I have generated a GFP-ESC construct. P-element transformants that express full-length ESC with an Nterminal FLAG epitope tag from the esc promoter were capable of completely rescuing all aspects of the phenotype of esc null mutants (Tie et al., 1998) indicating that the FLAG epitope does not interfere with the ESC function. Therefore I chose to tag the GFP moiety to the N-terminal as well and used the esc promoter for induction of ESC-GFP expression. 


\section{DISCUSSION}

The present work is an interdisciplinary study that focuses on the question of how nuclear organization and protein dynamics influence and determine the environmental and mechanistic framework for the gene expression control machinery. A common molecular basis for much of the control of gene expression (whether it occurs at the level of initiation of transcription, RNA processing, translation or RNA transport) is the binding of protein factors (trans-acting factors) to regulatory nucleic acid sequences (cis-acting sequences).

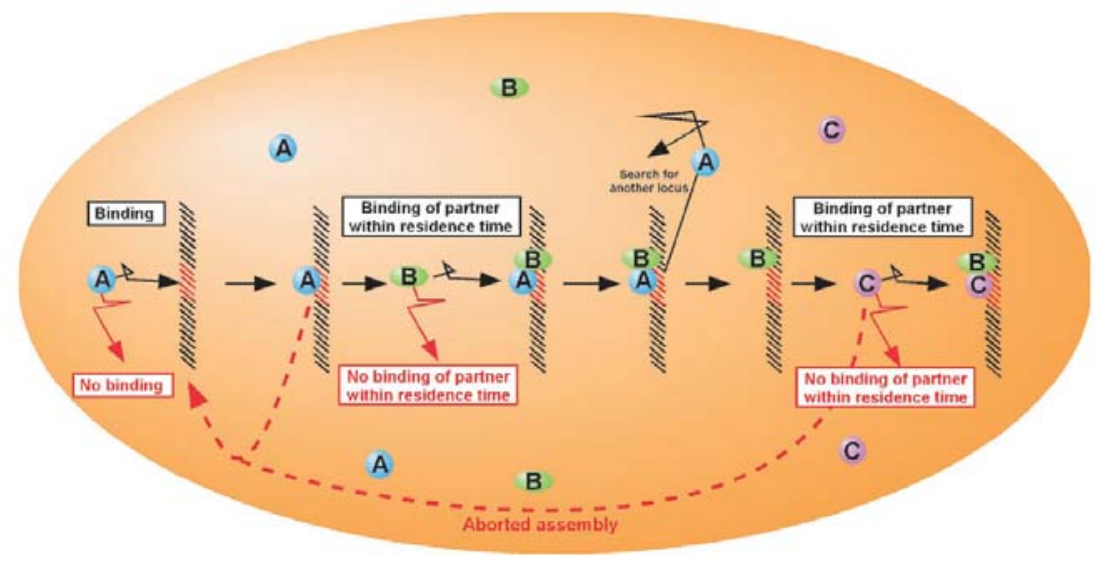

Figure 36. Repeated transient interactions during gene activation. Chromatin remodeling and assembly of the transcription machinery require targeting of numerous components to specific DNA sequences. The rate of transcriptional activation is determined by the availability of any transcription factor at a locus. The random, diffusional mobility of proteins makes remodeling and assembly of the transcription apparatus stochastic. A transcriptional factor A binds transiently to its target sequence. If no binding partner $\mathrm{B}$ is recruited within the residence time of $\mathrm{A}$, B will dissociate prematurely. Analogously, for the next step, $\mathrm{C}$ needs to bind within the residence time of B. The regulator A can either be a transcriptional activator, a cis-regulatory factor, or a component of the transcription machinery, and the binding partners $\mathrm{B}$ and $\mathrm{C}$ may be a chromatin remodeling activity, an interacting cis-regulatory factor, or a second component of the basal transcription machinery, respectively. Cooperative protein-protein interactions and the formation of transcription factories may facilitate the assembly and stabilize assembly intermediates (adapted from Misteli, 2001).

Trans-acting factors are generally organized as multicomponent protein complexes (RNA Polymerases, chromatin remodeling complexes, repression complexes). Studies on the in-vivo behavior of many of these factors and other nuclear components (histones, 
nuclear bodies, see Introduction) reveal that the dynamic properties of proteins are critical for their function (Misteli, 2001). The present view of how these interactions occur and how protein complexes are formed is a stochastic one (Fig. 36). An advantage of such dynamic and probabilistic behavior of proteins in the nucleus is the potential to respond promptly to external cues transmitted by signaling cascades.

If proteins were statically attached to their target sites other energy consuming mechanisms would be needed to release this tight interaction. From the experiments I have described in the preceding sections I conclude that this model is valid also for the Polycomb group repression complex.

\section{IV.1 In vivo experiments to investigate the stability of PcG repression complexes}

In this work I have addressed fundamental questions about the stability and lifetime of PcG repression complexes in living organisms on their target sites. Applying photobleaching microscopy and computer modeling to transgenic Drosophila fly lines expressing GFP fused PcG proteins I was able to measure diffusion constants, dissociation rate constants and residence times of PcG complexes on chromatin. Although complexes of PcG proteins have been isolated in vitro from disrupted cells (Saurin et al., 2001) or assembled from protein components expressed in heterologous systems (Francis et al., 2004), none of these in vitro systems recapitulates the actual repression mechanism occurring in host organisms. That is, specific binding to PREs and gene-specific repression have not been demonstrated in vitro. The in vitro experiments showing chromatin binding and interference with remodeling machines or transcription complexes also demonstrated that individual members of the PRC1 in some cases could produce the same interference as the complete complex (Francis et al., 2004). In addition, repression occurred on chromatin consisting of nucleosomes without histone tails (Shao et al., 1999), results that do not recapitulate the in vivo situation. Removal of cis-acting Polycomb repressor elements (PRE) in vivo even at later developmental stages leads to derepression of homeotic genes (Busturia et al., 1997) indicating that propagation of the silenced state requires the activity of proteins that bind directly to the PREs and not to chromatin in general. In fact, in the organism, deficiency in single PcG genes causes loss of function (Beuchle et al., 2001) and targeting of PcG complexes requires trimethylation of lysine 27 of histone H3 (Cao et al., 
2002; Müller et al., 2002). The discrepancy between in vitro and in vivo repression may be the result of non-physiological conditions for the binding and inhibition experiments. A further complication is the fact that there is evidence of in vivo hierarchical assembly on chromatin (Wang et al., 2004), whereas in vitro complexes are preformed and bound to chromatin. To avoid these problems I chose to address the question of the stability of PcG complexes in live animals and tissues. My results indicate that the repression system is flexible and based on a continuous exchange of the PcG members at specific loci in the genome.

\section{IV.2 Constructs}

Since the original construct of Netter et al. (Netter et al., 2001) containing only the UAS promoter caused overexpression in the salivary gland nuclei, I chose to construct the PhGFP expression vector with both a Pc and an UAS promoter (Brand and Perrimon, 1993) to better control the expression of PhGFP in embryos and larvae The level of $\mathrm{Ph}$ is controlled by feedback inhibition of the Ph promoter (Fauvarque et al., 1995). Both PcG fusion proteins, PcGFP and PhGFP, have been functionally characterized (Dietzel et al., 1999) this work). The PhGFP construct used in this study was able to rescue the null mutant and thus is totally competent in repression. Expression of PcGFP rescued alleles with a mutation in the Pc chromo domain (PcXL5), the domain of the protein that is essential for targeting the PcG complex by binding trimethylated lysine 27 of histone H3. In addition, the PcGFP protein binds to the same polytene chromosome loci as wt Pc (data not shown.) However, mutations in the C-terminal portion of the protein or null mutations $\left(\mathrm{Pc}^{2}, \mathrm{Pc}^{3}\right)$ were not rescued. It is possible that the repression complex cannot accomodate all Pc proteins containing the GFP fusion moiety (see below) or that some other hitherto unknown aspect of Pc protein function is impaired (Dietzel et al., 1999).

\section{IV.3 Photobleaching microscopy in whole organisms}

This is the first photobleaching microscopy study to analyze protein complex stability performed on whole organisms and tissues. As it was described in III.1.2 the technique encountered a number of difficulties due to the non-static nature of the live samples. Most FRAP experiments performed so far in live samples were done in tissue culture cells (Dayel et al., 1999; Dou et al., 2002; McNally et al., 2000; Misteli et al., 2000; 
Stavreva et al., 2004). Cells in culture, especially mammalian cells exhibit incomparably less intracellular mobility. In addition the size of mammalian cells is significantly larger than Drosophila cells (8-10 fold). Conversely, Drosophila cells in embryos and larval tissues showed an extensive reorganization of the nuclear content. More than that, due to developing conditions and cellular divisions that take place in various regions in the embryo, there is a general directional movement of groups of cells especially in the embryos. I concluded that many FRAP techniques developed for use on mammlian tissue culture cells were inadequate for Drosophila system. I have overcome these difficulties by adapting classical photobleaching techniques to conditions offered by Drosophila embryos and tissues.

\section{IV.4 Quantitative FRAP}

The application of fluorescence recovery after photobleaching (FRAP) to fusion proteins of GFP (and its analogs) by confocal microscopy has allowed the study of the dynamics of the steady-state distribution of nuclear proteins in living cells (Houtsmuller and Vermeulen, 2001; Phair and Misteli, 2001; White and Stelzer, 1999). Although most analyses have been qualitative (Cheutin et al., 2003; Dou et al., 2002; Festenstein et al., 2003; Houtsmuller et al., 1999; McNally et al., 2000; Misteli et al., 2000) a quantitative analysis can be used to obtain diffusion and equilibrium constants by the application of combined techniques and appropriate models (Carrero et al., 2003; Dundr et al., 2004; Dundr et al., 2002; Phair et al., 2004; Rabut et al., 2004). In most cases, however, a reaction dominant model has been adopted. That is, because diffusion is faster than most dissociation rates, it is often, incorrectly, neglected in the analyses. Recently, Sprague et al. presented a full analytical treatment for uniformly dispersed binding sites and showed simulations for various boundary conditions and rate constants (Sprague et al., 2004). Because my complexes are not uniformly distributed I have extended this treatment to a model with discrete binding loci that was developed in collaboration with two colleagues in my department, R. Heintzmann and B. Rieger. I present here simulations using this model and diffusion and binding rate constants derived from my experimental data. 


\section{IV.5 3D-iFRAP: a new photobleaching technique}

Our FRAP experiments are somewhat different from those presented previously in the literature. I used live whole animals and tissues rather than adherent tissue culture cells. This fact required adaptation of the acquisition method to include 3-D tracking of loci after inverse FRAP bleaching (3D-iFRAP) since Drosophila diploid cells show an extensive reorganization of nuclear content during measurement times in the minute range (see Fig. 12 and 13). Core histone-GFP that does not dissociate from chromatin in interphase showed similar dynamics, indicating that the movement I see in my cells is not due to dissociation of whole complexes from the chromatin (Post et al., 2005). After registration of the fluorescent locus over time, I was able to calculate the dissociation rate constants for PcG complexes in embryos and imaginal discs. I found very consistent and similar behaviour for both proteins in the complexes in the wing discs, corresponding to dissociation rate constants of $0.032 \pm 0.002 \mathrm{~s}-1$ for PhGFP and $0.034 \pm 0.003 \mathrm{~s}-1$ for PcGFP (Fig. 4 and 5), slightly smaller than in embryos for which the dissociation rate constant for PhGFP was $0.051 \pm 0.004 \mathrm{~s}-1$. All of these complexes were completely exchanged in less than 5 minutes. That is, both PcGFP and PhGFP in PcG complexes in vivo exchanged in an order of magnitude shorter time than the cell cycle. These data rule out a model for repression by which PcG complexes would mask the chromatin and make it inaccessible to transcription factors or other proteins as I discuss below.

\section{IV.6 The influence of diffusion and the amount of unbound protein on FRAP data; determination of rate constants}

\section{IV.6.1 Diffusion processes and binding of PcG proteins to PREs}

As depicted in the model in Fig. 36 availability of proteins is strongly determined by their "roaming" in living cells that is an energy-independent process and likely occurs by a diffusion-based, passive, nondirected mechanism (Kruhlak et al., 2000; Phair and Misteli, 2000). Due to the enormous mass of DNA, RNA, and protein concentrated in the nucleus, one might intuitively think that the nuclear environment is rather viscous and gellike medium. Actually, there is only fourfold difference between the diffusional mobility of branched molecules in the nucleus compared with an aqueous solution (Fushimi and 
Verkman, 1991; Seksek et al., 1997). Photobleaching experiments reveal that high mobility is a general feature of proteins in the cell nucleus independent of the process they are involved in: chromatin organization (Kimura, 2005) and remodeling (Schmiedeberg et al., 2004) transcription activation (Stavreva et al., 2004), pre-mRNA splicing (Dundr et al., 2004; Snaar et al., 2000), rRNA processing (Dundr et al., 2000), telomeric organization (Mattern et al., 2004) or DNA repair (van den Boom et al., 2004).

An important point in studies of Phair and Misteli, 2000, Kruhlak MJ, 2000 as well as in my experience in Drosophila tissues is that GFP alone moves 50 -fold faster than the GFP tagged nuclear proteins, so fast that the photobleached zone could not be imaged before significant recovery. Many other studies have reported (see above) that diffusion of the proteins analyzed is in the range of $0.2-1 \mu \mathrm{m}^{2} \mathrm{~s}^{-1}$. I have shown that the diffusion coefficient of PcG proteins is also around the value of $0.5 \mu \mathrm{m}^{2} \mathrm{~s}^{-1}$. The rather slow diffusion of proteins inside nuclei suggests that molecules undergo transient interactions with nuclear components until they find their specific interaction site where they bind with a shorter or longer residence time according to their binding affinity. The combination of diffusion and high exchange rate ensures targeting of proteins to their site of action by simple diffusion during which proteins effectively scan the nucleus for appropriate binding sites without the requirement for directed targeting, specific signals or signal recognition machinery (Misteli, 2001).

\section{IV.6.2 Determination of dissociation rate constants from FRAP data}

The level of Pc is critical for the maintenance of a competent complex as can be deduced by the fact that $\mathrm{Pc}^{+} /$- heterozygotes show homeotic transformations (Lewis, 1978) and by the destabilization and degradation of Ph in postmitotic cells of Pc3 heterozygotic embryos as shown by antibody binding (Fig. 33). Western blotting revealed that the fusion proteins do not reach levels greater than the wildtype in non-transgenic animals (Table 3). That is, the total PcGFP protein content in the mutants was 0.53 that of wt and PhGFP was comparable to the wt level.

The diffusion constants for PcGFP in early embryos before complex formation $(0.74 \mu \mathrm{m} 2 \mathrm{~s}-1)$ and in the nucleoplasm of salivary gland nuclei $(0.41 \mu \mathrm{m} 2 \mathrm{~s}-1)$ are smaller than one might expect for a protein of $\sim 62 \mathrm{kDa}$, indicating that the protein may exhibit non-specific binding to chromatin. Breiling et al (1999) demonstrated that Pc has an 
affinity for nucleosomes without histone tails and that the $\mathrm{C}$ terminus was critical for this interaction. On the other hand, the Pc chromodomain, essential for complex assembly, has a strong preference for trimethylated Lys 27 over other methylated sites or unmodified H3 showing a KD of $5 \mu \mathrm{M}$ and $>1000 \mu \mathrm{M}$, respectively, in in vitro binding studies (Fischle W. 2003).

As seen in Fig. 28, the amount of free protein obscures the recovery kinetics of the binding process measured on individual bands in salivary glands and must be subtracted prior to fitting the recovery kinetics. From simulations I determined that this effect may be caused by a local depletion of the free pool through the local binding reaction. Such a local depletion in turn slows down the binding reaction and is compensated by the transport of free material by diffusion from distant regions. The latter process is relatively slow, even though the diffusion transport over small distances is fast. Effectively this process of localized depletion and diffusion transport leads to an overall retardation of the recovery times. Computer simulation of FRAP curves using values for the diffusion constants determined experimentally showed that diffusion plays an important role even in the case of PhGFP, which is much less abundant in the nucleoplasm as a free protein. I created masks in the images to separate out pixels that contained predominantly non-bound protein from that involved in complexes. By first fitting and subtracting the diffusion component I was able to fit the resulting recovery curves to a single exponential, as in a kinetic process in a standard chemical equilibrium (Fig. 30). The dissociation rate constants were in the same range as those measured in the $2 \mathrm{~N}$ cells of embryos and imaginal discs but the means were shifted towards a value $\sim 1 / 3$ that for PhGFP to $\sim 1 / 4$ of that for PcGFP. These differences could reflect some unintended bias in the selection of the bleach loci in either the $2 \mathrm{~N}$ nuclei or the polytene bands. On the other hand, each polytene band represents thousands of complexes at one or a few PREs rather than an average of many different complexes and thus, these data may be more robust. In either case, even when PREs are in close proximity, such as is the case of several thousand chromatids closely aligned in the polytene chromosomes, the PcG proteins are in a chemical equilibrium with unbound protein. The reduced rate constants may reflect the large local binding sites whereby a dissociated protein does not immediately join the "free pool” but has a higher probability to rebind in the close vicinity. On the other hand the proteins are not "trapped" in the complex but rather are able to completely exchange in less than 6 minutes. The reproducibility of the recovery times of individual bands subjected to successive FRAP 
measurements is shown in Fig. 29B indicating that the differences of 2 to 3 fold in recovery times (Fig. 31) between different bands can be considered reproducible and significant.

The t0 values calculated from these recovery curves are, however, not a direct measure of residence time because of their dependence on the effect of diffusion transport in combination with ongoing depletion from the free pool. As could be demonstrated using simulations, if the dissociation rate constant and the concentration of free protein were the same for all complexes then one would expect the recovery rate to depend linearly on the amount of bound protein (lines in Fig. 31B), which is essentially a measure of the capability of a spot to deplete the free protein pool during recovery. As seen in the same figure the experimental data do not show such a correlation, implying that there are differences in the composition of the complexes on different genetic loci and that the dissociation rate constants, though similar (within a factor of 5, Fig. 32A), reflect the specific mixture of PcG and non-PcG auxiliary proteins on the polytene bands. Such an interpretation is compatible with the data of Rastelli et al. who showed varying occupancy of PcG proteins and Zeste on more than 100 bands by immunohistochemistry on polytene chromosomes (Rastelli et al., 1993). To rule out the possibility of a very slow component which would appear as an immobile fraction in a single exponential fit I also fitted the data with a sum of 2 exponentials, but did not find a consistent second time in this case and less precision of the first time. Thus, I conclude that both PcGFP and PhGPF in repression complexes exchange within a few minutes in live Drosophila cells.

\section{IV.6.3 Determination of pseudo-association rate constants from the equilibrium situation}

In Fig. 32B the pseudo-association rate constants as described in the section II.3.2.2 are plotted for PhGFP. The values are an order of magnitude larger than the dissociation rate constants and thus dissociation is rate-limiting. I have not attempted to present pseudoassociation rate constants for PcGFP for the following reasons. I assume that the number of binding sites for $\mathrm{Pc}$ and $\mathrm{Ph}$ are approximately equal since the proteins bind to overlapping sites on polytene chromosomes (Rastelli et al., 1993), isolated complexes of the proteins contain equimolar quantities of both proteins (Saurin et al., 2001), and they are targeted to the same PREs by ChIP analysis (Breiling et al., 2001). As discussed above, the off-rates 
are similar for the two proteins. However I can see a larger pool of unbound PcGFP compared to PhGFP in both embryos and larval tissue, Figs. 11 and 22. As determined from Western blots in salivary glands PcGFP is not present in amounts higher than PhGFP that is to saturate the binding sites (Fig. 11K). Wang et al (2004) have shown that there is sequential recruitment of PcG complexes to the PREs whereby Pc targets the complex to chromatin by binding to trimethylated H3K27 (Cao et al., 2002; Czermin et al., 2002; Fischle et al., 2003; Wang et al., 2004). From these considerations, I postulate that the PcGFP fusion protein, although competent to target PREs with modified histones and engage in a competent complex, cannot substitute for all Pc molecules in the complexes (perhaps due to steric hinderance of adjacent GFP moieties). My data suggest that the unmodified Pc is preferred in the complex by a factor of about 4-5 and thus association rate constants calculated for PcGFP will not properly reflect the true on rate of the unmodified protein whereas off rates should not be adversely affected.

I calculated the ratio of the bound/free fusion proteins from the segmentation of the salivary gland prebleach FRAP images to be 1:10 and from the western blots (Fig. 11) the ratio of the fusion protein to wt protein of 1:1. I estimated the absolute concentration of GFP protein in the salivary gland nuclei to be $\sim 2-4 \mu \mathrm{M}$ by comparison to the intensity of droplets of purified GFP protein in an immiscible solution in our microscope system. If the total concentration of binding sites is equivalent to the concentration of the bound $\mathrm{Ph}$ I can estimate the $\mathrm{K}_{\mathrm{D}}$ for the protein in vivo in salivary gland nuclei to be $\sim 5 \mu \mathrm{M}$.

\section{IV.7 Simulation of diffusion and binding reactions}

To test the validity of the chemical model generated for the binding of Polycomb group of proteins to their target sites I have used computer simulation programs developed in collaboration with theoreticians. This allowed me to interpret the results from photobleaching experiment more accurately and to obtain quantitative data on the binding kinetics of PcG proteins. Moreover computer simulations resulted in a better understanding of biophysical processes underlying FRAP that are not detected in experiments on biological samples. FRAP experiments in vivo are 3 dimensional processes involving a particular cone-like shape of the laser profile while bleaching and diffusion of molecules in a volume. The simulation is designed in two dimensions but the model can be extended for 
a three dimensional situation in which all planes contain identical concentrations of all involved substances and boundary conditions ('cylindrical' symmetry).

Biophysical techniques like FCS (fluorescence correlation spectroscopy) or photobleaching microscopy are means to measure diffusion coefficients. In contrast to FRAP in FCS there is no need to photobleach the sample. FCS is essentially a photoncounting technique that monitors the fluorescent signal emanating from a very small optically defined volume. The residence time of the molecule in the observation volume can be used to determine the diffusion coefficient for the fluorescent-labeled proteins in the volume (Berland KM, 2004). In my experiments I used only FRAP to determine the diffusion coefficient of PcG proteins. Due to the small size of nuclei compared to mammalian cells, there was a need to understand whether there are limits of FRAP in such conditions. Simulating FRAP with various nuclear sizes, I observed that for the same bleach box size, the smaller the nucleus was, the more the diffusion coefficient deviated from the theoretical curve. Crucial in measuring diffusion coefficients by FRAP is the ratio bleach box/ nucleus size. Axelrod's equation (Axelrod et al., 1976) is valid if the ratio of the bleach box size/nucleus size is $1 / 10$. Smaller ratios require a correction in $\gamma_{D}$. factor (equation 3) (Fig. 15). Choosing a big bleach box is generally advantageous since this improves the amount of measured fluorophores and thus the signal to noise ratio. In addition the measured recovery curve can be expected to be slower thus also leading to a more precise estimate when the maximal frame-rate of the microscope is too slow to accurately capture the fast recovery in the small box. As explained above the bleach box size should nevertheless stay below $1 / 10$ of the nucleus size to make the original theory of Axelrod applicable. However, in small Drosophila nuclei such a compromise is not feasible. Therefore modified $\gamma_{D}$ values had to be computed in order to use Axelrod's expression.

When simulating binding reactions shown in Fig. 25 I sought to reproduce the process of binding of PcG proteins to particular loci in the genome. Therefore I created a situation in silico where the 2-dimensional nucleus had the size of a typical salivary gland nucleus, the diffusion coefficient was set to the value I had measured, the range of concentration of binding sites was comparable to the loci seen in confocal images, the amount of bleaching was $80 \%$, similar to that achieved in the real FRAP, and the presence of other loci was represented by a single intense locus. One of the major observations was that the amount of unbound protein influences significantly the binding reactions. For the 
binding reaction to occur unperturbed (with the dissociation constant characteristic for the particular locus, that depends on the affinity of the protein to the binding sites and other proteins present in the complex) there must be sufficient level of unbound protein in the nucleus, i.e. $>\sim 30 \%$ of the total fluorescent intensity. The amount of PcG proteins bound is less than $10 \%$ therefore there is sufficient unbound material therefore this is not a limiting factor in the recovery process. Another observation was that the value of the diffusion coefficient is also a critical factor for the binding process. Two loci with different intensities and the same dissociation constant will have different recovery times when diffusion occurs with the speed I measured experimentally $\left(0.5 \mu \mathrm{m}^{2} \mathrm{~s}^{-1}\right)$. The more concentrated the locus in binding sites is the longer the recovery process will last. This would not occur if molecules diffused infinitely fast in the nucleus.

All these results provided by the simulation experiments assisted in understanding and interpreting the photobleaching microscopy and the complicated biophysical processes underlying it. The set of models and simulations developed as an outgrowth of this thesis will help to design future FRAP experiments with a better accuracy and interpret data more precisely. They allowed me to determine the kinetic properties of PcG proteins and to confidently determine the heterogeneity of these complexes at individual PREs. 


\section{SUMMARY AND CONCLUSIONS}

Using photobleaching in confocal microscopy and computer simulations I determined the stability of PcG protein complexes during development. These are the first FRAP experiments performed in whole live organisms and tissues. I found that all complexes were exchangeable throughout all developmental stages. The relatively short residence times of 2- 6 minutes for $\mathrm{Ph}$ and $\mathrm{Pc}$ in the repression complex rule out models for repression that invoke blocking chromatin access. They also suggest that competition could exist between antagonistic factors at PREs and promoters allowing modulation of the state of repression during development by changes in their balance. These data complement those found in other systems such as the direct competition for chromatin binding sites between histone $\mathrm{H} 1$ and microinjected high-mobility group (HMG) proteins as demonstrated by Catez et al. (Catez et al., 2004).

Most FRAP studies of nuclear proteins have involved components in transcription complexes or transcriptional activators that exchange in less than 2 minutes (Phair et al., 2004). The only repressor protein that has previously been investigated is heterochromatin protein 1 (HP1), a protein targeted to heterochromatin in higher eukaryotes (Cheutin et al., 2003; Festenstein et al., 2003). Although HP1 is loaded directly onto the chromatin during replication it was found by FRAP to bind only transiently to chromatin with a maximum residence time of $\sim 60$ seconds. Thus, both HP1 and PcG repression complexes appear to function by dynamic competition with other chromatin-binding proteins rather than by formation of a static, higher-order chromatin structure with immobilized bound repressors. My FRAP measurements on polytene chromosomes revealed differences in the dissociation rate constants between individual bands that imply a flexible repression system of complexes with various compositions that influence the binding affinity of other members and whose turnover is in the order of a few minutes.

I conclude that (i) activation and repression can be dynamically controlled by simple chemical equilibria; (ii) reduction in PcG levels will facilitate epigenetic change and may explain why non-cycling cells can be reprogrammed more easily than cycling cells (Baxter et al., 2004); (iii) PcG complexes are exchangeable protein assemblies maintaining repression over many cell cycles by simple chemical equilibria. 


\section{REFERENCES}

Axelrod, D., Koppel, D. E., Schlessinger, J., Elson, E. and Webb, W. W. (1976). Mobility measurement by analysis of fluorescence photobleaching recovery kinetics. Biophysical Journal 16, 1055-1069.

Beuchle, D., Struhl, G. and Muller, J. (2001). Polycomb group proteins and heritable silencing of Drosophila Hox genes. Development 128, 993-1004.

Bienz, M. and Muller, J. (1995). Transcriptional silencing of homeotic genes in Drosophila. Bioessays 17, 775-784.

Brand, A. H. and Perrimon, N. (1993). Targeted gene expression as a means of altering cell fates and generating dominant phenotypes. Development 118, 401-415.

Breiling, A., O'Neill, L. P., D'Eliseo, D., Turner, B. M. and Orlando, V. (2004). Epigenome changes in active and inactive polycomb-group-controlled regions. EMBO Rep 5, 976-82.

Buchenau, P., Hodgson, J., Strutt, H. and Arndt-Jovin, D. J. (1998). The distribution of polycomb-group proteins during cell division and development in Drosophila embryos: impact on models for silencing. Journal of Cell Biology 141, 469-481.

Busturia, A. and Morata, G. (1988). Ectopic expression of homeotic genes caused by the elimination of the Polycomb gene in Drosophila imaginal epidermis. Development 104, 713-720.

Busturia, A., Wightman, C. D. and Sakonju, S. (1997). A silencer is required for maintenance of transcriptional repression throughout Drosophila development.

Development 124, 4343-4350.

Cao, R., Wang, L., Wang, H., Xia, L., Erdjument-Bromage, H., Tempst, P., Jones, R. S. and Zhang, Y. (2002). Role of histone H3 lysine 27 methylation in Polycomb-group silencing. Science 298, 1039-1043.

Capdevila, J. and Guerrero, I. (1994). Targeted expression of the signaling molecule Decapentaplegic Induces pattern duplications and growth alterations in Drosophila wings. EMBO J. 13, 4459-4468.

Carrero, G., McDonald, D., Crawford, E., de Vries, G. and Hendzel, M. J. (2003). Using FRAP and mathematical modeling to determine the in vivo kinetics of nuclear proteins. Methods 29, 14-28.

Catez, F., Yang, H., Tracey, K. J., Reeves, R., Misteli, T. and Bustin, M. (2004). Network of Dynamic Interactions between Histone H1 and High-Mobility-Group Proteins in Chromatin. Molecular and Cellular Biology 24, 4321-4328. 
Chan, C. S., Rastelli, L. and Pirrotta, V. (1994). A Polycomb response element in the Ubx gene that determines an epigenetically inherited state of repression. EMBO Journal 13, 2553-2564.

Chiang, A., O'Connor, M. B., Paro, R., Simon, J. and Bender, W. (1995). Discrete Polycomb-binding sites in each parasegmental domain of the bithorax complex.

Development 121, 1681-1689.

Cremer, T. and Cremer, C. (2001). Chromosome territories, nuclear architecture and gene regulation in mammalian cells. 2 4, 292-301.

Czermin, B., Melfi, R., McCabe, D., Seitz, V., Imhof, A. and Pirrotta, V. (2002). Drosophila Enhancer of Zeste/ESC complexes have a histone H3 methyltransferase activity that marks chromosomal polycomb sites. Cell 111, 185-196.

Dayel, M. J., Hom, E. F. Y. and Verkman, A. S. (1999). Diffusion of Green Fluorescent Protein in the Aqueous-Phase Lumen of Endoplasmic Reticulum. Biophysical Journal 76, 2843-2851.

Dellino, G. I., Schwartz, Y. B., Farkas, G., McCabe, D., Elgin, S. C. R. and Pirrotta, V. (2004). Polycomb silencing blocks transcription initiation. Molecular Cell 13, 887-893.

Dietzel, S., Niemann, H., Brückner, B., Maurange, C. and Paro, R. (1999). The nuclear distribution of Polycomb during Drosophila melanogaster development shown with a GFP fusion protein. Chromosoma 108, 83-94.

Dou, Y., Bowen, J., Liu, Y. and Gorovsky, M. A. (2002). Phosophorylation and an ATPdependent process increase the dynamic exchange of $\mathrm{H} 1$ in chromatin. Journal of Cell Biology 158, 1161-1170.

Dundr, M., Herbert, M. D., Karpova, T. S., Stanek, D., Xu, H., Shpargel, K. B., Meier, U. T., Neugebauer, K. M., Matera, A. G. and Misteli, T. (2004). In vivo kinetics of Cajal body components. Journal of Cell Biology 164, 831-842.

Dundr, M., Hoffmann-Rohrer, U., Hu, Q., Grummt, I., Rothblum, L. I., Phair, R. D. and Misteli, T. (2002). A kinetic framework for a mammalian RNA polymerase in vivo. Science 298, 1623-1626.

Dundr, M., Misteli, T. and Olson, M. O. J. (2000). The Dynamics of Postmitotic Reassembly of the Nucleolus. Journal of Cell Biology 150, 433-446.

Dura, J. M., Randsholt, N. B., Deatrick, J., Erk, I., Santamaria, P., Freeman, J. D., Freeman, S. J., Weddell, D. and Brock, H. W. (1987). A complex genetic locus, polyhomeotic, is required for segmental specification and epidermal development in D. melanogaster. Cell 51, 829-839.

Edidin, M., Zagyansky, Y. and Lardner, T. J. (1976). Measurement of membrane protein lateral diffusion in single cells. Science 191, 466-468. 
Fauvarque, M. O. and Dura, J. M. (1993). Polyhomeotic regulatory sequences induce developmental regulator-dependent variegation and targeted P-element insertions in Drosophila. Genes and Development 7, 1508-1520.

Fauvarque, M. O., Zuber, V. and Dura, J. M. (1995). Regulation of polyhomeotic transcription may involve local changes in chromatin activity in Drosophila. Mechanisms of Development 52, 343-355.

Feinberg, A. P., Oshimura, M. and Barrett, J. C. (2002). Epigenetic Mechanisms in Human Disease. Cancer Research 62, 6784-6787.

Feinberg, A. P. and Vogelstein, B. (1983). Hypomethylation distinguishes genes of some human cancers from their normal counterparts. Nature 301, 89-92.

Felsenfeld, G. and Groudine, M. (2003). Controlling the double helix. Nature 421, 448.

Fitzgerald, D. P. and Bender, W. (2001). Polycomb group repression reduces DNA accessibility. Molecular and Cellular Biology 21, 6585-6597.

Francis, N. J. and Kingston, R. E. (2001). Mechanisms of transcriptional memory. Nat Rev Mol Cell Biol 2, 409-421.

Francis, N. J., Kingston, R. E. and Woodcock, C. (2004). Chromatin compaction by a Polycomb Group protein complex. Science 306, 1574-1577.

Franke, A., DeCamillis, M., Zink, D., Cheng, N., Brock, H. W. and Paro, R. (1992). Polycomb and polyhomeotic are constituents of a multimeric protein complex in chromatin of Drosophila melanogaster. EMBO Journal 11, 2941-2950.

Fushimi, K. and Verkman, A. S. (1991). Low viscosity in the aqueous domain of cell cytoplasm measured by picosecond polarization microfluorimetry. Journal of Cell Biology 112, 719-725.

Garcia-Bellido, A., PRipoll, P. and Morata, G. (1976). Developmental compartmentalization in the dorsal mesothoracic disc of Drosophila. Dev Biol. 48, 132147.

Gaul, U. and Jäckle, H. (1989). Analysis of maternal effect mutant combinations elucidates regulation and function of the overlap of hunchback and Krüppel gene expression in the Drosophila blastoderm embryo. Development 107, 651-662.

Gilbert, N., Boyle, S., Fiegler, H., Woodfine, K., Carter, N. P. and Bickmore, W. A. (2004). Chromatin Architecture of the Human Genome: Gene-Rich Domains Are Enriched in Open Chromatin Fibers. Cell 118, 555.

Grewal, S. I. and Moazed, D. (2003). Heterochromatin and epigenetic control of gene expression. Science 301, 798-802.

Gutjahr, T., Frei, E., Spicer, C., Baumgartner, S., White, R. A. and Noll, M. (1995). The Polycomb-group gene, extra sex combs, encodes a nuclear member of the WD-40 repeat family. EMBO Journal 14, 4296-4306. 
Hernández, G., Lalioti, V., Vandekerckhove, J., Sierra, J. M. and Santarén, J. F. (2004). Identification and characterization of the expression of the translation initiation factor 4A (eIF4A) from Drosophila melanogaster. Proteomics 4, 316 - 326.

Houtsmuller, A. B. and Vermeulen, W. (2001). Macromolecular dynamics in living cell nuclei revealed by fluorescence redistribution after photobleaching. Histochemistry and Cell Biology 115, 13-21.

Ingham, P. W. (1985). A clonal analysis of the requirement for the trithorax gene in the diversification of segments in Drosophila. Journal of Embryology and Experimental Morphology 89, 349-365.

Jacobson, K., Derzko, Z., Wu, E. S., Hou, Y. and Poste, G. (1976). Measurement of the lateral mobility of cell surface components in single, living cells by fluorescence recovery after photobleaching. Journal of Supramolecular Structure 5, 565-576.

Kennison, J. A. and Tamkun, J. W. (1988). Dosage-dependent modifiers of polycomb and antennapedia mutations in Drosophila. Proceedings of the National Academy of Sciences of the United States of America 85, 8136-8140.

Kimura, H. (2005). Histone dynamics in living cells revealed by photobleaching. DNA Repair In Press, Corrected Proof.

Kruhlak, M. J., Lever, M. A., Fischle, W., Verdin, E., Bazett-Jones, D. P. and Hendzel, M. J. (2000). Reduced Mobility of the Alternate Splicing Factor (ASF) through the Nucleoplasm and Steady State Speckle Compartments. Journal of Cell Biology 150, 41-52.

Kuzmichev, A., Nishioka, K., Erdjument-Bromage, H., Tempst, P. and Reinberg, D. (2002). Histone methyltransferase activity associated with a human multiprotein complex containing the Enhancer of Zeste protein. Genes and Development 16, 2893-905.

Lavigne, M., Francis, N. J., King, I. F. G. and Kingston, R. E. (2004). Propagation of silencing: Recruitment and repression of naive chromatin - In trans by polycomb repressed chromatin. Molecular Cell 13, 415-425.

Levine, A. J. (1997). p53, the cellular gatekeeper for growth and division. Cell 88, 323331.

Levine, S. S., King, I. F. G. and Kingston, R. E. (2004). Division of labor in Polycomb group repression. Trends in Biochemical Sciences 29, 478-485.

Levine, S. S., Weiss, A., Erdjument-Bromage, H., Shao, Z., Tempst, P. and Kingston, R. E. (2002). The core of the polycomb repressive complex is compositionally and functionally conserved in flies and humans. Molecular and Cellular Biology 22, 6070-8.

Lewis, E. B. (1963). Genes and developmental pathways. Am Zool. 3, 33-56.

Lewis, E. B. (1978). A gene complex controlling segmentation in Drosophila. Nature 276, 565-570. 
Lippincott-Schwartz, J., Altan-Bonnet, N. and Patterson, G. H. (2003). Photobleaching and photoactivation: following protein dynamics in living cells. Nat. Cell Biol., S7-S14.

Lippincott-Schwartz, J. and Patterson, G. H. (2003). Development and Use of Fluorescent Protein Markers in Living Cells. Science 300, 87-91.

Luby-Phelps, K. (2000). Cytoarchitecture and physical properties of cytoplasm: volume, viscosity, diffusion, intracellular surface area. 192, 189-221.

Mattern, K. A., Swiggers, S. J. J., Nigg, A. L., Lowenberg, B., Houtsmuller, A. B. and Zijlmans, J. M. J. M. (2004). Dynamics of Protein Binding to Telomeres in Living Cells: Implications for Telomere Structure and Function. Molecular and Cellular Biology 24, 5587-5594.

McNally, J. G., Müller, W. G., Walker, D., Wolford, R. and Hager, G. L. (2000). The glucocorticoid receptor: rapid exchange with regulatory sites in living cells. Science 287, 1262-1265.

Min, J., Zhang, Y. and Xu, R. M. (2003). Structural basis for specific binding of Polycomb chromodomain to histone H3 methylated at Lys 27. Genes and Development 17, 1823-8.

Misteli, T. (2001). Protein dynamics: Implications for nuclear architecture and gene expression. Science 291, 843-847.

Misteli, T. (2005). Concepts in nuclear architecture. Bioessays 27, 477-487.

Misteli, T., Gunjan, A., Hock, R., Bustin, M. and Brown, D. T. (2000). Dynamic binding of histone H1 to chromatin in living cells. Nature 408, 877-881.

Mulholland, N. M., King, I. F. G. and Kingston, R. E. (2003). Regulation of Polycomb roup complexes by the sequence-specific DNA binding proteins Zeste and GAGA. Genes and Development 17, 2741-2746.

Muller, J. and Bienz, M. (1991). Long range repression conferring boundaries of Ultrabithorax expression in the Drosophila embryo. EMBO Journal 10, 3147-3155.

Müller, J., Hart, C. M., Francis, N. J., Vargas, M. L., Sengupta, A., Wild, B., Miller, E. L., O'Connor, M. B., Kingston, R. E. and Simon, J. A. (2002). Histone methyltransferase activity of a Drosophila Polycomb group repressor complex. Cell 111, 197-208.

Nedelec, F. (2002). Computer simulations reveal motor properties generating stable antiparallel microtubule interactions. Journal of Cell Biology 158, 1005-1015.

Netter, S., Faucheux, M. and Theodore, L. (2001). Developmental dynamics of a polyhomeotic-EGFP fusion in vivo. DNA and Cell Biology 20, 483-492.

Ng, J., Hart, C. M., Morgan, K. and Simon, J. A. (2000). A Drosophila ESC-E(Z) protein complex is distinct from other polycomb group complexes and contains covalently modified ESC. Mol Cell Biol. 20, 3069-3078. 
Orlando, V. (2003). Polycomb, epigenomes, and control of cell identity. Cell 112, 599606.

Orlando, V., Jane, E. P., Chinwalla, V., Harte, P. J. and Paro, R. (1998). Binding of trithorax and Polycomb proteins to the bithorax complex: dynamic changes during early Drosophila embryogenesis. EMBO Journal 17, 5141-5150.

Orphanides, G. and Reinberg, D. (2002). A Unified Theory of Gene Expression. Cell 108, 439.

Parada, L. A. and Misteli, T. (2002). Chromosome positioning in the interphase nucleus. Trends in Cell Biology 12, 425.

Paro, R. and Hogness, D. S. (1991). The Polycomb protein shares a homologous domain with a heterochromatin-associated protein of Drosophila. Proc Natl Acad Sci USA 88, 263267.

Peters, R., Peters, J., Tews, K. H. and Bahr, W. (1974). A microfluorimetric study of translational diffusion in erythrocyte membranes. Biochimica et Biophysica Acta 367, 282294.

Phair, R. D. and Misteli, T. (2000). High mobility of proteins in the mammalian cell nucleus. Nature 404, 604-608.

Phair, R. D. and Misteli, T. (2001). Kinetic modeling approaches to in vivo imaging. Nat Rev Mol Cell Biol 2, 898-907.

Phair, R. D., Scaffidi, P., Elbi, C., Vacerova, J., Dey, A., Ozato, K., Brown, D. T., Hager, G., Bustin, M. and Misteli, T. (2004). Global nature of dynamic proteinchromatin interactions in vivo: three dimensional genome scanning and dynamic interaction networks of chromatin proteins. Molecular and Cellular Biology 24, 63936402.

Post, J. N., Liedke, K. A., Rieger, B. and Arndt-Jovin, D. J. (2005). One- and twophoton photoactivation of a paGFP-fusion protein in live Drosophila embryos. FEBS Letters 579, 325-330.

Rabut, G., Doye, V. and Ellenberg, J. (2004). Mapping the dynamic organization of the nuclear pore complex inside single living cells. Nat Cell Biol 6, 1114.

Rastelli, L., Chan, C. S. and Pirrotta, V. (1993). Related chromosome binding sites for zeste, suppressors of zeste and Polycomb group proteins in Drosophila and their dependence on Enhancer of zeste function. EMBO Journal 12, 1513-1522.

Ringrose, L., Ehret, H. and Paro, R. (2004). Distinct Contributions of Histone H3 Lysine 9 and 27 Methylation to Locus-Specific Stability of Polycomb Complexes. Molecular Cell 16, 641 .

Ringrose, L., Rehmsmeier, M., Dura, J. M. and Paro, R. (2003). Genome-wide prediction of Polycomb/Trithorax response elements in Drosophila melanogaster. Developmental Cell 5, 759-771. 
Rong, Y. S., Titen, S. W., Xie, H. B., Golic, M. M., Bastiani, M., Bandyopadhyay, P., Olivera, B. M., Brodsky, M., Rubin, G. M. and Golic, K. G. (2002). Targeted mutagenesis by homologous recombination in D. melanogaster. Genes and Development 16, 1568-1581.

Sanchez-Herrero, E., Vernos, I., Marco, R. and Morata, G. (1985). Genetic organization of Drosophila bithorax complex. Nature 313, 108-113.

Saurin, A. J., Shao, Z., Erdjument-Bromage, H., Tempst, P. and Kingston, R. E. (2001). A Drosophila Polycomb group complex includes ZESTE and dTAFII proteins. Nature 412, 655-60.

Schmiedeberg, L., Weisshart, K., Diekmann, S., Meyer zu Hoerste, G. and Hemmerich, P. (2004). High- and Low-mobility Populations of HP1 in Heterochromatin of Mammalian Cells. Molecular Biology of the Cell 15, 2819-2833.

Seksek, O., Biwersi, J. and Verkman, A. S. (1997). Translational Diffusion of Macromolecule-sized Solutes in Cytoplasm and Nucleus. Journal of Cell Biology 138, 131-142.

Sengel, P. and Mandaron, P. (1969). Aspects morphologiques du développement in vitro des disques imaginaux de la Drosophila. Wilhelm Roux' Archiv 174, 303-311.

Shao, Z. H., Raible, F., Mollaaghababa, R., Guyon, J. R., Wu, C. T., Bender, W. and Kingston, R. E. (1999). Stabilization of chromatin structure by PRC1, a Polycomb complex. Cell 98, 37-46.

Simon, J., Chiang, A., Bender, W., Shimell, M. J. and O'Connor, M. (1993). Elements of the Drosophila Bithorax Complex That Mediate Repression by Polycomb Group Products. Developmental Biology 158, 131.

Simon, J. A. and Tamkun, J. W. (2002). Programming off and on states in chromatin: mechanism of Polycomb and trithorax group complexes. Current Opinion in Genetics and Development 12, 210-218.

Snaar, S., Wiesmeijer, K., Jochemsen, A. G., Tanke, H. J. and Dirks, R. W. (2000). Mutational Analysis of Fibrillarin and Its Mobility in Living Human Cells. Journal of Cell Biology 151, 653-662.

Spradling, A. C. and Rubin, G. M. (1982). Transposition of cloned P elements into Drosophila germ line chromosomes. Science 218, 341-347.

Sprague, B. L., Pego, R. L., Stavreva, D. A. and McNally, J. G. (2004). Analysis of binding reactions by fluorescence recovery after photobleaching. Biophysical Journal 86, 3473-3495.

Stavreva, D. A., Müller, W. G., Hager, G. L., Smith, C. L. and McNally, J. G. (2004). Rapid glucocorticoid receptor exchange at a promoter is coupled to transcription and regulated by chaperones and proteasomes. Molecular and Cellular Biology 24, 2682-2697. 
Struhl, G. and Brower, D. (1982). Early role of the esc + gene product in the determination of segments in Drosophila. Cell 31, 285-292.

Strutt, H., Cavalli, G. and Paro, R. (1997). Co-localization of Polycomb protein and GAGA factor on regulatory elements responsible for the maintenance of homeotic gene expression. EMBO Journal 16, 3621-3632.

Strutt, H. and Paro, R. (1997). The Polycomb group protein complex of Drosophila melanogaster has different compositions at different target genes. Molecular and Cellular Biology 17, 6773-6783.

Tautz, D. (1988). Regulation of the Drosophila segmentation gene hunchback by two maternal morphogenetic centres. Nature 332, 281-284.

Tie, F., Furuyama, T. and Harte, P. J. (1998). The Drosophila Polycomb Group proteins ESC and E(Z) bind directly to each other and co-localize at multiple chromosomal sites. Development 125, 3483-3496.

van den Boom, V., Citterio, E., Hoogstraten, D., Zotter, A., Egly, J.-M., van Cappellen, W. A., Hoeijmakers, J. H. J., Houtsmuller, A. B. and Vermeulen, W. (2004). DNA damage stabilizes interaction of CSB with the transcription elongation machinery. Journal of Cell Biology 166, 27-36.

Verkman, A. S. (2002). Solute and macromolecule diffusion in cellular aqueous compartments. Trends in Biochemical Sciences 27, 27-33.

Vermaak, D., Ahmad, K. and Henikoff, S. (2003). Maintenance of chromatin states:an open-and-shut case. Current Opinion in Cell Biology 15, 266-274.

Wang, L., Brown, J. L., Cao, R., Zhang, Y., Kassis, J. A. and Jones, R. S. (2004). Hierarchical recruitment of Polycomb group silencing complexes. Molecular Cell 14, 637646.

White, J. and Stelzer, E. (1999). Photobleaching GFP reveals protein dynamics inside live cells. Trends in Cell Biology 9, 61-65.

Yamamoto, K., Sonoda, M., Inokuchi, J., Shirasawa, S. and Sasazuki, T. (2004). Polycomb group suppressor of zeste 12 links heterochromatin protein 1 alpha and enhancer of zeste 2. Journal of Biological Chemistry 279, 401-406.

Zink, B. and Paro, R. (1989). In vivo binding pattern of a trans-regulator of homoeotic genes in Drosophila melanogaster. Nature 337, 468-471. 


\section{APPENDIX}

\section{VII.1 Grid based diffusion and binding simulation}

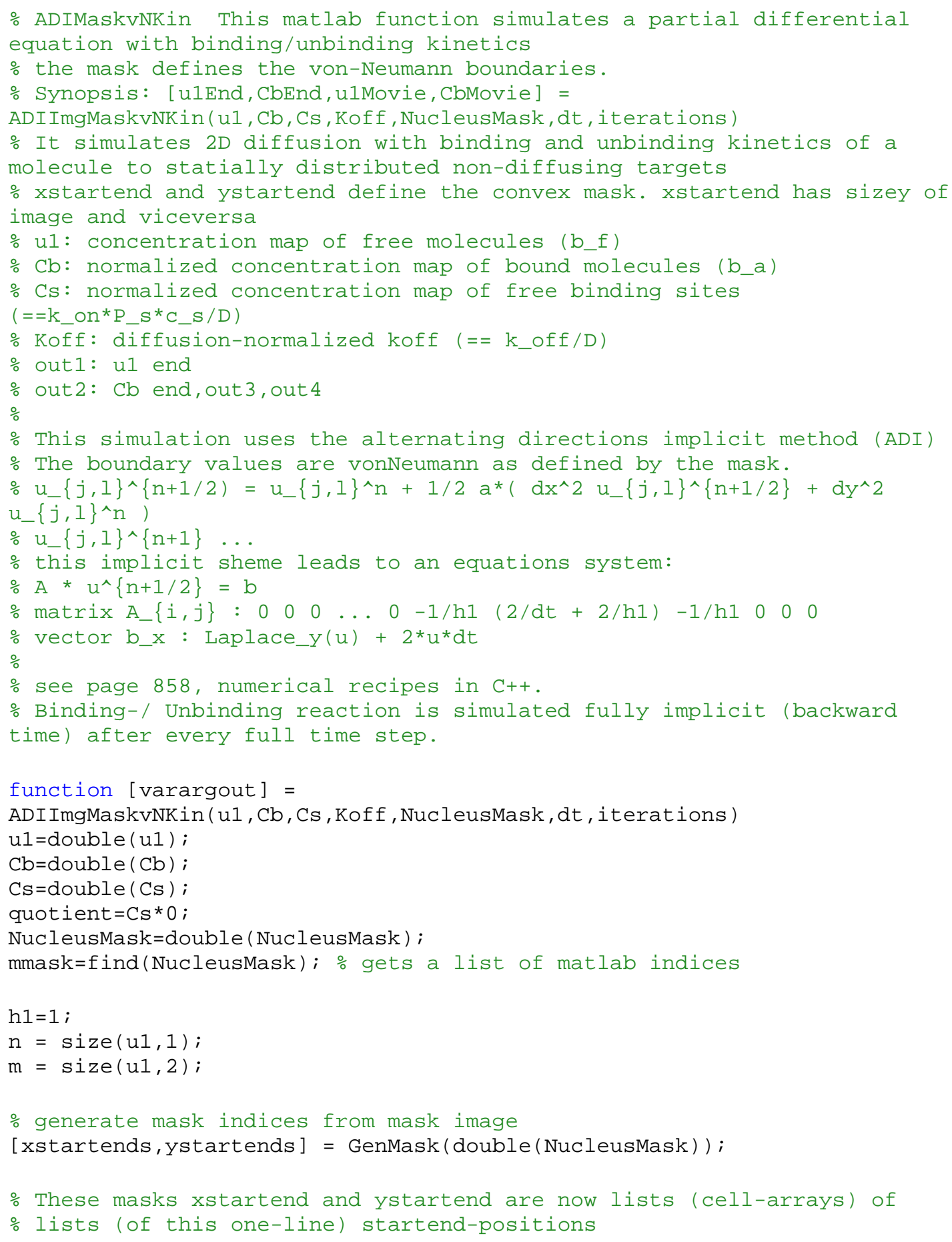




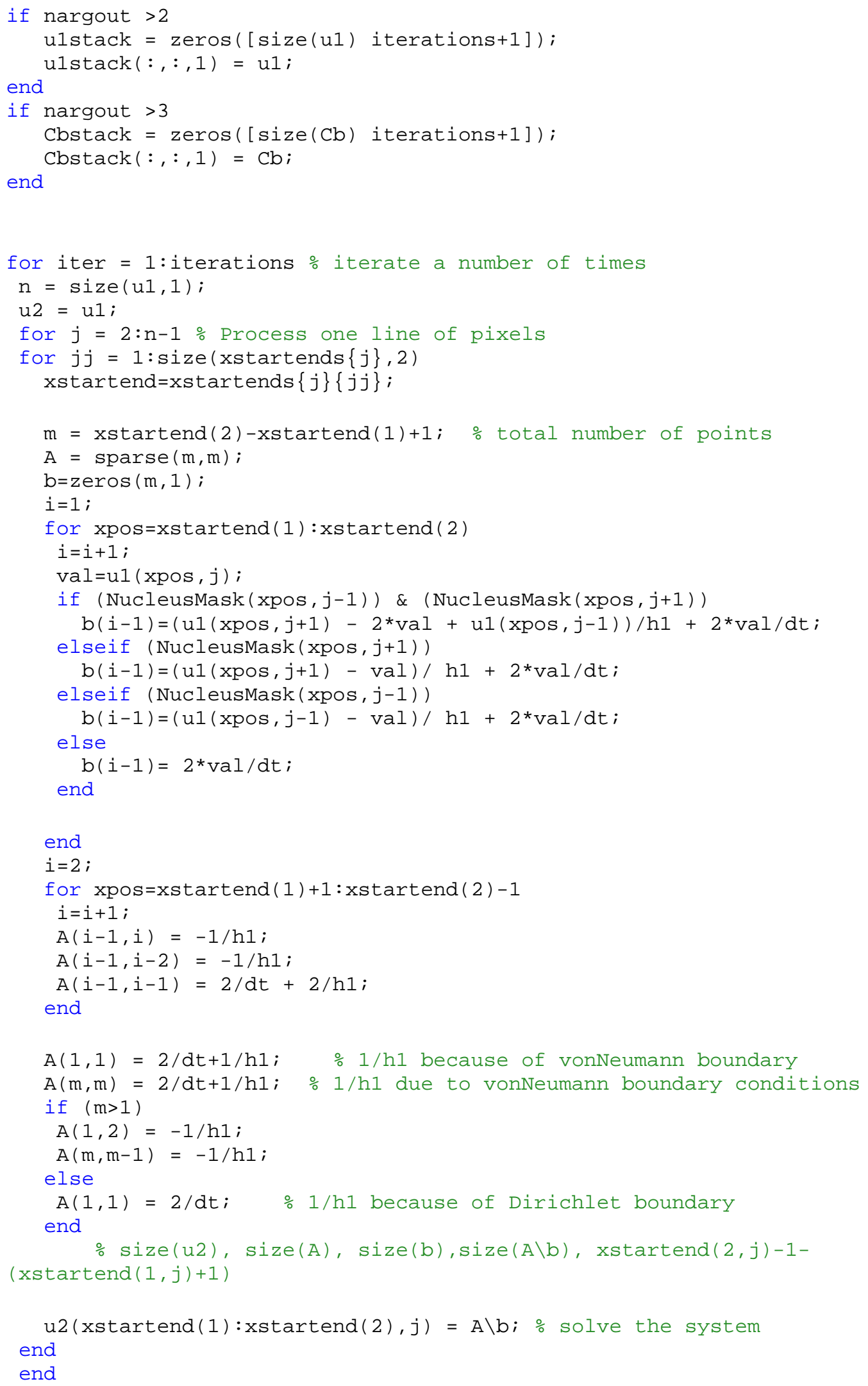




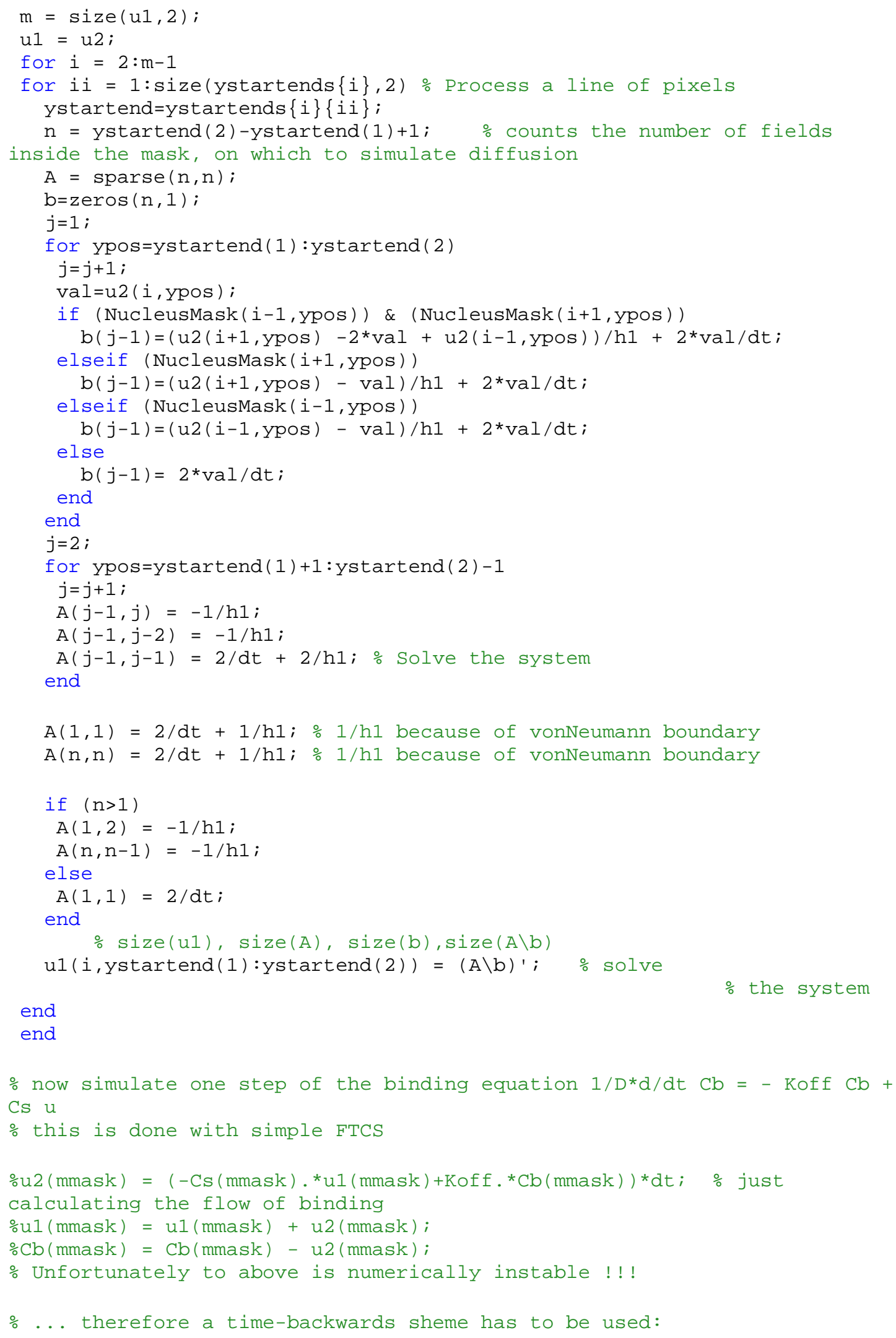




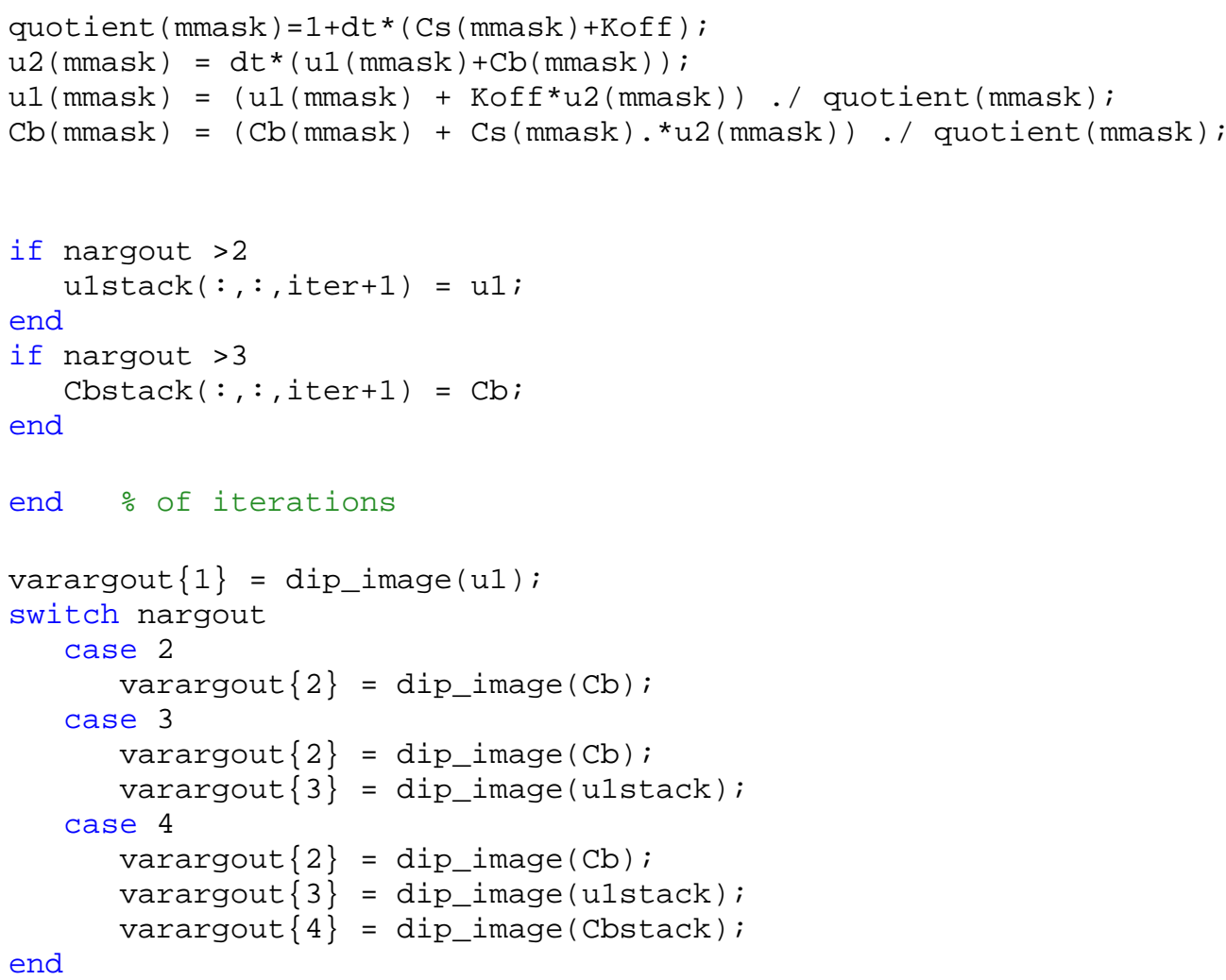

\section{VII.2 Estimation of gamma factors}

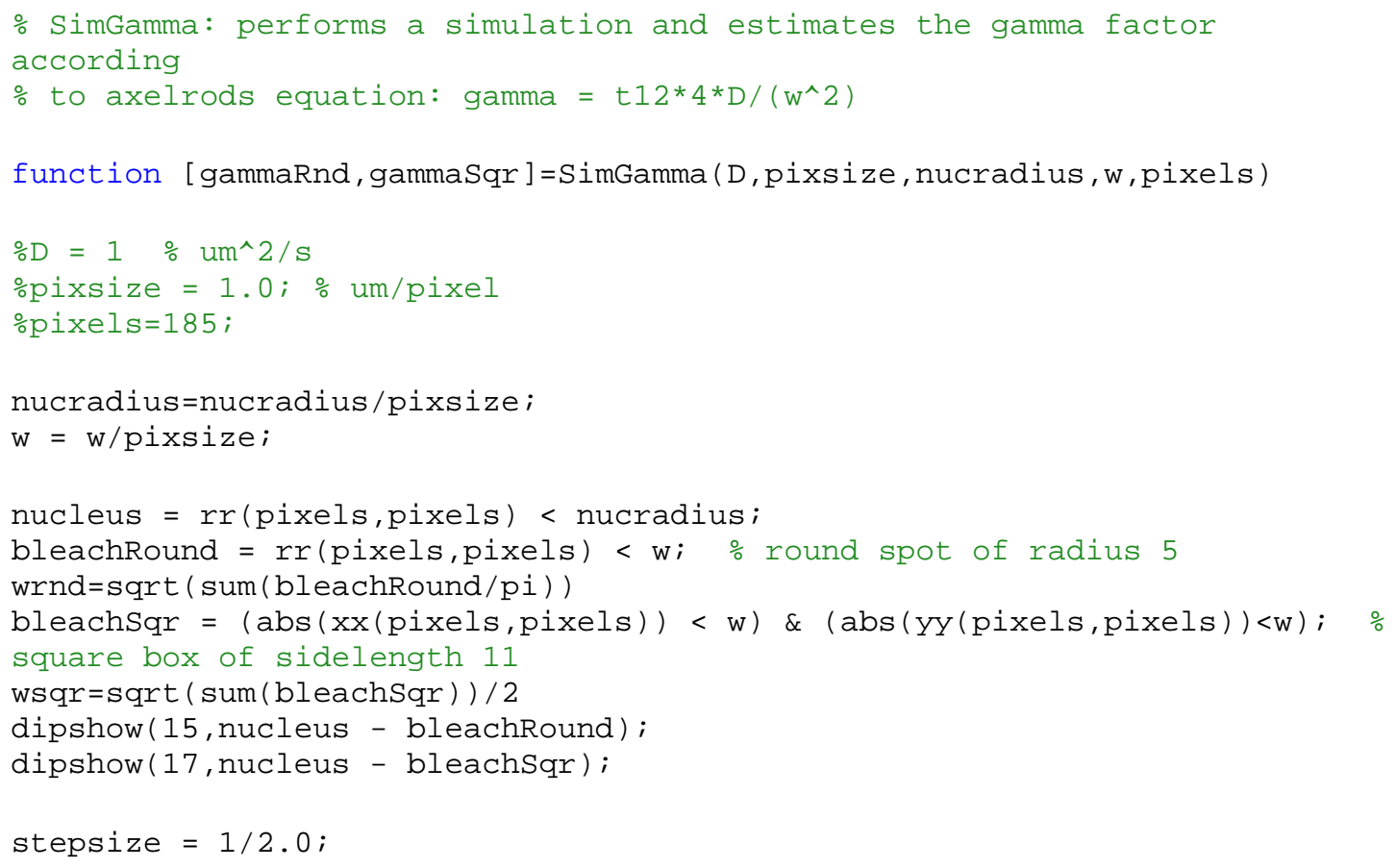




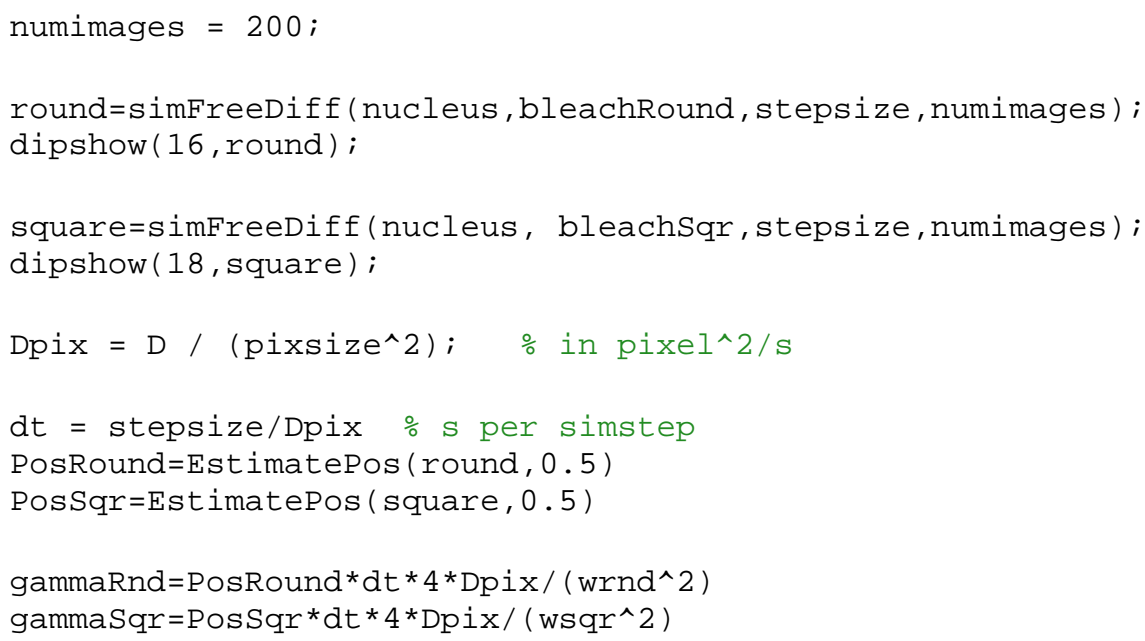

\section{VII.3 Simulation of binding kinetics with given concentrations}

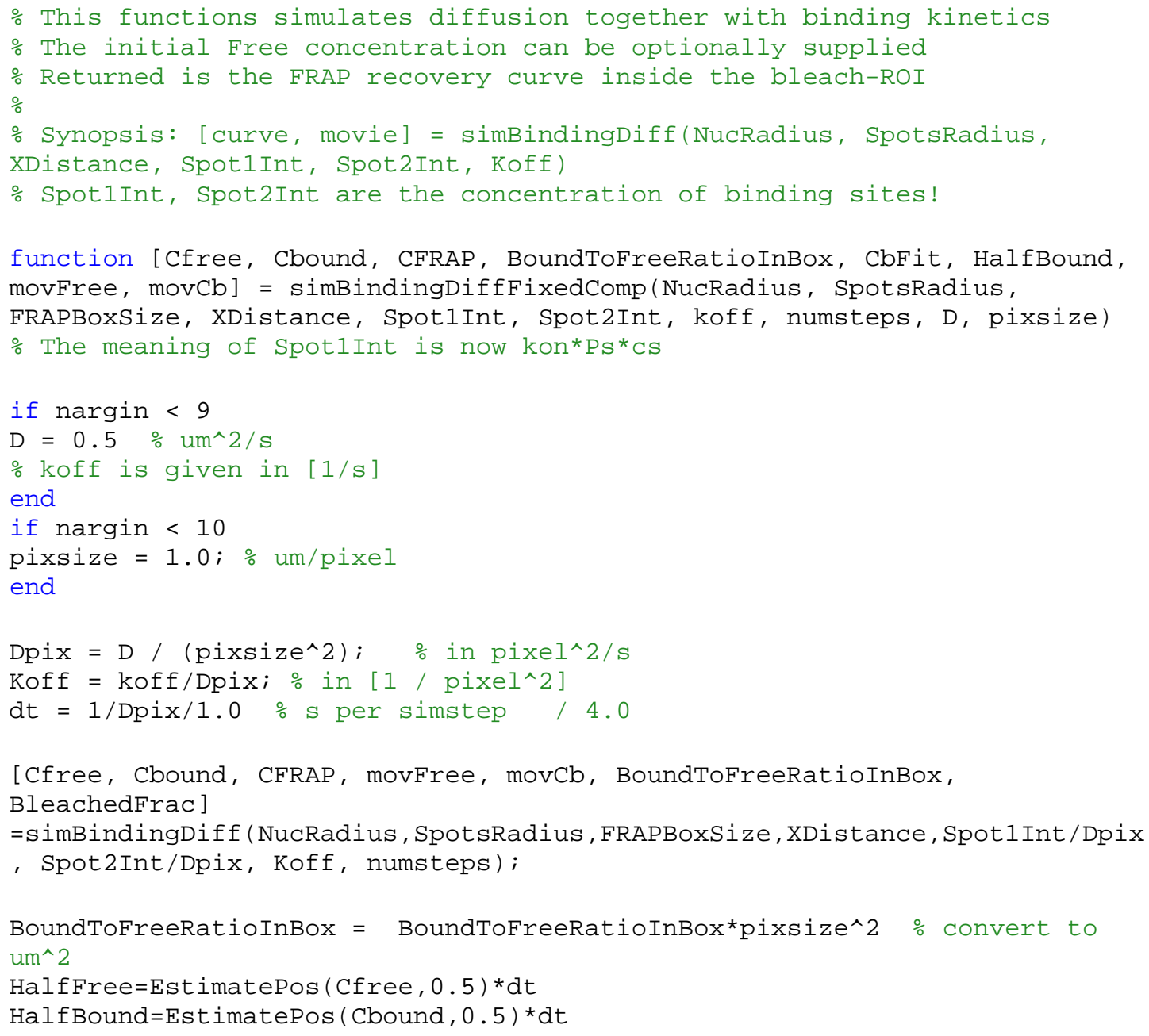




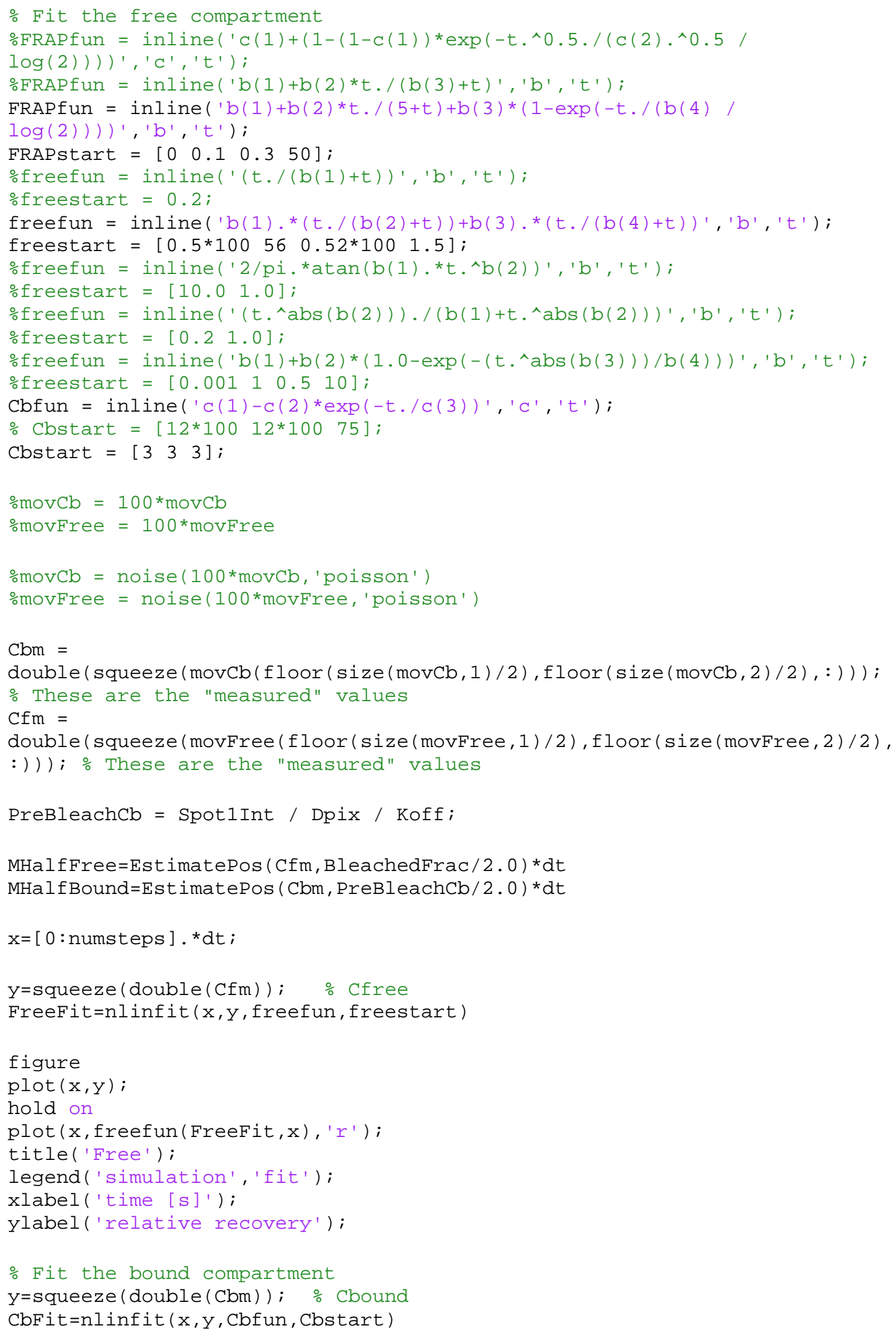




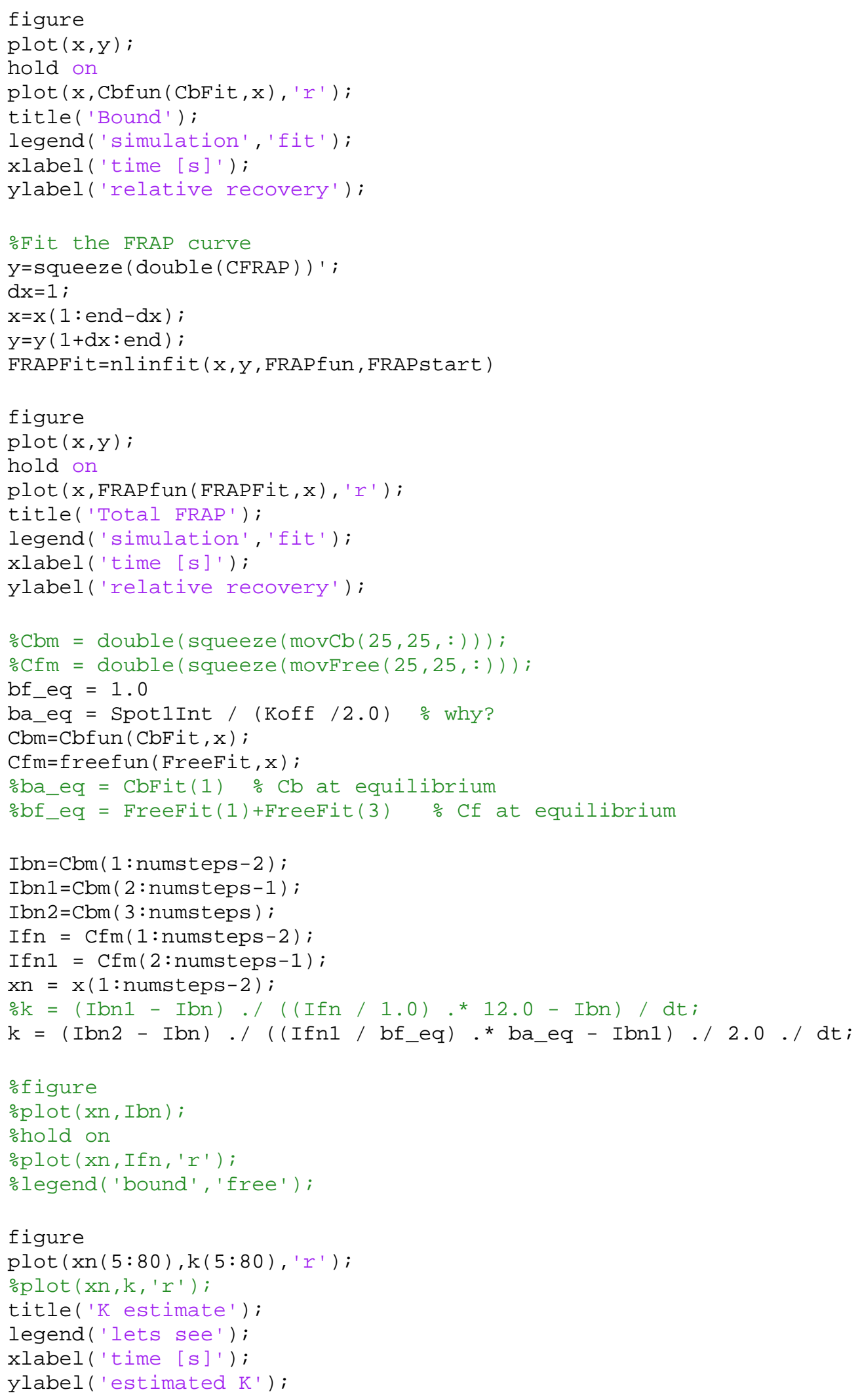




\section{ACKNOWLEDGEMENTS}

My sincere gratitude and thanks go to Dr. Donna Arndt-Jovin for her ongoing support, encouragement and patience during this time. I am thankful for her care, invaluable insight and criticism and for all the discussions we had. My gratitude also goes to Thomas Jovin for giving me the chance to work in his department where I had the possibility to interact with people from so many disciplines.

I also thank the thesis committee members Prof. Herbert Jäckle and Prof. HansJoachim Fritz for discussions and suggestions.

Special thanks are addressed to Greco Hernandez and Cornelia Fritsch for their help and guidance in all the "fly work" and other technical assistance they gave. Thanks for Dr. Peter Schwartz for acquiring electron micrograph pictures and people from Prof. Jäckle’s lab and Dr. Jürg Müller's lab for materials and collaboration. Thanks Bernd Rieger for "brainstorming” for the FRAP kinetic model and simulations.

Of course, all the people in the lab are responsible for the great scientific and social environment that is so important for me. All the evening meetings, game nights, parties, were so great with you. I thank Yvonne Fondufe for her friendship, Wolfgang, Peter and Avishay for their funny jokes and nice characters, Diane for initiating the travels with “Bahnvergnügen” and all the other people, this would be a long list.

I thank the PhD Program in Molecular Biology -International Max Planck Research School at the Georg August University for the great organization and especially Steffen Burckhardt for all he has done for the students and for the success of the program.

I am forever grateful to Rainer Heintzmann for the great scientific experience I had with you, for many hours of discussions, for supporting me in my ups and downs and more than that I am grateful for your love. 


\section{LIST OF FIGURES}

Figure 1 The cell nucleus: organization of the nucleus into specialized domains......- 2 -

Figure 2. Pattern formation in Drosophila.. ................................................................ 5 -

Figure 3. Examples of homeotic mulations. …....................................................................- 6 -

Figure 4. Mechanistic requirements for Polycomb group (PcG) silencing.. ............... 8 -

Figure 5. Interactions among Polycomb group (PcG) proteins.................................- 10 -

Figure 6. Fluorescence recovery after photobleaching (FRAP)............................... 33 -

Figure 7. Inverse FRAP (iFRAP) and three dimensional iFRAP (3D-iFRAP)........- 34 -

Figure 8. Fluorescence Loss In Photobleaching (FLIP)............................................ 35 -

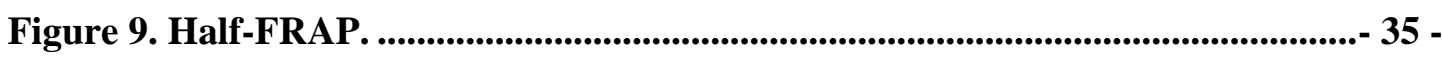

Figure 10. Constructs used to generate transgenic fly lines expressing PcG proteins fused to GFP $44-$

Figure 11. Distribution patterns of PhGFP and PcGFP in the embryos and larval

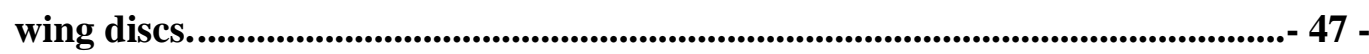

Figure 12. Chromatin dynamics in diploid nuclei ................................................... 50 -

Figure 13. Chromatin movement in various regions of the wing imaginal discs.....- 52 -

Figure 14. Diffusion of PcGFP in nuclei of preblastoderm embryos........................... 54 -

Figure 15. Gamma correction for boundary conditions and box shape................... 57 -

Figure 16. Classical photobleaching techniques in Drosophila embr. and tissues...- 59 -

Figure 17. Secondary bleaching. .......................................................................... 60 -

Figure 18. Simulation of the out-of-focus bleaching................................................- 61 -

Figure 19. Average intensity calculation in a three dimensional weighted volume.- 63 -

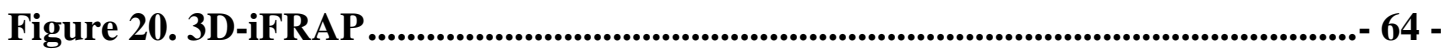

Figure 21. Dissociation rate constants for PhGFP and PcGFP ................................- 66 -

Figure 22. Confocal images of PhGFP and PcGFP expr. salivary gland nuclei ....... 67 -

Figure 23. Kinetic modeling of PcG binding to PREs................................................... 68 -

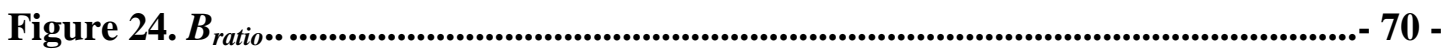

Figure 25. Simulation of the binding kinetics of proteins targeted to localized sites in the nucleus ........................................................................................................................- 72 -

Figure 26 Linear dependence of the recovery time $t_{0}$ on the $B_{\text {ratio }}$............................. 73 - 
Figure 27. Binding processes slow down the FRAP recovery profile for the free diffusion molecules

Figure 28. Distribution PhGFP and PcGFP in the larval salivary gland nuclei and FRAP curves for individual bands. $-77-$

Figure 29. FRAP on the same PcG locus in the salivary gland ................................. 78 Figure 30. Segmentation of the bound fraction and fitting of the FRAP curves .....- 79 -

Figure 31. FRAP on bound PcG proteins in the salivary gland nuclei.......................- 80 Figure 32. Dissociation rate constants for PhGFP and PcGFP and reassociation rate constants for PhGFP 81 -

Figure 33. Polyhomeotic protein binding to chromatin requires the presence of the Polycomb protein ............................................................................................................. 82 -

Figure 34. Effects of PhGFP overexpression in the fly eye and wing ......................... 85 Figure 35. Generation of the GFP-ESC construct.....................................................-8 87 Figure 36. Repeated transient interactions during gene activation ...........................- 88 - 


\section{$X$ LIST OF TABLES}

Table 1 Polycomb group proteins and their function ........................................................ 9 -

Table 2. Primary antibodies .................................................................................... 25 -

Table 3. Quantification of western blots in Fig. 2 G, J and K.....................................- 48 -

Table 4. Diffusion coefficients for PhGFP and PcGFP .............................................. 54 -

Table 5. Parameters for a simulated FRAP .............................................................- 71 -

Table 6. ph null phenotype rescue statistics ................................................................- 83 - 
Statement on the originality of the data

Herewith I declare that I prepared the PhD Thesis "Protein dynamics in the nucleus: Implications for gene expresion" on my own and with no other sources and aids than quoted.

Mathematical modeling of the binding kinetic reactions was done in collaboration with Dr. Rainer Heintzmann and Dr. Bernd Rieger. The simulation programs used in this work have been written by Dr. Rainer Heintzmann. 


\section{Publications}

Ficz .G, Heintzmann, R., Arndt-Jovin D., (2005) "Polycomb group protein complexes exchange rapidly in living Drosophila” Development (accepted)

Ficz .G, Heintzmann, R., Rieger B., Jovin T., Arndt-Jovin D., (2005) “Analysis of binding reactions of localized chromatin binding proteins by Fluorescence Recovery After Photobleaching” (manuscript in preparation)

\section{Master's Thesis}

\section{Gabriella Ficz}

"Comparison of diffusion and dissociation constants for proteins in complexes, which modulate transcription in Drosophila: the Polycomb group repression complex" (International Max-Planck Research School Molecular Biology, 2002)

Oral presentation at the symposium: "Chromatin: Assembly and Inheritance of functional states" in Muenchen, Germany, Oct. 9-11, 2002, entitled: "Mobility of proteins of the Polycomb-Group (Pc-G) and their stability in repression complexes during Drosophila melanogaster development”

Poster presentation at the following meetings:"Dynamic organization of nuclear function” meeting at the Cold Spring Harbor Laboratory, Cold Spring Harbor, New York, Sept. 18- Sept. 22, 2002; FEBS Advanced Workshop: "Nuclear architecture, Chromatin Structure and Gene Control” Wageningen, Netherlands 14-17 November 2003; 46th Symposium of the Society of Histochemistry: “The Cell Nucleus” Prague, Czech Republic 22-25 September 2004; 2nd Alan Wolffe EMBO Conference on Chromatin and Epigenetics, EMBL Heidelberg, Germany, May 19-22, 2005 


\section{Curriculum Vitae}

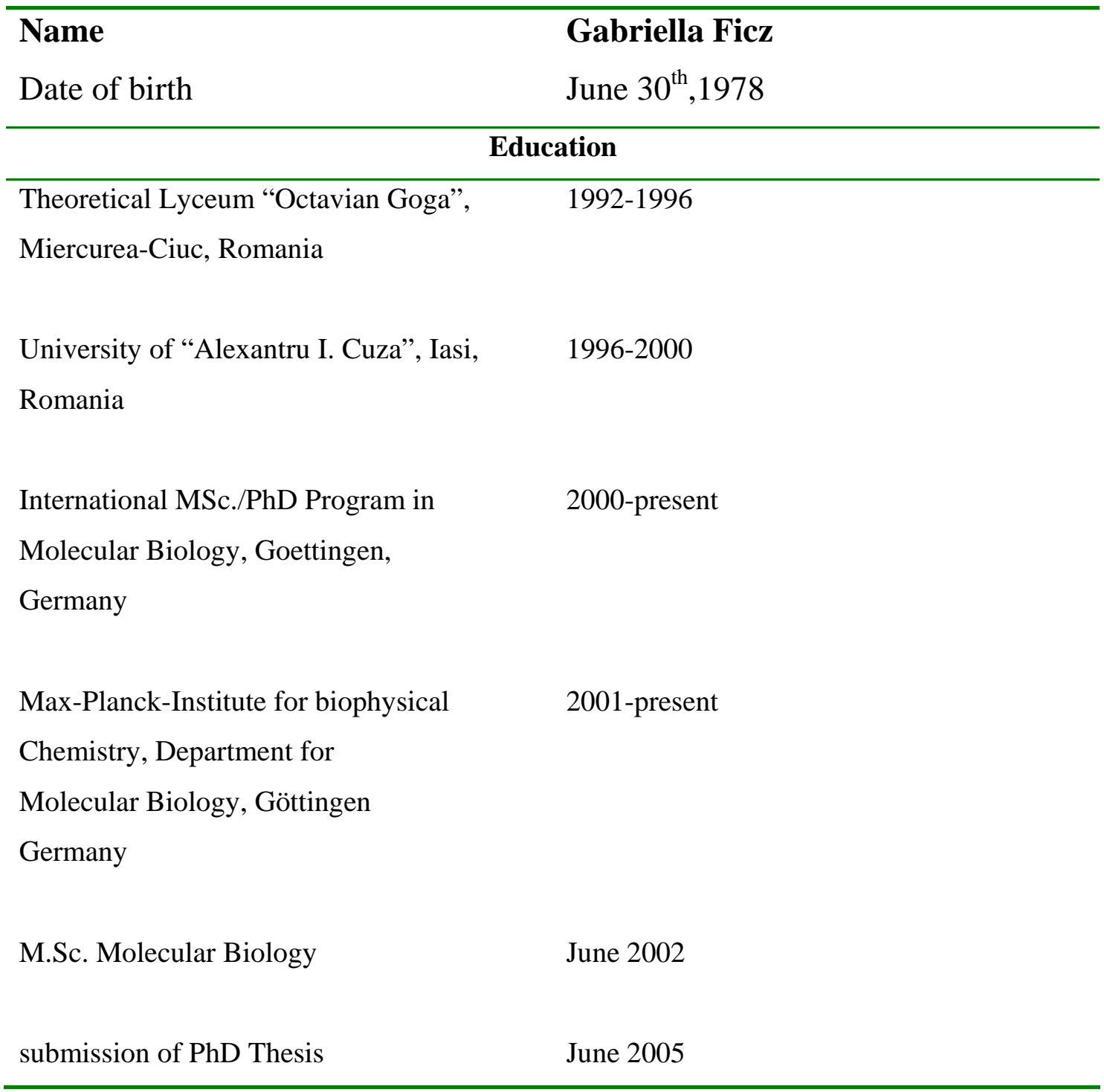

